\title{
Graviton scattering and a sum rule for the c anomaly in 4D CFT
}

\author{
Marc Gillioz, ${ }^{a}$ Xiaochuan Lu ${ }^{b}$ and Markus A. Luty ${ }^{b}$ \\ ${ }^{a}$ Theoretical Particle Physics Laboratory, Institute of Physics, EPFL, \\ CH-1015 Lausanne, Switzerland \\ ${ }^{b}$ Center for Quantum Mathematics and Physics (QMAP), University of California, \\ Davis, California 95616, U.S.A. \\ E-mail: marc.gillioz@epfl.ch, xclu@ucdavis.edu, \\ luty@physics.ucdavis.edu
}

ABSTRACT: 4D CFTs have a scale anomaly characterized by the coefficient $c$, which appears as the coefficient of logarithmic terms in momentum space correlation functions of the energy-momentum tensor. By studying the CFT contribution to 4-point graviton scattering amplitudes in Minkowski space we derive a sum rule for $c$ in terms of TTO OPE coefficients. The sum rule can be thought of as a version of the optical theorem, and its validity depends on the existence of the massless and forward limits of the $\langle T T T T\rangle$ correlation functions that contribute. The finiteness of these limits is checked explicitly for free scalar, fermion, and vector CFTs. The sum rule gives $c$ as a sum of positive terms, and therefore implies a lower bound on $c$ given any lower bound on TTO OPE coefficients. We compute the coefficients to the sum rule for arbitrary operators of spin 0 and 2 , including the energy-momentum tensor.

Keywords: Anomalies in Field and String Theories, Conformal Field Theory

ARXIV EPRINT: 1801.05807 


\section{Contents}

1 Introduction 2

2 Defining the sum rule $\quad 6$

2.1 Completeness relation for CFT states in momentum space 6

2.2 Optical theorem 7

2.3 UV divergences 8

2.4 Contact terms 10

$\begin{array}{lll}2.5 & \text { Polarization tensors } & 12\end{array}$

3 Evaluation of the sum rule $\quad 14$

$\begin{array}{ll}3.1 & \text { Ingredients for the sum rule } \\ \end{array}$

$\begin{array}{ll}3.2 & \text { Conformal blocks from Ward identities } \\ \end{array}$

$\begin{array}{lll}3.3 & \text { Discussion of results } & 18\end{array}$

4 Free theories $\quad 21$

$\begin{array}{lll}4.1 & \text { IR finiteness } & 21\end{array}$

4.2 Scattering rates 22

4.3 Contributing operators 22

4.4 A complete example: the free scalar 23

$\begin{array}{lll}4.5 & T \text { contribution in general CFTs } & 25\end{array}$

5 Conclusions and outlook $\quad 26$

$\begin{array}{lr}\text { A Conventions } & 28\end{array}$

B Computation of the conformal blocks in momentum space 28

B.1 Position space 2-point functions and normalization of operators 29

B.2 Position space 3-point functions and OPE coefficients 29

B.3 Fourier transform of scalar correlators 32

B.4 Conformal Ward identities 32

B.5 Conservation Ward identities 34

B.6 Using Ward identities to compute 3-point functions 35

$\begin{array}{lll}\text { B.7 Relation to position space OPE coefficients } & 39\end{array}$

C IR finiteness for free theories $\quad 41$

$\begin{array}{lll}\text { C.1 Feynman rules } & 42\end{array}$

C.2 IR finiteness of the pseudo-amplitude 43

C.3 Imaginary part of the pseudo-amplitude 46

$\begin{array}{lll}\text { C.4 } \phi^{2} \text { contribution in general } d & 47\end{array}$ 


\section{Introduction}

In this paper we consider two related problems in $4 \mathrm{D}$ conformal field theory (CFT). The first is the computation of physical rates for processes involving particles coupled to a CFT defined by its operator spectrum and operator product expansion (OPE) coefficients. (This has been studied in the phenomenology literature as "unparticle physics" [1,2].) Here we assume that the coupling of the ordinary particles to the CFT is sufficiently weak that it does not affect the dynamics of the CFT. This is similar in spirit to the study of electromagnetism as a probe of QCD, for example in processes like $e^{+} e^{-} \rightarrow$ hadrons or deep inelastic scattering. It is interesting to extend our theoretical understanding of such processes to general CFTs. The second problem we consider is the relation of these rates to scale anomalies in the CFT.

This paper builds on ref. [3], which developed the formalism needed to address these questions for processes involving probe particles without spin. We extend the results to rates involving external gravitons, and relate these to the $c$ anomaly of $4 \mathrm{D} \mathrm{CFT}$. The final result of this work is eq. (1.13), a sum rule that gives $c$ as a positive sum over TTO OPE coefficients, for all primary operators $\mathcal{O}$ other than $\mathbb{1}$ and $T^{\mu \nu}$ itself.

We now give a summary of the ideas that enter into this sum rule. We study the contribution of a 4D CFT to graviton-graviton scattering. This is related to correlation functions of the energy-momentum tensor in the CFT, defined by coupling the CFT to an arbitrary background metric $g_{\mu \nu}$ and differentiating with respect to the metric. For example, the connected 4-point function is given by the time-ordered product

$$
\begin{aligned}
& i^{4}\left\langle 0\left|\mathrm{~T}\left[T^{\mu_{1} \nu_{1}}\left(x_{1}\right) \cdots T^{\mu_{4} \nu_{4}}\left(x_{4}\right)\right]\right| 0\right\rangle_{\mathrm{con}} \\
& \quad=\left.\frac{2}{\sqrt{-g\left(x_{1}\right)}} \cdots \frac{2}{\sqrt{-g\left(x_{4}\right)}} \frac{\delta}{\delta g_{\mu_{1} \nu_{1}}\left(x_{1}\right)} \cdots \frac{\delta}{\delta g_{\mu_{4} \nu_{4}}\left(x_{4}\right)} i W_{\mathrm{CFT}}\left[g_{\mu \nu}\right]\right|_{g_{\mu \nu}=\eta_{\mu \nu}},
\end{aligned}
$$

where $W_{\mathrm{CFT}}\left[g_{\mu \nu}\right]$ is the generating functional of connected correlation functions, the quantum effective action. For example, if the theory has a path integral formulation we have

$$
e^{i W_{\mathrm{CFT}}\left[g_{\mu \nu}\right]}=\int d[\Phi] e^{i S_{\mathrm{CFT}}\left[\Phi, g_{\mu \nu}\right]} .
$$

The probe limit corresponds to the $1 / M_{\text {Planck }}^{4}$ contributions to the graviton-graviton scattering amplitudes. One of the contributions at this order is proportional to the connected momentum-space 4-point function

$$
(2 \pi)^{4} \delta^{4}\left(p_{1}+\cdots+p_{4}\right) i \mathcal{M}_{\lambda_{1} \cdots \lambda_{4}}\left(p_{1}, \ldots, p_{4}\right)=\left\langle 0\left|\mathrm{~T}\left[\tilde{T}_{\lambda_{1}}\left(p_{1}\right) \cdots \tilde{T}_{\lambda_{4}}\left(p_{4}\right)\right]\right| 0\right\rangle_{\mathrm{con}},
$$

where $\lambda_{1}, \ldots, \lambda_{4}$ are the graviton helicities (each equal to \pm 2 ). Here we have taken the Fourier transform and contracted with graviton polarization tensors

$$
\tilde{T}_{\lambda}(p)=\epsilon_{\lambda}^{\mu \nu}(p) \int d^{4} x e^{i p \cdot x} T_{\mu \nu}(x) .
$$

There are additional contributions to the scattering amplitude of order $1 / M_{\text {Planck }}^{4}$ involving intermediate graviton propagators, which are proportional to 2- and 3-point functions of 


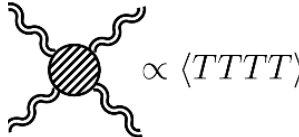

(a)

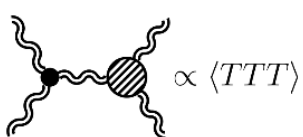

(b)

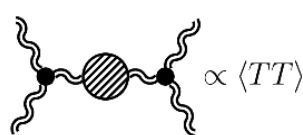

(c)

Figure 1. Contributions to the physical graviton-graviton scattering amplitude at order $1 / M_{\text {Planck }}^{4}$. Curvy double lines denote gravitons, and the blob denotes a correlation function of energymomentum tensors in the CFT. The pseudo-amplitude corresponds to the contribution (a). The contributions (b) and (c) are important for unitarity of the physical graviton amplitude, but are not included in the pseudo-amplitude.

$T^{\mu \nu}$; see figure 1 . The 2- and 3-point functions of the CFT are determined by conformal invariance, so we will focus on eq. (1.3), which we call a "pseudo-amplitude." It is a contribution to a physical graviton scattering amplitude, and it is Lorentz invariant (if the polarization tensors are defined to be Lorentz covariant, as we will discuss below). On the other hand, it does not obey the unitarity relations obeyed by physical graviton scattering amplitudes, such as factorization on graviton poles. However, the CFT is a unitary theory by itself, and eq. (1.3) is a well-defined Lorentz-invariant CFT correlation function. The fact that it is a contribution to a physical graviton amplitude is important for this work only for motivation and physical intuition. We are interested in eq. (1.3) from the point of view of theory rather than phenomenology; that is, we want to see what this quantity can teach us about the CFT itself.

In this paper we will focus on the scale anomaly of the CFT. A scale transformation is equivalent to a Weyl transformation of the form $\delta g_{\mu \nu}=2 \sigma g_{\mu \nu}$ where $\sigma$ is independent of $x$. In a CFT the anomaly in these transformations is by definition local in the effective action:

$$
\delta_{\sigma} W_{\mathrm{CFT}}=c \sigma \int d^{4} x \sqrt{-g} W^{\mu \nu \rho \sigma} W_{\mu \nu \rho \sigma},
$$

where $W_{\mu \nu \rho \sigma}$ is the Weyl tensor. ${ }^{1}$ Eq. (1.5) generates a dilatation of the 4-point function of energy-momentum tensors

$$
\left(\sum_{i=1}^{4} p_{i} \cdot \frac{\partial}{\partial p_{i}}-4\right) \mathcal{M}_{\lambda_{1} \cdots \lambda_{4}}\left(p_{1}, \ldots, p_{4}\right)=c P_{\lambda_{1} \cdots \lambda_{4}}\left(p_{1}, \ldots, p_{4}\right) .
$$

Here $P_{\lambda_{1} \cdots \lambda_{4}}$ is a 4 th order polynomial in the momenta, reflecting the fact that the scale anomaly eq. (1.5) is a local term with 4 derivatives. Eq. (1.6) assumes that the polarization vectors are chosen to be scale invariant, namely

$$
p \cdot \frac{\partial}{\partial p} \epsilon_{\lambda}^{\mu \nu}(p)=0 .
$$

\footnotetext{
${ }^{1}$ The other Weyl anomaly in 4D CFT (the " $a$ anomaly") is proportional to the Euler density (cf. eq. (A.2)), but this is a surface term for $\sigma=$ constant, and therefore does not contribute to the scale anomaly for correlation functions. In theories that have operators with special dimensions (such as marginal operators) there may be additional contributions to the scale anomaly (see e.g. ref. [4, 5]). We will not investigate this possibility in this work.
} 
Naïvely scale invariance (dimensional analysis) implies that the right-hand side of eq. (1.6) should vanish, but $c \neq 0$ in all unitary CFTs, so there is always a nonvanishing scale anomaly. ${ }^{2}$

The scale anomaly is associated with the presence of UV logarithms in the momentum space correlation functions. To get a simple form for these logarithms, we take the massless limit $p_{i}^{2} \rightarrow 0$, and the forward limit $t \rightarrow 0$, where $t$ is the Mandelstam invariant $t=$ $-\left(p_{1}+p_{3}\right)^{2}$ (we use mostly plus metric). With an appropriate Lorentz invariant choice of polarization tensors (see section 2.5 below for details) the forward amplitude is a function only of a single variable, the Mandelstam invariant $s=-\left(p_{1}+p_{2}\right)^{2}$. In this limit we have for example

$$
\mathcal{M}_{++--}(s)=A s^{2}+B s^{2} \ln \frac{-s}{\Lambda^{2}}+B^{\prime} s^{2} \ln \frac{s}{\Lambda^{2}},
$$

for some dimensionless real constants $A, B, B^{\prime}$, and UV cutoff $\Lambda$. (All momenta and helicities are ingoing, so this is a contribution to graviton scattering with helicity structure $++\rightarrow++$.) The pseudo-amplitude is independent of $\Lambda$ because the explicit $\Lambda$ dependence is canceled by the $\Lambda$ dependence of $A$ :

$$
\Lambda \frac{\partial}{\partial \Lambda} A=2\left(B+B^{\prime}\right)
$$

By eq. (1.6) we also have

$$
\left(s \frac{\partial}{\partial s}-2\right) \mathcal{M}_{++--}(s)=\left(B+B^{\prime}\right) s^{2} \propto c s^{2},
$$

where the constant of proportionality is independent of the CFT (and is computed below). We see that the quantities $B$ and $B^{\prime}$ in eq. (1.8) together determine $c$.

We obtain a sum rule for $B$ using the fact that the coefficient of $\ln (-s)$ in eq. (1.8) determines the imaginary part of the pseudo amplitude. By unitarity of the CFT this is related to a positive sum over CFT intermediate states:

$$
\operatorname{Im} \mathcal{M}_{++--}=-B \pi s^{2}=\frac{1}{2} \sum_{|\psi\rangle \neq|0\rangle}\left|\left\langle\psi\left|\mathrm{T}\left[\tilde{T}_{+}\left(p_{1}\right) \tilde{T}_{+}\left(p_{2}\right)\right]\right| 0\right\rangle\right|^{2} .
$$

This can be viewed as a version of the optical theorem. ${ }^{3}$ The vacuum state does not appear in the sum over states in eq. (1.11) because the pseudo-amplitude is a connected correlation function. To obtain a sum rule for $B^{\prime}$, we note that crossing symmetry relates pseudo-amplitudes with different helicity structures. For example, $2 \leftrightarrow 4$ crossing gives

$$
\mathcal{M}_{+--+}(s)=\mathcal{M}_{++--}(-s)=A s^{2}+B s^{2} \ln \frac{s}{\Lambda^{2}}+B^{\prime} s^{2} \ln \frac{-s}{\Lambda^{2}} .
$$

\footnotetext{
${ }^{2}$ Conformal Ward identities imply that the coefficient of the 2-point function of the energy-momentum tensor is proportional to $c$, so $c>0$ in all unitary CFTs.

${ }^{3}$ We emphasize that this "optical theorem" follows from the unitarity of the CFT, and does not depend on the existence of an $S$-matrix for the theory of gravitons coupled to the CFT. (This is discussed further in section 2 below.) For example, we do not need to know if a UV completion of the gravity theory exists.
} 
The imaginary part of this amplitude therefore determines $B^{\prime}$, allowing us to write $c$ as a sum over CFT states.

To compute sums over CFT intermediate states of the form eq. (1.11), we use the stateoperator correspondence in momentum space, which was developed in detail in ref. [3]. This leads to a sum rule of the form

$$
c=\sum_{\substack{\mathcal{O} \subset T \times T \\ \mathcal{O} \neq \mathbb{1}, T}} \sum_{a, b} \lambda_{T T \mathcal{O}}^{(a)} \lambda_{T T \mathcal{O}}^{(b)} f_{a b}(\mathcal{O})
$$

where the first sum is over all primary operators $\mathcal{O}$ appearing in the TT OPE. The identity operator does not appear in the sum because it corresponds to the contribution of the vacuum state in eq. (1.11). The energy-momentum tensor does not appear because we will show that its contribution is itself proportional to $c$, and has been absorbed into the lefthand side. The sum over $a$ and $b$ runs over the different kinematic structures in the TTO 3 -point function, $\lambda_{T T \mathcal{O}}^{(a)}$ are the corresponding OPE coefficients, and $f_{a b}(\mathcal{O})$ is a positivedefinite matrix that depends only on the quantum numbers of $\mathcal{O}$. The coefficients $f_{a b}(\mathcal{O})$ are kinematic in the sense that they are completely determined by conformal invariance. We will give explicit formulas for these coefficients in the case where $\mathcal{O}$ has spin 0 or 2 . Because eq. (1.13) is a sum of positive terms, any finite number of terms gives a lower bound on $c$, potentially a very useful constraint in the conformal bootstrap program.

There are a number of technical points that must be understood before claiming the validity of the sum rule eq. (1.13). Correlation functions of the energy-momentum tensors in momentum space can have UV divergences and contact terms that are not fixed by symmetries. In addition, the polarization tensors must depend on reference momenta in order to be Lorentz invariant. We will discuss these points in detail and show that the sum rule does not depend on them. On the other hand, we do not have a complete understanding of IR divergences, specifically possible divergences in the massless and forward limits $p_{i}^{2} \rightarrow$ $0, t \rightarrow 0$ described above. We have checked that for free CFTs (free scalars, fermions, and vectors) there are no IR divergences in this limit, and the sum rule is valid for these theories. The imaginary part of pseudo-amplitudes that enters into the optical theorem is a total rate, which is expected to be an IR safe observable for interacting theories. However, IR divergences in the real part could also invalidate our sum rule. For example a crossing symmetric contribution of the form

$$
\mathcal{M}_{++--}(s, t, u) \sim\left(s^{2}+t^{2}+u^{2}\right)\left[\ln \frac{s}{\Lambda^{2}}+\ln \frac{t}{\Lambda^{2}}+\ln \frac{u}{\Lambda^{2}}\right]
$$

would give a finite contribution to the imaginary part in the forward limit $t \rightarrow 0$. This contribution does not have the form eq. (1.8) in the forward limit, and therefore invalidates the sum rule. We note that the relationship between forward amplitudes and scale anomalies was pioneered in the original proof of the $a$-theorem $[6]^{4}$ and used in non-perturbative arguments that scale implies conformal symmetry $[8,9]$. These works also rely on the

\footnotetext{
${ }^{4}$ There is a completely independent proof for the $a$-theorem using properties of entanglement entropy that does not require any such assumption [7].
} 
assumption that a similar forward amplitude involving the trace of the energy-momentum tensor is free of IR divergences. The validity of the sum rule for free theories is very encouraging, but a better understanding of IR divergences would be very reassuring. We leave this for future work.

The sum rule eq. (1.13) is similar to a bound on the TTT OPE coefficient $n_{\mathrm{s}}$ that was recently derived in [10]. This lower bound is given by a positive sum of TTO OPE coefficients squared, where $\mathcal{O}$ is a scalar primary operator. The bound is based on the average null energy condition, which has been recently proven using several different methods $[11,12]$. The bound can also be obtained from requiring unitarity of correlation functions in the Regge limit [13]. It would be interesting to understand the connection between our results and this bound.

This paper is organized as follows. In section 2 we give precise definitions and additional details about the quantities that enter into the sum rule: we define the sum over CFT states, derive the optical theorem, discuss UV divergences and contact terms, and define the polarization tensors. In section 3 we carry out the computations needed to evaluate the contributions of operators with spin 0 and 2 to the sum rule, including the energy-momentum tensor. The final form of our sum rule is given there. In section 4 we study free field CFTs. We show that they are free from IR divergences, use them to compute the contribution of states created by the energy-momentum tensor, and check the convergence of the sum rule. In section 5 we give our conclusions and outlook.

The reader interested in getting an overview of our results is encouraged to skip to section 3 , referring to section 2 and the appendices as needed to fill in missing details.

\section{Defining the sum rule}

\subsection{Completeness relation for CFT states in momentum space}

The main technical tool that enables our results is the Minkowski momentum space completeness relation $[3]^{5}$

$$
\mathbb{1}=\sum_{\mathcal{O}} \int \frac{d^{4} k}{(2 \pi)^{4}} \theta\left(-k^{2}\right) \theta\left(k^{0}\right)\left[\Pi_{\mathcal{O}}^{-1}(k)\right]_{\mu_{1} \ldots \mu_{\ell}, \nu_{1} \ldots \nu_{\ell}} \tilde{\mathcal{O}}^{\mu_{1} \ldots \mu_{\ell}}(k)|0\rangle\langle 0| \tilde{\mathcal{O}}^{\nu_{1} \ldots \nu_{\ell}}(-k)
$$

The $\theta$ functions enforce the condition that physical states have positive energy and timelike momentum $\left(k^{2}<0\right)$. The sum is over all primary operators $\mathcal{O}$, and $\Pi_{\mathcal{O}}^{-1}(k)$ is the inverse of the tensor appearing in the Wightman 2-point function:

$$
\left\langle 0\left|\tilde{O}^{\mu_{1} \cdots \mu_{\ell}}\left(-k^{\prime}\right) \tilde{O}^{\nu_{1} \cdots \nu_{\ell}}(k)\right| 0\right\rangle=(2 \pi)^{4} \delta^{4}\left(k^{\prime}-k\right) \theta\left(-k^{2}\right) \theta\left(k^{0}\right)\left[\Pi_{\mathcal{O}}(k)\right]^{\mu_{1} \ldots \mu_{\ell}, \nu_{1} \ldots \nu} .
$$

\footnotetext{
${ }^{5}$ In ref. [3] the completeness relation is written with the operators inserted at a finite imaginary time in Minkowski space. The completeness relation is independent of this imaginary time, and here we take the limit where the imaginary time goes to zero, which leaves the usual $i \epsilon$ prescription defining the Wightman ordering of the insertion of the operators.
} 
The tensor $\Pi_{\mathcal{O}}^{-1}(k)$ can be written as a sum over suitably normalized polarization tensors of the form ${ }^{6}$

$$
\left[\Pi_{\mathcal{O}}^{-1}(k)\right]_{\mu_{1} \ldots \mu_{\ell}, \nu_{1} \ldots \nu_{\ell}}=\sum_{\lambda}\left[\epsilon_{\mu_{1} \ldots \mu_{\ell}}^{\lambda}(k)\right]^{*} \epsilon_{\nu_{1} \ldots \nu_{\ell}}^{\lambda}(k)
$$

so that the completeness relation can be written in a more compact form

$$
\mathbb{1}=\sum_{\mathcal{O}} \sum_{\lambda} \int \frac{d^{4} k}{(2 \pi)^{4}} \theta\left(-k^{2}\right) \theta\left(k^{0}\right) \tilde{\mathcal{O}}_{\lambda}(k)|0\rangle\langle 0| \tilde{\mathcal{O}}_{\lambda}(-k),
$$

where $\mathcal{O}_{\lambda}=\epsilon_{\mu_{1} \cdots \mu_{\ell}}^{\lambda} \mathcal{O}^{\mu_{1} \cdots \mu_{\ell}}$.

Eq. (2.4) is simple but quite nontrivial. A detailed derivation is given in ref. [3]. (See also ref. [14].) It follows from the operator-state correspondence, together with the fact that the Fourier transform of a primary operator contains the correct contribution of the descendants. To understand the latter point, note that the Fourier transform can be written

$$
\tilde{\mathcal{O}}(k)=\int d^{4} x e^{i k \cdot x} e^{x^{\mu} \partial_{\mu}} \mathcal{O}(0)
$$

This shows that $\tilde{\mathcal{O}}(k)$ is the unique linear combination of descendants of $\mathcal{O}(0)$ with momentum $k$. Note that as an operator relation, eq. (2.4) can only be inserted between operators with a fixed (Wightman) ordering. For such correlation functions, eq. (2.4) says that the conformal block is essentially given by the product of the Fourier transform of the 3-point functions with the primary operator being inserted.

In this paper we apply the completeness relation to the sum over states on the righthand side of eq. (1.11), which will be derived in the following subsection. This gives

$$
\sum_{|\psi\rangle \neq|0\rangle}\left|\left\langle\psi\left|\mathrm{T}\left[\tilde{T}_{+}\left(p_{1}\right) \tilde{T}_{+}\left(p_{2}\right)\right]\right| 0\right\rangle\right|^{2}=\sum_{\mathcal{O}} \sum_{\lambda}\left|\mathcal{M}_{T_{1} T_{2} \rightarrow \mathcal{O}_{\lambda}}\left(p_{1}, p_{2}\right)\right|^{2},
$$

where we use the notation

$$
(2 \pi)^{4} \delta^{4}\left(p_{1}+p_{2}-k\right) \mathcal{M}_{T_{1} T_{2} \rightarrow \mathcal{O}_{\lambda}}\left(p_{1}, p_{2}\right)=\left\langle 0\left|\tilde{\mathcal{O}}_{\lambda}(-k) \mathrm{T}\left[\tilde{T}_{1}\left(p_{1}\right) \tilde{T}_{2}\left(p_{2}\right)\right]\right| 0\right\rangle .
$$

for the 3-point functions. As the notation suggests, $\mathcal{M}_{T_{i} T_{j} \rightarrow \mathcal{O}_{\lambda}}$ is itself a pseudo-amplitude describing the inverse decay of two massless gravitons to a CFT state with mass $m^{2}=s$.

\subsection{Optical theorem}

We now turn to the "optical theorem" eq. (1.11). This follows from the combinatoric identity

$$
\sum_{k=0}^{n}(-1)^{k} \sum_{\sigma \in \Pi(k, n-k)} \overline{\mathrm{T}}\left[\mathcal{O}\left(x_{\sigma_{1}}\right) \cdots \mathcal{O}\left(x_{\sigma_{k}}\right)\right] \mathrm{T}\left[\mathcal{O}\left(x_{\sigma_{k+1}}\right) \cdots \mathcal{O}\left(x_{\sigma_{n}}\right)\right]=0,
$$

\footnotetext{
${ }^{6}$ This follows from the Källén-Lehmann representation for the Wightman 2-point function

$$
\Pi^{\mu_{1} \cdots \mu_{\ell}, \nu_{1} \cdots \nu_{\ell}}(k)=\theta\left(k^{0}\right) \theta\left(-k^{2}\right) \sum_{\lambda} \rho_{\lambda}\left(k^{2}\right)\left[\hat{\epsilon}_{\lambda}^{\mu_{1} \ldots \mu_{\ell}}(k)\right]^{{ }^{*}} \hat{\epsilon}_{\lambda}^{\nu_{1} \cdots \nu_{\ell}}(k)
$$

where the hatted polarization tensors are orthonormal: $\left(\hat{\epsilon}_{\lambda^{\prime}}\right)^{*} \cdot \hat{\epsilon}_{\lambda}=\delta_{\lambda^{\prime} \lambda}$. The density of states $\rho_{\lambda}\left(k^{2}\right)$ is positive-definite, so we can invert the 2-point function to obtain eq. (2.2).
} 
where sum runs over all partitions $\sigma$ of $1, \ldots, n$ into two groups of size $k$ and $n-k$. This identity is proved by writing out all the (anti-)time orderings and checking that they cancel pairwise. We apply this to the 4-point function eq. (1.3) Fourier-transformed to momentum space, with the kinematics

$$
p_{i}^{2}=0, \quad s=-\left(p_{1}+p_{2}\right)^{2}>0, \quad t=-\left(p_{1}+p_{3}\right)^{2} \leq 0 .
$$

This corresponds to a physical $2 \rightarrow 2$ scattering process with incoming momenta $p_{1}$ and $p_{2}$ and outgoing momenta $-p_{3}$ and $-p_{4}$. With this choice, most of the terms in eq. (2.8) do not contribute because the momentum flow between the time-ordered and anti timeordered products is unphysical. The only partition that survives is the $s$-channel, and we obtain

$$
(2 \pi)^{4} \delta^{4}\left(p_{1}+\ldots+p_{4}\right) \operatorname{Im} \mathcal{M}(s, t)=\frac{1}{2}\left\langle 0\left|\overline{\mathrm{T}}\left[\tilde{T}_{4}\left(p_{4}\right) \tilde{T}_{3}\left(p_{3}\right)\right] \mathrm{T}\left[\tilde{T}_{2}\left(p_{2}\right) \tilde{T}_{1}\left(p_{1}\right)\right]\right| 0\right\rangle .
$$

The completeness relation (2.1) can now be inserted on the right-hand side of this equality to rewrite it as a sum of products of 3-point functions. We then take the forward limit $p_{3} \rightarrow-p_{1}, p_{4} \rightarrow-p_{2}(t \rightarrow 0)$, and also choose the polarizations of $\tilde{T}_{3}$ and $\tilde{T}_{4}$ to be respectively the same as $\tilde{T}_{1}$ and $\tilde{T}_{2}$, so that we have forward kinematics. The right-hand side of eq. (2.10) becomes a sum of squares, which gives eq. (2.6) above.

\section{$2.3 \quad$ UV divergences}

We now consider UV divergences in momentum space CFT correlation functions. The CFT correlation functions in position space at finite separation are unambiguously determined by the CFT data. However, the Fourier transform to momentum space involves the integration of the position space correlation function over coincident points, potentially introducing ambiguities from UV divergences and contact terms. We will discuss UV divergences in this subsection. Contact terms are discussed in the following subsection.

UV divergences are in one-to-one correspondence with counterterms that are generally covariant, local, and involve relevant or marginal operators. In $4 \mathrm{D}$, the possible UV divergent counterterms for the 4-graviton pseudo-amplitude are $^{7}$

$$
\Delta S \sim \int d^{4} x \sqrt{-g}\left[\Lambda^{4}+\Lambda^{2} R+\ln \Lambda R^{2}+\ln \Lambda W^{\mu \nu \rho \sigma} W_{\mu \nu \rho \sigma}\right],
$$

where $\Lambda$ is a UV cutoff scale. However, because these counterterms are local they do not contribute to the imaginary part of the pseudo-amplitude, and therefore do not invalidate our sum rule. ${ }^{8}$ For example, in eq. (1.8) these divergences would contribute to the coefficient $A$, which is not important for us.

More dangerous are UV divergences that can contribute to the 3-point functions on the right-hand side of the optical theorem eq. (2.6). These involve Fourier transforms of

\footnotetext{
${ }^{7}$ There are only two curvature-squared counterterms because the Euler term $\sqrt{-g} E_{4}$ is a total derivative.

${ }^{8}$ In fact, the power divergences can be tuned away, and the $R^{2}$ term breaks conformal invariance, and is therefore absent by assumption. The log-divergent $W^{2}$ term is associated with the $c$ anomaly that is the subject of this paper.
} 
3-point functions of the form

$$
\left\langle 0\left|\mathcal{O}^{\alpha_{1} \cdots \alpha_{\ell}}(z) \mathrm{T}\left[T^{\mu \nu}(x) T^{\rho \sigma}(y)\right]\right| 0\right\rangle .
$$

In the Fourier transform of this quantity, there are no UV divergences arising from the region where $z \rightarrow x$ or $z \rightarrow y$. Physically this is because the integral over $z$ is computing the sum over final states with energy and momentum fixed by the total momentum in the initial state created by the time-ordered product, and such sums over states cannot have UV divergences because the 4-momentum of the final state is fixed. More formally, this can be understood from the results of ref. [3], which showed that we can compute the Fourier transform over $z$ in eq. (2.12) with $z^{0}$ having a finite nonzero imaginary part. The dependence on the imaginary part exactly cancels with the normalization of the state, and so Fourier transform can be computed without integrating over points where $z=x$ or $y$.

The only UV divergences in the Fourier transform of eq. (2.12) therefore arise from the coincident limit $x \rightarrow y$. These UV divergences are in one-to-one correspondence with relevant or marginal counterterms coupling the metric to $\mathcal{O}$. In $4 \mathrm{D}$, the only generally covariant relevant or marginal counterterms are

$$
\Delta S \sim \int d^{4} x \sqrt{-g}\left[\Lambda^{4-\Delta_{1}} \mathcal{O}_{1}+\Lambda^{2-\Delta_{2}} R \mathcal{O}_{2}\right]
$$

where $\mathcal{O}_{1,2}$ are primary scalar operators with dimensions $\Delta_{1} \leq 4, \Delta_{2} \leq 2$. (For $\Delta_{1}=4$ or $\Delta_{2}=2$ the powers of $\Lambda$ become logarithms.) Counterterms involving higher spin operators or descendants are forbidden by unitarity constraints on the dimensions, together with the fact that we can neglect total derivative terms. In addition the operators $\mathcal{O}_{1,2}$ must be singlets under all global symmetries of the CFT. For example, we need not consider the operator $\bar{\psi} \psi$ in free fermion theory because it is not a singlet under chiral symmetry. We also need not consider the identity operator, since it does not appear in the sum over states eq. (1.13).

We begin with the counterterm $\mathcal{O}_{1}$ in eq. (2.13). Note that this is a perturbation of the CFT dynamics even in flat spacetime, and therefore represents a genuine breaking of conformal invariance. Even if $\mathcal{O}_{1}$ is an exactly marginal operator, the presence of logarithmic divergences proportional to $\mathcal{O}_{1}$ breaks conformal symmetry. Because $\mathcal{O}_{1}$ has non-local correlation functions, such terms will give rise to non-local terms in the quantum effective action that break conformal invariance. Such terms are therefore absent by our assumption of conformal invariance. For example, if $\mathcal{O}_{1}$ is relevant $\left(\Delta_{1}<4\right)$ then such UV divergent terms must be tuned away to get conformal invariance.

We next consider the counterterm $R \mathcal{O}_{2}$ in eq. (2.13). It does not affect the dynamics of the CFT in flat spacetime, but it does change the definition of the energy-momentum tensor, which follows from differentiating with respect to the metric. The term $R \mathrm{O}_{2}$ is not Weyl invariant, and therefore leads to a breaking of conformal invariance in correlation functions involving the energy-momentum tensor. (Effectively, it mixes $T^{\mu \nu}$ with the operator $\partial^{\mu} \partial^{\nu} \mathcal{O}$, which violates conformal invariance because the latter operator is a descendant.) The situation is therefore quite similar to the counterterms involving $\mathcal{O}_{1}$, namely such UV divergences must be absent by the assumption of conformal invariance. For example, in 
a free scalar conformal field theory the term $\phi^{2} R$ does not have a logarithmic divergence, consistent with the fact that this theory is conformally invariant.

\subsection{Contact terms}

We now classify the possible contact terms that can occur in the CFT correlation functions that appear in this work, and explain why contact term ambiguities do not affect the sum rule. Although they cancel in the final results, contact terms unavoidably appear in intermediate steps, and it is essential to include them correctly.

Contact terms are contributions to operator products that are localized at coincident points, for example

$$
\mathrm{T}\left[T^{\mu \nu}(x) T^{\rho \sigma}(y)\right]=\delta^{4}(x-y) \mathcal{O}^{\mu \nu \rho \sigma}(x)+\cdots,
$$

where we absorb a possible coefficient into the normalization of the operator $\mathcal{O}$. We can ignore contact terms in position space simply by staying away from coincident points, but the Fourier transform to momentum space includes integration over all points, and contact terms cannot be ignored.

We first claim that the only contact terms that can appear in correlation functions are between operators within a time ordering. This can be understood from the fact that both Wightman and time-ordered products are defined by analytic continuation from Euclidean correlation functions, which may have contact terms at coincident points. Wightman products are defined using an $i \epsilon$ prescription where operators to the left have a larger negative imaginary time. This prevents the times of Wightman-ordered operators from coinciding, and therefore there are no contact terms between such operators. ${ }^{9}$ On the other hand, time-ordered products are defined by a common Wick rotation, which allows the location of the analytically continued operators to coincide. This means that we only need to consider contact terms between energy-momentum tensors in this work, such as eq. (2.14) above. There are also possible contact terms where 3 or more energy-momentum tensors coincide. Such contributions do not appear in the imaginary part of the pseudo-amplitude $\mathcal{M}$, and we do not need to consider them.

Conformal invariance requires that the operator $\mathcal{O}$ appearing in the contact term eq. (2.14) has dimension 4. One operator that can always appear in such contact terms is the energy-momentum tensor itself. As we now explain, these contact terms are completely determined by the definition eq. (1.1) of energy-momentum tensor correlation functions. Eq. (1.1) is equivalent to writing the metric as

$$
g_{\mu \nu}(x)=\eta_{\mu \nu}+h_{\mu \nu}(x)
$$

and defining $T^{\mu \nu}$ by differentiating with respect to the source $h_{\mu \nu}$. Equivalently, the $T^{\mu \nu}$ correlation functions are the coefficients of the expansion of the quantum effective action

\footnotetext{
${ }^{9}$ This is essentially the same argument used above to show that UV divergences do not occur when Wightman-ordered products approach each other.
} 
in powers of $h_{\mu \nu}$ :

$$
\begin{aligned}
i W_{\mathrm{CFT}}\left[g_{\mu \nu}\right]= & \sum_{n=1}^{\infty} \frac{1}{n !} \int d^{4} x_{1} \cdots d^{4} x_{n} h_{\mu_{1} \nu_{1}}\left(x_{1}\right) \cdots h_{\mu_{n} \nu_{n}}\left(x_{n}\right) \\
& \times\left(\frac{i}{2}\right)^{n}\left\langle 0\left|\mathrm{~T}\left[T^{\mu_{1} \nu_{1}}\left(x_{1}\right) \cdots T^{\mu_{n} \nu_{n}}\left(x_{n}\right)\right]\right| 0\right\rangle_{\mathrm{con}}
\end{aligned}
$$

That is, $n$-point functions of $T^{\mu \nu}$ are the "interaction terms" with $n$ powers of $h_{\mu \nu}$ in the quantum effective action. However, one could have instead made a different definition of the energy momentum tensor, namely by taking

$$
h_{\mu \nu}^{\prime}=h_{\mu \nu}+\alpha_{1} h_{\mu}^{\rho} h_{\rho \nu}+\alpha_{2} h h_{\mu \nu}+\alpha_{3} h^{2} \eta_{\mu \nu}+O\left(h^{3}\right),
$$

where $\alpha_{1}, \alpha_{2}, \ldots$ are arbitrary constants, and we raise and lower indices with the flat metric in this expression. We can then define a new energy-momentum tensor $T^{\prime \mu \nu}$ as the coefficient in the expansion in $h_{\mu \nu}^{\prime}$, similar to eq. (2.16). By writing $h_{\mu \nu}^{\prime}$ in terms of $h_{\mu \nu}$, we see that correlation functions of the different energy-momentum tensors agree except at coincident points. This is the contact term ambiguity for operators $\mathcal{O}$ in eq. (2.14) that involve the energy-momentum tensor.

Eq. (2.17) can be thought of as a redefinition of the graviton field, and physical (onshell) graviton amplitudes are invariant under such field redefinitions. On the other hand, our pseudo-amplitude is not invariant under these redefinitions. However, this ambiguity cancels on both sides of our sum rule and therefore do not affect the final result, as we now explain.

In the pseudo-amplitude $\mathcal{M}\left(p_{1}, \ldots, p_{4}\right)$ defined in eq. (1.3) we need only worry about contact terms between 1 and 2, or between 3 and 4 . Contact terms between other pairs of momenta will have a vanishing imaginary part, and therefore do not contribute to the optical theorem. Contact terms between 1 and 2 do change the value of the amplitude, but they also change the the right-hand side of the optical theorem, and the difference cancels. This is because the contact terms such as eq. (2.14) can be viewed as an operator statement that appears on both sides of the sum rule. Since the sum rule is the insertion of a complete set of states, the addition of the same operator on both sides of the sum rule does not affect the result. In fact, on the right-hand side of the sum rule the contact terms only affect the contribution from the states $\tilde{T}^{\mu \nu}(p)|0\rangle$, since states created by the energy-momentum tensor are orthogonal to states created by other primary operators. Once the contribution of the energy-momentum tensor is included, the sum rule has no contact term ambiguities term by term.

Although the final result is independent of contact terms, we must be careful to compute both sides of the sum rule with the same contact terms. For free field CFTs, this is a matter of using the Feynman rules for the fields $h_{\mu \nu}$ defined in eq. (2.15). For general CFTs, we will see that the contact terms that enter on the right-hand side of the sum rule are completely fixed by using the Ward identities for conservation and conformal invariance, which encode the definition eq. (1.1) as well as the ordering (time-ordered or Wightman) of the correlation functions. In a number of cases we have used both methods and checked that they agree. This will be discussed in more detail below. 
We next consider contact terms that involve operators $\mathcal{O}$ on the right-hand side of eq. (2.14) that are not equal to the energy-momentum tensor. By conformal invariance, these can only occur when there are operators with special dimensions, and therefore do not appear in generic CFTs. Such contact terms are associated with terms in the action that couple the operator $\mathcal{O}$ to the source for $T^{\mu \nu}$, namely the metric. The only such term compatible with conformal invariance is

$$
\Delta S=\int d^{4} x \sqrt{-g} \rho \mathcal{O}
$$

where $\mathcal{O}$ is an exactly marginal operator, and $\rho$ is an arbitrary coupling. ${ }^{10}$ This gives rise to contact terms of the form

$$
\mathrm{T}\left[T^{\mu \nu}(x) T^{\rho \sigma}(y)\right]=\rho \delta^{4}(x-y)\left(\eta^{\mu \rho} \eta^{\nu \sigma}+\eta^{\mu \sigma} \eta^{\nu \rho}\right) \mathcal{O}(x)+\ldots
$$

If the theory contains exactly marginal operators, there may be additional contact terms, as well as additional scale anomalies, and the analysis of our sum rule is more complicated. To avoid these complications, we will not consider exactly marginal operators in this work.

\subsection{Polarization tensors}

We now give a precise definition of the polarization tensors appearing in the pseudoamplitudes. From the point of view of graviton scattering amplitudes, a pseudo-amplitude such as eq. (1.3) is not a natural observable. For example, it is not invariant under spacetime diffeormorphisms, the gauge group of gravity. This is closely related to the fact that physical polarization tensors for gravitons are not well-defined Lorentz invariant functions of the graviton momentum [15]. However, we can define Lorentz invariant polarization tensors by allowing them to depend on an additional "reference" momentum. This makes the pseudo-amplitude a well-defined Lorentz invariant observable in the CFT. There is some arbitrariness in the choice of the polarization tensors, but this arbitrariness cancels on both sides of our sum rule.

To define the polarization tensors, we first define spin-1 polarization vectors. We assume that all momenta are massless $\left(p_{i}^{2}=0, i=1,2,3,4\right)$, so these can be written in terms of Weyl spinors as

$$
\epsilon_{+}^{\mu}(p \mid q)=\frac{1}{\sqrt{2}} \frac{\tilde{\lambda} \bar{\sigma}^{\mu} r}{r \lambda}, \quad \epsilon_{-}^{\mu}(p \mid q)=\frac{1}{\sqrt{2}} \frac{\tilde{r} \bar{\sigma}^{\mu} \lambda}{\tilde{\lambda} \tilde{r}},
$$

where $p^{\mu}=\frac{1}{2} \tilde{\lambda} \bar{\sigma}^{\mu} \lambda$ is the 4-momentum of the "massless particle" coupling to the external operator, and $q^{\mu}=\frac{1}{2} \tilde{r} \bar{\sigma}^{\mu} r$ is an arbitrary massless reference momentum. The normalization factors in the denominators ensure that these are properly normalized and scale invariant for any choice of the reference momentum. We then define spin- 2 polarization tensors by

$$
\epsilon_{ \pm}^{\mu \nu}(p \mid q)=\epsilon_{ \pm}^{\mu}(p \mid q) \epsilon_{ \pm}^{\nu}(p \mid q)
$$

\footnotetext{
${ }^{10}$ In the previous subsection, we argued that logarithmic UV divergences in such terms violate conformal invariance.
} 
Practical calculations are simplified by noting that

$$
p_{\mu} \epsilon_{ \pm}^{\mu}(p \mid q)=q_{\mu} \epsilon_{ \pm}^{\mu}(p \mid q)=0,
$$

which uniquely determines the polarization vectors up to a phase, which cancels in our sum rule.

We see that a complete definition of the pseudo-amplitudes appearing in the sum rule requires a choice of reference momentum for the polarization tensors. We define

$$
\begin{aligned}
\mathcal{M}_{++--}\left(p_{1}, p_{2}, p_{3}, p_{4}\right)= & \epsilon_{+}^{\mu_{1} \nu_{1}}\left(p_{1} \mid p_{2}\right) \epsilon_{+}^{\mu_{2} \nu_{2}}\left(p_{2} \mid p_{1}\right) \epsilon_{-}^{\mu_{3} \nu_{3}}\left(p_{3} \mid p_{4}\right) \epsilon_{-}^{\mu_{4} \nu_{4}}\left(p_{4} \mid p_{3}\right) \\
& \times \mathcal{M}_{\mu_{1} \nu_{1} \cdots \mu_{4} \nu_{4}}\left(p_{1}, p_{2}, p_{3}, p_{4}\right),
\end{aligned}
$$

where

$$
\begin{aligned}
& (2 \pi)^{4} \delta^{4}\left(p_{1}+\cdots+p_{4}\right) i \mathcal{M}_{\mu_{1} \nu_{1} \cdots \mu_{4} \nu_{4}}\left(p_{1}, p_{2}, p_{3}, p_{4}\right) \\
& \quad=\int d^{4} x_{1} \cdots d^{4} x_{4} e^{i\left(p_{1} \cdot x_{1}+\cdots+p_{4} \cdot x_{4}\right)}\left\langle 0\left|\mathrm{~T}\left[T_{\mu_{1} \nu_{1}}\left(x_{1}\right) \cdots T_{\mu_{4} \nu_{4}}\left(x_{4}\right)\right]\right| 0\right\rangle .
\end{aligned}
$$

We can define the forward limit in terms of the helicity spinors by

$$
\begin{array}{lll}
\tilde{\lambda}_{1} \rightarrow \lambda_{1}^{*}, & \lambda_{3} \rightarrow \lambda_{1}, & \lambda_{4} \rightarrow \lambda_{2}, \\
\tilde{\lambda}_{2} \rightarrow \lambda_{2}^{*}, & \tilde{\lambda}_{3} \rightarrow-\lambda_{1}^{*}, & \tilde{\lambda}_{4} \rightarrow-\lambda_{2}^{*} .
\end{array}
$$

In this limit, the momenta are real and everything is a function of the two spinors $\lambda_{1,2}$, and we have

$$
\begin{aligned}
\mathcal{M}_{++--}\left(p_{1}, p_{2},-p_{1},-p_{2}\right)= & \epsilon_{+}^{\mu_{1} \nu_{1}}\left(p_{1} \mid p_{2}\right) \epsilon_{+}^{\mu_{2} \nu_{2}}\left(p_{2} \mid p_{1}\right)\left[\epsilon_{+}^{\mu_{3} \nu_{3}}\left(p_{1} \mid p_{2}\right) \epsilon_{+}^{\mu_{4} \nu_{4}}\left(p_{2} \mid p_{1}\right)\right]^{*} \\
& \times \mathcal{M}_{\mu_{1} \nu_{1} \cdots \mu_{4} \nu_{4}}\left(p_{1}, p_{2},-p_{1},-p_{2}\right) .
\end{aligned}
$$

With the definition eq. (2.23), we see that the forward pseudo-amplitude has a positive imaginary part given by the optical theorem, as desired.

We then define the crossed pseudo-amplitude by $2 \leftrightarrow 4$ crossing:

$$
\mathcal{M}_{+--+}\left(p_{1}, p_{2}, p_{3}, p_{4}\right)=\mathcal{M}_{++--}\left(p_{1}, p_{4}, p_{3}, p_{2}\right) .
$$

In the forward limit (2.25), this is given by

$$
\begin{aligned}
\mathcal{M}_{+--+}\left(p_{1}, p_{2},-p_{1},-p_{2}\right)= & \epsilon_{+}^{\mu_{1} \nu_{1}}\left(p_{1} \mid p_{2}\right) \epsilon_{-}^{\mu_{2} \nu_{2}}\left(p_{2} \mid p_{1}\right)\left[\epsilon_{+}^{\mu_{3} \nu_{3}}\left(p_{1} \mid p_{2}\right) \epsilon_{-}^{\mu_{4} \nu_{4}}\left(p_{2} \mid p_{1}\right)\right]^{*} \\
& \times \mathcal{M}_{\mu_{1} \nu_{1} \cdots \mu_{4} \nu_{4}}\left(p_{1}, p_{2},-p_{1},-p_{2}\right),
\end{aligned}
$$

which also has a positive imaginary part.

Changing the reference momentum changes the polarization vectors by a "gauge transformation" $\epsilon^{\mu \nu}(p) \rightarrow \epsilon^{\mu \nu}(p)+p^{(\mu} \xi^{\nu)}$ for some $\xi^{\mu}$ that can depend on $p^{\mu}$ as well as the reference momentum. This gives a contribution to the sum rule proportional to $p_{\mu} \tilde{T}^{\mu \nu}(p)$, the Fourier transform of $\partial_{\mu} T^{\mu \nu}(x)$. This changes the pseudo-amplitude by a contact term, and does not affect the sum rule, as explained in section 2.4 above. In fact, a basis for the transverse traceless polarization tensors is given by

$$
\epsilon_{+}^{\mu}(p) \epsilon_{+}^{\nu}(p), \quad \epsilon_{-}^{\mu}(p) \epsilon_{-}^{\nu}(p), \quad p^{(\mu} \epsilon_{+}^{\nu)}(p), \quad p^{(\mu} \epsilon_{-}^{\nu)}(p), \quad p^{\mu} p^{\nu},
$$


where $\epsilon_{ \pm}^{\mu}(p)$ are the helicity eigenstates used previously. Except for the first two, all of these are "pure gauge," and give pure contact term contributions on the right-hand side of the sum rule. In other words, the sum rule is saturated by contributions from the state $|T\rangle$. We have verified explicitly that these additional "trivial" sum rules are satisfied, providing an additional check on our normalizations and treatment of contact terms.

\section{Evaluation of the sum rule}

In this section, we carry out the evaluation of the sum rule for $c$. We first review the properties of the pseudo-amplitudes that enter into the sum rule. We then outline how conformal Ward identities can be used to determine the contribution of arbitrary operators in terms of their OPE coefficients. The details are relegated to appendix B, including the calculation of the contribution of operators of spin 0 and 2 , and of the energy-momentum tensor. We conclude this section by giving the final form of the sum rule and making some comments about the result.

\subsection{Ingredients for the sum rule}

We first review all the properties of the pseudo-amplitudes $\mathcal{M}_{++--}$and $\mathcal{M}_{+--+}$that are needed to derive the sum rule.

1. The pseudo-amplitudes $\mathcal{M}_{++--}\left(p_{1}, \ldots, p_{4}\right)$ and $\mathcal{M}_{+--+}\left(p_{1}, \ldots, p_{4}\right)$ have a finite real and imaginary part in the massless limit $p_{i}^{2} \rightarrow 0 .{ }^{11}$

2. The resulting massless pseudo-amplitudes are Lorentz-invariant functions of the momenta $p_{1}, \ldots, p_{4} \cdot{ }^{12}$

3. The massless pseudo-amplitudes are real-analytic functions of complex momenta. This follows from the standard analyticity properties of correlation functions in quantum field theory.

4. The pseudo-amplitudes have a finite real and imaginary part in the forward limit $p_{3} \rightarrow-p_{1}, p_{4} \rightarrow-p_{2}$.

5. The crossing symmetry $2 \leftrightarrow 4$ relates the two helicity pseudo-amplitudes. In the forward limit, this implies

$$
\mathcal{M}_{++--}(s)=\mathcal{M}_{+--+}(-s)
$$

6. The imaginary part of the helicity pseudo-amplitudes is a positive sum over CFT states:

$$
\operatorname{Im} \mathcal{M}_{+ \pm-\mp}(s)=\frac{1}{2} \sum_{|\psi\rangle \neq|0\rangle}\left|\left\langle\psi\left|\mathrm{T}\left[\tilde{T}_{+}\left(p_{1}\right) \tilde{T}_{ \pm}\left(p_{2}\right)\right]\right| 0\right\rangle\right|^{2} .
$$

\footnotetext{
${ }^{11}$ Note that these are the only independent helicity amplitudes for our purposes: all others are related to these by complex conjugation or the crossing $1 \leftrightarrow 2$, which act trivially in the forward limit.

${ }^{12}$ Helicity is not Lorentz invariant for massive momenta, so the helicity amplitudes are Lorentz invariant only in the massless limit.
} 
Except for the absence of IR divergences (points 1 and 4), all of these properties were established in the previous section. The absence of IR divergences will be demonstrated for free field theories in section 4 . Together, the above statements imply that

$$
\operatorname{Im} \mathcal{M}_{+ \pm-\mp}(s)=A s^{2}+B s^{2} \ln \frac{\mp s}{\Lambda^{2}}+B^{\prime} s^{2} \ln \frac{ \pm s}{\Lambda^{2}},
$$

where $A, B$ and $B^{\prime}$ are real. The imaginary part of the pseudo-amplitudes is given by the coefficient of $\ln (-s)$, so we can write both $B$ and $B^{\prime}$ as a positive sum over states using the optical theorem. This is our sum rule.

\subsection{Conformal blocks from Ward identities}

We now outline how the Ward identities can be used to compute the contributions of individual operators in the sum over states. (Details are in appendix B.) Because the CFT states are labeled by primary operators, this contribution can be thought of as a conformal block in momentum space. These conformal blocks are essentially the squares of 3-point functions in momentum space, and these are completely fixed up to OPE coefficients by conformal Ward identities.

It is convenient to write the 2- and 3-point functions that we must compute in terms of matrix elements of the states

$$
\left|\mathcal{O}^{\alpha_{1} \cdots \alpha_{\ell}}\right\rangle=\mathcal{O}^{\alpha_{1} \cdots \alpha_{\ell}}(i \epsilon, \overrightarrow{0})|0\rangle
$$

where $\mathcal{O}$ is an arbitrary spin- $\ell$ operator (with $\ell$ even). The operator is inserted at an infinitesimal positive imaginary time $(\epsilon>0)$ to give the proper Wightman ordering of correlation functions. We have

$$
\begin{aligned}
\left\langle 0\left|\tilde{\mathcal{O}}^{\alpha_{1} \cdots \alpha_{\ell}}\left(k^{\prime}\right) \tilde{\mathcal{O}}^{\beta_{1} \cdots \beta_{\ell}}(k)\right| 0\right\rangle= & (2 \pi)^{4} \delta^{4}\left(k^{\prime}+k\right)\left\langle\mathcal{O}^{\alpha_{1} \cdots \alpha_{\ell}}\left|\tilde{\mathcal{O}}^{\beta_{1} \cdots \beta_{\ell}}(k)\right| 0\right\rangle \\
\left\langle 0\left|\tilde{\mathcal{O}}^{\alpha_{1} \cdots \alpha_{\ell}}(k) \mathrm{T}\left[\tilde{T}^{\mu \nu}\left(p_{1}\right) \tilde{T}^{\rho \sigma}\left(p_{2}\right)\right]\right| 0\right\rangle= & (2 \pi)^{4} \delta^{4}\left(k+p_{1}+p_{2}\right) \\
& \times\left\langle\mathcal{O}^{\alpha_{1} \cdots \alpha_{\ell}}\left|\mathrm{T}\left[\tilde{T}^{\mu \nu}\left(p_{1}\right) \tilde{T}^{\rho \sigma}\left(p_{2}\right)\right]\right| 0\right\rangle,
\end{aligned}
$$

and therefore, for physical momenta $k$ we have (see eq. (2.2))

$$
\Pi_{\mathcal{O}}^{\alpha_{1} \cdots \alpha_{\ell}, \beta_{1} \cdots \beta_{\ell}}(k)=\left\langle\mathcal{O}^{\alpha_{1} \cdots \alpha_{\ell}}\left|\mathcal{O}^{\beta_{1} \cdots \beta_{\ell}}(k)\right| 0\right\rangle .
$$

The 2- and 3-point functions appearing in eqs. (3.6) and (3.7) are completely determined by conformal invariance, up to operator normalization and OPE coefficients. We can therefore use Ward identities to completely determine the 3-point functions, again up to OPE coefficients. Specifically, we will need the Ward identities for the conservation and tracelessness of the energy-momentum tensor, as well as the conformal Ward identities. The operator ordering is essential in these matrix elements, and is reflected in the contact term structure, as explained in section 2.4 above. The contact term structure is also reflected in the Ward identities. For example, the conservation Ward identities for the 
3-point functions we need are given in position space by

$$
\begin{aligned}
& \frac{\partial}{\partial x_{1}^{\mu}}\left\langle\mathcal{O}^{\alpha_{1} \cdots \alpha_{\ell}}\left|\mathrm{T}\left[T^{\mu \nu}\left(x_{1}\right) T^{\rho \sigma}\left(x_{2}\right)\right]\right| 0\right\rangle=0, \quad(\mathcal{O} \neq T) \\
& \frac{\partial}{\partial x_{1}^{\mu}}\left\langle T^{\alpha \beta}\left|\mathrm{T}\left[T^{\mu \nu}\left(x_{1}\right) T^{\rho \sigma}\left(x_{2}\right)\right]\right| 0\right\rangle \\
& =\delta^{4}\left(x_{1}-x_{2}\right)\left[\frac{\partial}{\partial x_{2 \nu}}\left\langle T^{\alpha \beta}\left|T^{\rho \sigma}\left(x_{2}\right)\right| 0\right\rangle-\eta^{\nu \rho} \frac{\partial}{\partial x_{2}^{\tau}}\left\langle T^{\alpha \beta}\left|T^{\sigma \tau}\left(x_{2}\right)\right| 0\right\rangle\right. \\
& \left.\quad-\eta^{\nu \sigma} \frac{\partial}{\partial x_{2}^{\tau}}\left\langle T^{\alpha \beta}\left|T^{\rho \tau}\left(x_{2}\right)\right| 0\right\rangle\right],
\end{aligned}
$$

and

$$
\frac{\partial}{\partial z^{\alpha}}\left\langle 0\left|T^{\alpha \beta}(z) \mathrm{T}\left[T^{\mu \nu}\left(x_{1}\right) T^{\rho \sigma}\left(x_{2}\right)\right]\right| 0\right\rangle=0 .
$$

The presence or absence of contact terms on the right-hand sides of these equations can be understood as follows. There are no contact terms between operators with Wightman ordering (see section 2.4 above), so the only possible contact terms in these identities are between the energy-momentum tensors in the time-ordered product. The only operators that can appear in the contact term between two energy-momentum tensors are the identity operator and the energy-momentum tensor itself. ${ }^{13}$ Neither of these contributes a contact term in eqs. (3.8) or (3.10), while the energy-momentum tensor gives rise to the contact terms on the right-hand side of eq. (3.9). When Fourier transformed to momentum space, the contact terms become polynomials in the momenta. This yields the conservation Ward identities given in eqs. (B.42a)-(B.42c) in appendix B. Analogous results hold for the tracelessness and conformal Ward identities.

The procedure to determine the 3-point functions eq. (3.6) is then the following. We write the Ward identities for conservation, tracelessness, and conformal invariance in momentum space, taking care to use the correct contact term structure as explained above. We solve the Ward identities explicitly for the case where $\mathcal{O}$ is a scalar operator or a spin-2 operator (2-index symmetric traceless tensor). The latter case includes the energymomentum tensor, which must be treated with special care.

This in principle gives the result, but we still want to relate the unknown coefficients in momentum space to a conventional definition of the TTO OPE coefficients in position space. For the case where $\mathcal{O}$ is the energy-momentum tensor, the Ward identities in momentum space imply that the contribution of the energy-momentum tensor is proportional to $c$ itself and determining the coefficient. For other operators with generic dimension, there are no contact term ambiguities, and it is straightforward to perform the Fourier transform from position space to momentum space. In practice, since we are only normalizing one or two OPE coefficients, we only need to compute the Fourier transform for several independent scalar contractions of the correlation function, greatly simplifying the algebra. We expect that the results obtained by analytic continuation in the dimensions are correct even for special integer dimensions, with the possible exception of exactly marginal scalar operators, which have additional subtleties.

\footnotetext{
${ }^{13}$ We assume that the theory does not have exactly marginal operators, as discussed in section 2.4.
} 
In this way, we obtain the momentum-space 3-point functions eq. (3.6) in terms of OPE coefficients defined in position space. The calculations are carried out explicitly only for operators with spin 0 or 2 , but the same methods can be used for arbitrary symmetric tensor operators, at the price of additional calculational complexity. The 2-point functions eq. (3.7) are also determined by the momentum space Ward identities in a similar manner.

The final sum rule is obtained by writing sum rules for both $\operatorname{Im} \mathcal{M}_{++--}$and $\operatorname{Im} \mathcal{M}_{+--+}$using the optical theorem eq. (2.6), where the pseudo-amplitudes are defined in terms of the polarization vectors defined in section 2.5. We use the result

$$
\operatorname{Im} \mathcal{M}_{++--}+\operatorname{Im} \mathcal{M}_{+--+}=5 \pi c s^{2},
$$

obtained for example from any of the free field CFTs. We therefore define

$$
\operatorname{Im} \mathcal{M}_{+ \pm-\mp}=5 \pi s^{2} \sum_{\mathcal{O} \neq \mathbb{1}} \sum_{a, b} \lambda_{T T \mathcal{O}}^{(a)} \lambda_{T T \mathcal{O}}^{(b)} f_{a b}^{( \pm)}(\mathcal{O})
$$

so that

$$
c=\sum_{\mathcal{O} \neq \mathbb{1}} \sum_{a, b} \lambda_{T T \mathcal{O}}^{(a)} \lambda_{T T \mathcal{O}}^{(b)}\left[f_{a b}^{(+)}(\mathcal{O})+f_{a b}^{(-)}(\mathcal{O})\right] .
$$

The contribution of the energy-momentum tensor (obtained either from Ward identities, or any of the free-field CFTs) is found to be

$$
\sum_{a, b} \lambda_{T T T}^{(a)} \lambda_{T T T}^{(b)}\left[f_{a b}^{(+)}(T)+f_{a b}^{(-)}(T)\right]=\frac{3}{5} c,
$$

and therefore the final form of our sum rule is

$$
c=\sum_{\mathcal{O} \neq \mathbb{1}, T} \sum_{a, b} \lambda_{T T \mathcal{O}}^{(a)} \lambda_{T T \mathcal{O}}^{(b)} f_{a b}(\mathcal{O}),
$$

where

$$
f_{a b}(\mathcal{O})=\frac{5}{2}\left[f_{a b}^{(+)}(\mathcal{O})+f_{a b}^{(-)}(\mathcal{O})\right] .
$$

For the case where $\mathcal{O}$ is a scalar operator, we have

$$
f(\mathcal{O})=\frac{9 \pi^{3} 2^{2 \Delta+2} \sin ^{2}\left(\frac{\pi}{2} \Delta\right)}{(\Delta-6)^{2}(\Delta-4)^{2} \Delta^{4}(\Delta+2)^{4}} \frac{\Gamma\left(\frac{\Delta-1}{2}\right) \Gamma\left(\frac{\Delta+1}{2}\right)}{\Gamma\left(\frac{\Delta+4}{2}\right)^{2}},
$$

where the normalization of the $\lambda_{T T \mathcal{O}}$ OPE coefficients is defined to be that of ref. [10]. For spin-2 operators there are 2 independent TTO OPE coefficients, and the result is more complicated. It is given in eq. (B.69) in appendix B.

The numerical values of the coefficients are plotted in figure 2 as a function of the dimension $\Delta$ of the operator $\mathcal{O}$, along with asymptotic approximations that are valid for large $\Delta$. 


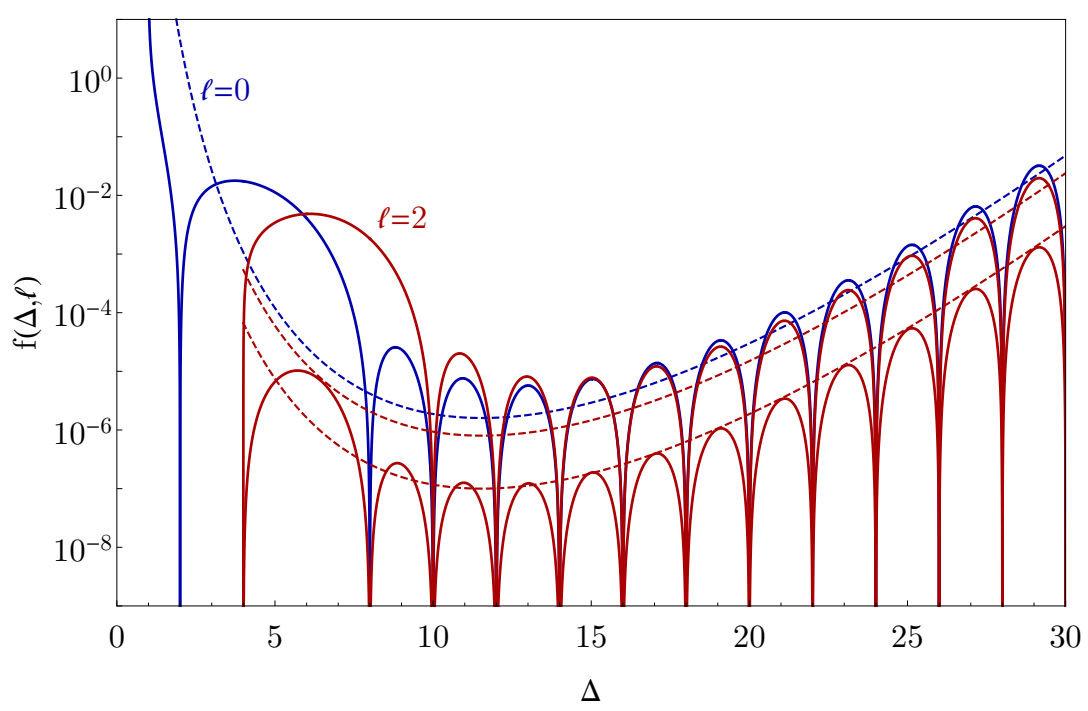

Figure 2. Numerical value of the conformal blocks (3.17) for an operator of spin zero and (B.69) for spin two, showing the eigenvalues of the $2 \times 2$ matrix in the latter case. The dashed lines correspond to the amplitude of the asymptotic expressions (B.67) and (B.70) with the $\sin ^{2}$ factored out.

\subsection{Discussion of results}

We now discuss some features of the functions $f(\mathcal{O})$ appearing in our sum rule eq. (3.15). Some of these features are shared by the sum rule of ref. [3] and the bound of refs. [10, 13].

1. For scalar $\mathcal{O}, f(\mathcal{O})$ diverges as $\Delta \rightarrow 1$ (the unitarity limit):

$$
f(\mathcal{O}) \sim \frac{1}{\Delta-1} .
$$

This means that for any scalar operator with dimension $\Delta=1+\epsilon$, the TTO OPE coefficient must vanish at least as fast as $\sqrt{\epsilon}$ as $\epsilon \rightarrow 0$.

2. For scalar $\mathcal{O}$ we have a double zero as $\Delta \rightarrow 2$ :

$$
f(\mathcal{O}) \sim(\Delta-2)^{2}
$$

This means that operators with dimension 2 do not contribute to the sum rule, and the contribution of operators with dimension near 2 are highly suppressed. This zero ensures that the operator $\phi^{2}$ in free scalar theory does not contribute to the sum rule, despite the fact that the OPE coefficient $T T \phi^{2}$ is nonzero, as explained in section 4 below. In fact, the coefficient of the double zero can be used to check the normalization of $f(\mathcal{O})$, as explained in section C.4.

3. For scalar $\mathcal{O}$, we have double zeros as $\Delta \rightarrow 8,10,12, \ldots$ :

$$
f(\mathcal{O}) \sim(\Delta-(8+2 n))^{2}, \quad n=0,1,2, \ldots
$$


The presence of these zeros can be understood from the fact that the sum rule must be valid in generalized free field theories. A generalized free field theory is obtained from a large- $N$ theory by taking the $N \rightarrow \infty$ limit "from the beginning", as follows. If $c \sim N$, we define $\hat{T}=T / \sqrt{N}$, and our sum rule is

$$
\hat{c}=\text { anomaly coefficient in }\langle\hat{T} \hat{T} \hat{T} \hat{T}\rangle_{\text {con }}=\sum_{\hat{\mathcal{O}}} \lambda_{\hat{T} \hat{T} \hat{\mathcal{O}}}^{2} f(\hat{\mathcal{O}})
$$

where we normalize the operators so that $\langle\hat{\mathcal{O}} \hat{\mathcal{O}}\rangle \sim N^{0}$. The connected correlation function vanishes in the $N \rightarrow \infty$ limit, as does its anomaly, so $\hat{c}=0$. The only operators $\hat{\mathcal{O}}$ appearing in the $\hat{T} \hat{T}$ OPE are double-trace operators $\sim T \partial^{2 n} T$. For such operators the OPE coefficients $\hat{T} \hat{T} \hat{\mathcal{O}}$ are nonzero, so the coefficients $f(\hat{\mathcal{O}})$ must vanish, as we have found from explicit computation. In this sense the sum rule is trivially satisfied for generalized free field theories.

4. It is also interesting to take the large- $N$ limit of our sum rule in a way that keeps the information in the connected correlation function. To do this, we compute our sum rule for large $N$, and take the $N \rightarrow \infty$ limit at the end. At large $N$ a generic single trace operator $\mathcal{O}$ has $\lambda_{T T \mathcal{O}} \sim N$ and $f(\mathcal{O}) \sim 1 /\langle\mathcal{O O}\rangle \sim 1 / N$, so the contribution of every such term in the sum is $\delta c \sim N$, consistent with $c \sim N$. Things are more interesting in a large- $N$ holographic theory. In such a theory all the single trace operators in the TT OPE have dimensions larger than $\Delta_{\text {gap }} \gg 1\left(\Delta_{\text {gap }}\right.$ may grow with $N)$. For a double trace operator $\mathcal{O} \sim T \partial^{2 n} T$, we have $\lambda_{T T \mathcal{O}} \sim N^{2}$ and $\langle\mathcal{O O}\rangle \sim N^{2}$, and $f(\mathcal{O}) \sim 1 / N^{4}$. This gives a contribution $\delta c \sim N^{0}$ to our sum rule, which is negligible in the large- $N$ limit (in agreement with our discussion of the generalized free field limit above). However, our sum rule can still hold in such a large- $N$ theory. For example, it may be saturated at large $N$ by contributions from the single-trace operators with dimensions above $\Delta_{\text {gap }}$. In theories where $\Delta_{\text {gap }}$ grows with $N$, the number of double trace operators below the gap grows with $N$, and it is conceivable that the sum of their contributions is of order $N$. The various scenarios are beyond the scope of our present work, but it is clear that large- $N$ counting alone does not invalidate our sum rule in holographic theories. It would be interesting to see what can be learned from the AdS description of such a theory, and we make some brief comments on this in the conclusions.

5. For $\mathcal{O}$ with spin 2 , both eigenvalues of $f_{a b}(\mathcal{O})$ have double zeros at $\Delta=10,12,14, \ldots$, while at $\Delta=8$ one of the eigenvalue has a double zero. Some or all of these zeros can be understood from the fact that the double trace operators $T \partial^{2+2 n} T$ appear in the spin-2 terms in the OPE of the disconnected part of the $\langle T T T T\rangle$ correlation function. A definite statement would require a classification of the spin- 2 double trace operators appearing in the TT OPE, which we do not attempt here.

6. For $\mathcal{O}$ with spin 2 , both eigenvalues of $f_{a b}(\mathcal{O})$ vanish as $\Delta \rightarrow 4$. One of the eigenvalues is a double zero, the other is a simple zero. These zeros can be understood from free CFTs, as explained in section 4.5 below. 
7. It is interesting to note that even though for a spin-2 operator $f(\mathcal{O})$ has zeros at the unitarity bound, higher-spin conserved currents with $\ell \geq 4$ do contribute to the sum rule. This can be seen from the free field calculations of the next section.

8. In order for the sum over $\mathcal{O}$ to converge, the TTO OPE coefficients must be bounded for large $\Delta$. We have

$$
f(\mathcal{O}) \sim \frac{4^{\Delta}}{\Delta^{16}} \sin ^{2}(\pi \Delta / 2) .
$$

A necessary condition for convergence is therefore

$$
\left|\lambda_{T T \mathcal{O}}\right|^{2} \lesssim \frac{\Delta^{15}}{4^{\Delta}} \frac{1}{\sin ^{2}(\pi \Delta / 2)} .
$$

This is consistent with the implication of the OPE convergence found in refs. [16, 17]. Note that this bound becomes very weak near integer values of $\Delta$.

9. Our function $f(\mathcal{O})$ is similar to a related quantity appearing in the bound derived in ref. [10]:

$$
n_{\mathrm{s}} \geq \sum_{\mathcal{O}=\text { scalar }} \lambda_{\text {TTO }}^{2} f_{\mathrm{CMT}}(\mathcal{O})
$$

while our result gives the bound

$$
n_{\mathrm{s}}+6 n_{\mathrm{f}}+12 n_{\mathrm{v}} \geq \sum_{\mathcal{O}=\text { scalar }} \lambda_{T T \mathcal{O}}^{2} \frac{f(\mathcal{O})}{c_{\text {scalar }}}
$$

The relation between the coefficients in these bounds is

$$
\frac{1}{c_{\text {scalar }}} f(\mathcal{O})=\frac{5}{96}(\Delta-2)^{2} f_{\mathrm{CMT}}(\mathcal{O}) .
$$

The fact that the zeros of the function $f(\mathcal{O})$ are generically double zeros follows from the fact that this function is given by a square of a momentum-space 3-point function, which has zeros at special values of the dimension. The exception is the single zero that occurs for spin- 2 operators at $\Delta=4$. This is due to the fact that the tensor $\Pi_{\mathcal{S}}$ that normalizes the states has a zero eigenvalue at $\Delta=4$.

Because our result gives $c$ as a sum of positive terms, any lower bound on the TTO OPE coefficients immediately gives a lower bound on $c$. This is the kind of result that is usually obtained using the numerical bootstrap, but here it is obtained analytically. To give some idea of the normalization, we present such a "bootstrap plot" in figure 3. The anomaly coefficient $c$ is a natural input parameter for the conformal bootstrap. Given a value for $c$, our sum rule bounds the value of all TTO OPE coefficients, however with "blind spots" at special double-trace values of the scaling dimension as discussed above. It is our hope that this sum rule will be a useful input into the program of bootstrapping the energy-momentum tensor. 


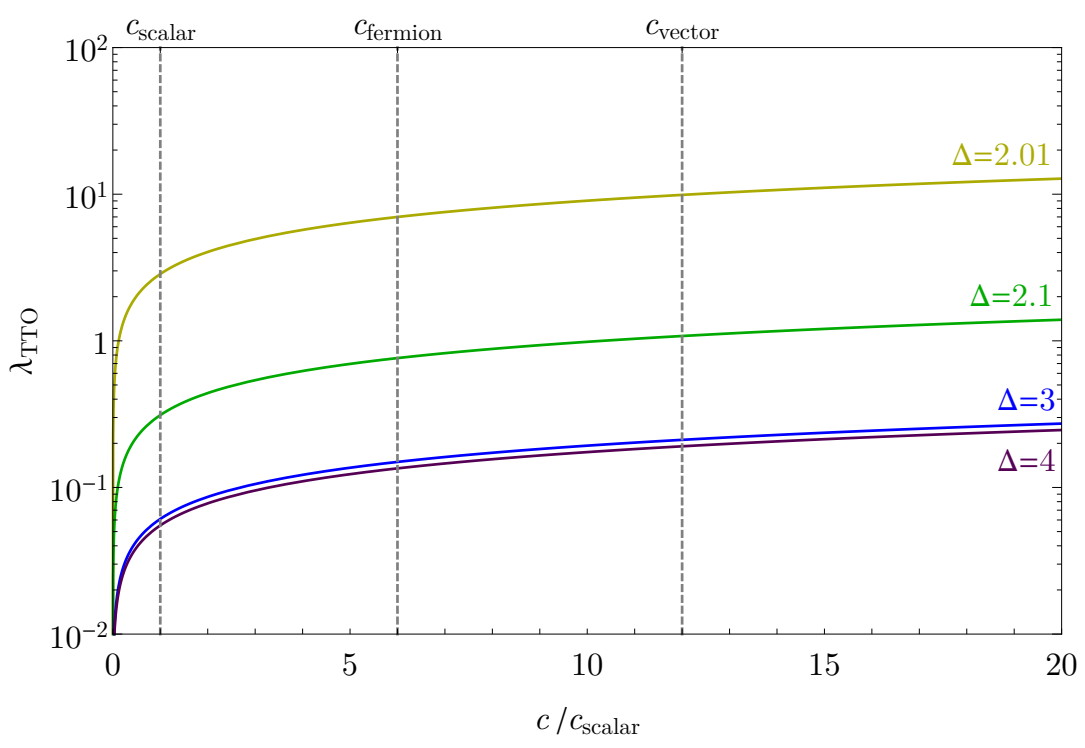

Figure 3. Upper bound on the size of the OPE coefficient for a scalar operator of dimension $\Delta$ entering the $T \times T \mathrm{OPE}$, for a given value of the $c$ anomaly. The bound is weaker for operators of dimension close to 2. This can be alternatively be understood as a lower bound on $c$ for a fixed value of the OPE coefficient.

\section{Free theories}

In this section we investigate our sum rule in the case of free CFTs, namely massless free scalars, fermions, and vectors. In these theories we can check that the pseudo-amplitudes appearing in our sum rule have finite massless and forward limits. We also use free field theories to fix the constant of proportionality between the imaginary part of the pseudoamplitude and $c$, give an independent way of computing the contribution of the energymomentum tensor to the sum rule, and check of the normalization of the contribution of scalar operators.

\subsection{IR finiteness}

As explained in section 3, a crucial assumption in the derivation of our sum rule is that the pseudo-amplitude $\mathcal{M}$ defined in eq. (1.3) is finite under both the massless limits $m^{2} \equiv$ $-p_{i}^{2} \rightarrow 0$ and the forward scattering limit $t \equiv-\left(p_{1}+p_{3}\right)^{2} \rightarrow 0$. It is not sufficient to require that the imaginary part is finite (see section 1 ).

We have verified that this IR finiteness holds for all the three free CFTs. To perform this check, we used the method of expansion by regions [18]. We find that all the regions in all the relevant diagrams are free from IR divergences provided that the polarization tensors are chosen to be transverse and traceless. Details of this computation are given in appendix C. With this check, we know that the sum rule is valid for free theories. 


\subsection{Scattering rates}

We begin by computing the imaginary part of the pseudo-amplitudes $\operatorname{Im} \mathcal{M}_{+ \pm-\mp}(s)$. This is proportional to the total scattering rate $\sigma\left(h_{+} h_{ \pm} \rightarrow \mathrm{CFT}\right)$, where $h$ denotes the graviton field. In free theories, this computation can be carried out using the usual Feynman rules, given in the appendix $\mathrm{C}$. The results for a real scalar are

$$
\begin{aligned}
& 2 \operatorname{Im} \mathcal{M}_{++--}^{\text {scalar }}=\frac{1}{320 \pi} s^{2}, \\
& 2 \operatorname{Im} \mathcal{M}_{+--+}^{\text {scalar }}=\frac{1}{480 \pi} s^{2} .
\end{aligned}
$$

For a free Dirac fermion we have

$$
\begin{aligned}
& 2 \operatorname{Im} \mathcal{M}_{++--}^{\text {fermion }}=\frac{3}{160 \pi} s^{2}, \\
& 2 \operatorname{Im} \mathcal{M}_{+--+}^{\text {fermion }}=\frac{1}{80 \pi} s^{2},
\end{aligned}
$$

and for a free vector theory we have

$$
\begin{aligned}
& 2 \operatorname{Im} \mathcal{M}_{++--}^{\text {vector }}=\frac{3}{80 \pi} s^{2}, \\
& 2 \operatorname{Im} \mathcal{M}_{+--+}^{\text {vector }}=\frac{1}{40 \pi} s^{2} .
\end{aligned}
$$

These result are all consistent with the statement that the sum of the two scattering rates is proportional to the $c$-anomaly, by eq. (A.5). In fact, we can use any of the three free theories described above to fix the constant of proportionality in eq. (3.11).

\subsection{Contributing operators}

We now discuss which operators $\mathcal{O}$ can appear on the right-hand side of the sum rule in the case of free field theories. To get a non-zero contribution, both the OPE coefficient $\lambda_{T T \mathcal{O}}$ and the coefficient $f(\mathcal{O})$ in eq. (1.13) need to be nonzero. In free theories, the energymomentum operator $T$ is parity-even and contains 2 powers of the free fields $\left(\phi, \psi\right.$, or $\left.A_{\mu}\right)$. Therefore, in order to appear in the $T \times T$ OPE, $\mathcal{O}$ must be an $\ell=$ even operator made of either 2 or 4 powers of the free field. If $\mathcal{O}$ has four powers of the field, the 3 -point function $\langle T T \mathcal{O}\rangle$ can be factorized into a product of 2-point functions. In this case, $\lambda_{T T \mathcal{O}}$ can be nonzero only if $\mathcal{O} \sim \partial^{2 n} T T(n \in \mathbb{N})$. Such operators have scaling dimensions $\Delta=8+2 n$. We find in section B that the coefficients $f(\mathcal{O})$ have zeros precisely at these dimensions, which are the "blind spots" of the sum rules. Therefore, no operator with four powers of the field contributes to the sum. We conclude that $\mathcal{O}$ must have 2 powers of the free field.

Another observation is that the scalar operators $\phi^{2}, \bar{\psi} \psi$, and $F_{\mu \nu} F^{\mu \nu}$ do not contribute to the sum rule. This is easy to understand for the fermion and vector theory cases, as chiral symmetry forbids their presence in the $T \times T$ OPE [10]. On the other hand, in the free scalar theory, $\lambda_{T T \phi^{2}}$ is non-zero. However, we find by direct computation in free field theory, or using the results of section $\mathrm{B}$, that $f\left(\phi^{2}\right)$ is zero in this case.

To summarize, the contributing operators $\mathcal{O}$ are built from two powers of the field and spin-even, but not $\phi^{2}, \bar{\psi} \psi$, or $F_{\mu \nu} F^{\mu \nu}$. 


\subsection{A complete example: the free scalar}

We now verify the sum rule explicitly in the free scalar theory. We showed above that the primary operators contributing to the sum rule have the form $\mathcal{O}^{\alpha_{1} \cdots \alpha_{\ell}} \sim \phi \partial^{\alpha_{1}} \cdots \partial^{\alpha_{\ell}} \phi$ with $\ell$ even. The primary operators are easily seen to be symmetric traceless spin- $\ell$ tensors. Because they have dimension $\Delta=\ell+2$, they saturate the unitarity bounds, and are therefore conserved currents.

Partial wave expansion. A consequence of $\mathcal{O}^{\alpha_{1} \cdots \alpha_{\ell}}$ being conserved currents is that the sum rule expansion coincides with the usual partial wave expansion. This is because the three-point functions must satisfy the Ward identity

$$
\left(p_{1}+p_{2}\right)_{\alpha_{i}}\left\langle\mathcal{O}^{\alpha_{1} \cdots \alpha_{\ell}}\left|\mathrm{T}\left[\tilde{T}_{1}\left(p_{1}\right) \tilde{T}_{2}\left(p_{2}\right)\right]\right| 0\right\rangle=0 .
$$

In the center-of-mass frame where $\left(p_{1}+p_{2}\right)_{\alpha}=\sqrt{s}(1, \overrightarrow{0})$, this Ward identity implies that only the spatial components of the three-point function are non-zero, i.e. those with tensor indices $\alpha_{i}=1,2,3$. Therefore, the three-point function forms a spin- $\ell$ representation of the spatial rotation group $\mathrm{SO}(3)$. There is no $s$-wave contribution, corresponding to the fact that the operator $\phi^{2}$ does not contribute to the pseudo-amplitude.

The correspondence between the OPE and the partial wave expansion can be used to compute all of the contributions to the sum rule in the free scalar case. The imaginary part of the pseudo-amplitude is computed as the scattering rate of the process $h h \rightarrow \phi \phi$, whose amplitude $\mathcal{M}_{h h \rightarrow \phi \phi}(\theta, \varphi)$ can be expanded in terms of the spherical harmonics $Y_{\ell m}(\theta, \varphi)$, where $\theta$ and $\varphi$ are the scattering angles in the center-of-mass frame. Then each spherical harmonic corresponds exactly to one operator of the free scalar theory. Concretely, we consider the cases where the initial gravitons have helicities ++ and +- . We obtain (see section C.3 for details):

$$
\begin{aligned}
\mathcal{M}_{h_{+} h_{+} \rightarrow \phi \phi}(\theta, \varphi) & =\frac{s}{16}\left(3 \cos ^{2} \theta-1\right) \\
& =\frac{s}{4} \sqrt{\frac{\pi}{5}} Y_{20}(\theta, \varphi) \\
\mathcal{M}_{h_{+} h_{-} \rightarrow \phi \phi}(\theta, \varphi) & =\frac{s}{16} e^{4 i \varphi}\left(1-\cos ^{2} \theta\right) \\
& =6 \sqrt{\pi} s \sum_{\text {even } \ell=4}^{\infty} \sqrt{(2 \ell+1) \frac{(\ell-4) !}{(\ell+4) !}} Y_{\ell 4}(\theta, \varphi) .
\end{aligned}
$$

From eq. (4.5a), we see that for initial helicity ++ , only spin- 2 operators contribute to the scattering rate. In the free scalar theory, this corresponds to the contribution of energymomentum tensor, so $T$ saturates this scattering rate. This result can also be obtained by direct computation of the $T$ contribution, as we show below. Using eq. (4.1a), this shows that $T$ contributes as $\frac{3}{5} c$ in our sum rule. As we will explain in section 4.5 , this result can be extended to general CFTs.

For initial helicity +- eq. (4.5b) implies that an infinite number of operators with even spin $\ell \geq 4$ contribute to the rate. In fact, we can identify the contribution of each operator 
to be

$$
\begin{aligned}
\left|\mathcal{M}_{T_{+} T_{-} \rightarrow \phi^{2} \partial^{\ell}}\right|^{2} & =\frac{1}{\pi} \int \frac{d \Omega}{4 \pi}\left|\mathcal{M}_{h_{+} h_{-} \rightarrow \phi \phi}(\theta, \varphi)\right|^{2}=\frac{9}{\pi} s^{2}(2 \ell+1) \frac{(\ell-4) !}{(\ell+4) !} \\
& =6(2 \ell+1) \frac{6 !(\ell-4) !}{(\ell+4) !} \times\left(2 \operatorname{Im} \mathcal{M}_{+-}^{\text {scalar }}\right),
\end{aligned}
$$

for $\ell=4,6,8, \ldots$

OPE. Now we use the OPE eq. (1.11) and compare it with the results of the partial wave expansion. The lowest-dimensional operator that appears in the sum is the energymomentum tensor. According to eq. (2.1), its contribution is

$$
\begin{aligned}
\left|\mathcal{M}_{T_{1} T_{2} \rightarrow T}\left(p_{1}, p_{2}\right)\right|^{2}= & {\left[\Pi_{T}^{-1}\left(p_{1}+p_{2}\right)\right]_{\alpha_{1} \alpha_{2}, \beta_{1} \beta_{2}} } \\
& \times\left\langle 0\left|\overline{\mathrm{T}}\left[\tilde{T}_{1}\left(-p_{1}\right) \tilde{T}_{2}\left(-p_{2}\right)\right]\right| T^{\alpha_{1} \alpha_{2}}\right\rangle\left\langle T^{\beta_{1} \beta_{2}}\left|\mathrm{~T}\left[\tilde{T}_{1}\left(p_{1}\right) \tilde{T}_{2}\left(p_{2}\right)\right]\right| 0\right\rangle .
\end{aligned}
$$

We compute the three-point function $\left\langle T^{\beta_{1} \beta_{2}}\left|\mathrm{~T}\left[\tilde{T}_{1}\left(p_{1}\right) \tilde{T}_{2}\left(p_{2}\right)\right]\right| 0\right\rangle$ and the two-point function $\left[\Pi_{T}\left(p_{1}+p_{2}\right)\right]^{\alpha_{1} \alpha_{2}, \beta_{1} \beta_{2}} \sim\left\langle 0\left|\tilde{T}^{\alpha_{1} \alpha_{2}}\left(-p_{1}-p_{2}\right) \tilde{T}^{\beta_{1} \beta_{2}}\left(p_{1}+p_{2}\right)\right| 0\right\rangle$ by evaluating the contributing Feynman diagrams, which are shown respectively in figures 4 and 5. Note that these loop diagrams are all UV finite due to the presence of Wightman propagators, marked in red in the figures. A straightforward (but tedious) calculation yields

$$
\begin{aligned}
\left|\mathcal{M}_{T_{1} T_{2} \rightarrow T}\right|^{2}= & \frac{s^{2}}{135 \times 64 \pi} \epsilon_{1}^{\mu_{1} \nu_{1}} \epsilon_{2}^{\mu_{2} \nu_{2}}\left(\epsilon_{1}^{\mu_{3} \nu_{3}}\right)^{*}\left(\epsilon_{2}^{\mu_{4} \nu_{4}}\right)^{*} \eta_{\mu_{1} \mu_{2}} \eta_{\mu_{3} \mu_{4}} \\
& \times\left[64\left(\eta_{\nu_{1} \nu_{3}} \eta_{\nu_{2} \nu_{4}}+\eta_{\nu_{1} \nu_{4}} \eta_{\nu_{2} \nu_{3}}\right)-37 \eta_{\nu_{1} \nu_{2}} \eta_{\nu_{3} \nu_{4}}\right],
\end{aligned}
$$

which separates into the two helicity structures as

$$
\begin{aligned}
\left|\mathcal{M}_{T_{+} T_{+} \rightarrow T}\right|^{2} & =\frac{s^{2}}{320 \pi}, \\
\left|\mathcal{M}_{T_{+} T_{-} \rightarrow T}\right|^{2} & =0
\end{aligned}
$$

As anticipated from the partial wave expansion, we see indeed that the $T$ contribution saturates the scattering rate for initial helicities ++ , and gives no contribution in the case +- .

Contributions from the other operators can be evaluated in the same way. For example, it can be verified that the scalar operator $\phi^{2}$ does not contribute to either helicity structure, and that the spin- 4 operator $\partial^{4} \phi^{2}$ gives

$$
\begin{aligned}
& \left|\mathcal{M}_{T_{+} T_{+} \rightarrow \partial^{4} \phi^{2}}\right|^{2}=0 \\
& \left|\mathcal{M}_{T_{+} T_{-} \rightarrow \partial^{4} \phi^{2}}\right|^{2}=\frac{9}{4480 \pi} s^{2}=\frac{27}{28} \times\left(2 \operatorname{Im} \mathcal{M}_{+-}^{\text {scalar }}\right) .
\end{aligned}
$$

Note that the fractional contribution of the spin-four operator precisely matches eq. (4.6) with $\ell=4$. If we use the partial expansion further, we can obtain the contribution of higher-spin operators:

$$
\frac{\left|\mathcal{M}_{T_{+} T_{-} \rightarrow \phi^{2} \partial^{\ell}}\right|^{2}}{2 \operatorname{Im} \mathcal{M}_{+-}^{\text {scalar }}}=\frac{27}{28}, \frac{13}{420}, \frac{17}{4620}, \cdots \quad \text { for } \ell=4,6,8, \ldots
$$




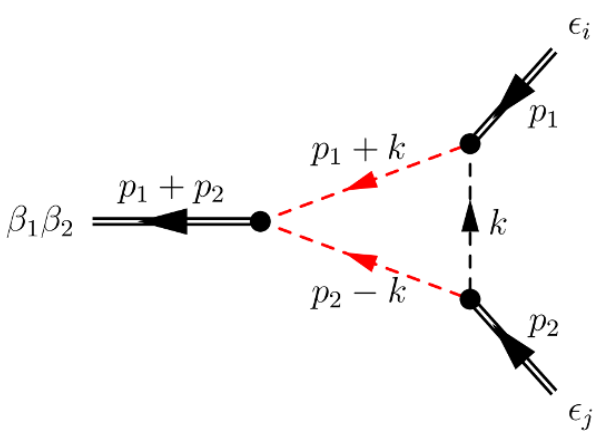

(a)

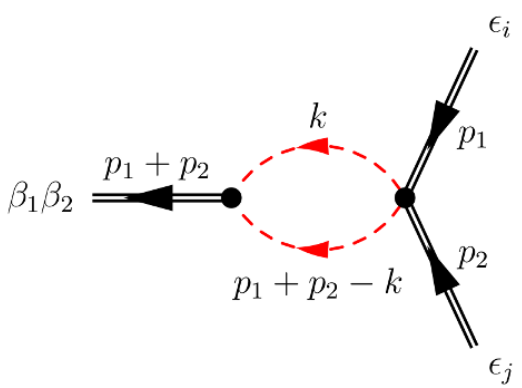

(b)

Figure 4. Feynman diagrams for the three-point function $\left\langle T^{\beta_{1} \beta_{2}}\left|\mathrm{~T}\left[\tilde{T}_{i}\left(p_{1}\right) \tilde{T}_{j}\left(p_{2}\right)\right]\right| 0\right\rangle$ in the free scalar theory. Here we use straight double lines for $T$ operators and dashed singled lines for scalars. Red lines indicate Wightman propagators, black lines ordinary Feynman propagators.

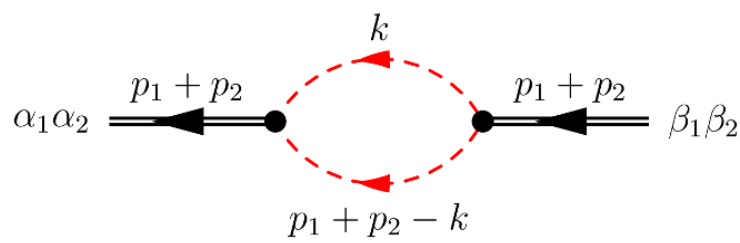

Figure 5. Feynman diagram for two-point function $\left\langle 0\left|\tilde{T}^{\alpha_{1} \alpha_{2}}\left(-p_{1}-p_{2}\right) \tilde{T}^{\beta_{1} \beta_{2}}\left(p_{1}+p_{2}\right)\right| 0\right\rangle$ in the free scalar theory. Straight double lines are for the $T$ operator and dashed singled lines for scalars. Red lines indicate Wightman propagators.

We can check that this converges to the expected value. We can also check the rate of convergence:

$$
\sum_{\text {even } \ell=4}^{\ell_{\max }} \frac{\left|\mathcal{M}_{T_{+} T_{-} \rightarrow \phi^{2} \partial^{\ell}}\right|^{2}}{2 \operatorname{Im} \mathcal{M}_{+-}^{\text {scalar }}}-1=-\frac{6 !\left(\ell_{\max }-2\right) !}{\left(\ell_{\max }+4\right) !} \sim \ell_{\max }^{-6} .
$$

The convergence of this sum is very fast, but not exponentially fast, unlike the positionspace OPE at separated points $[16,17]$.

\section{5 $T$ contribution in general CFTs}

The contribution of the energy-momentum tensor to the scattering rates can be computed similarly in the theories of free fermions and free vectors, and we also find in both cases that it saturates the ++ scattering rate, and therefore gives a contribution of $3 c / 5$ to the sum rule (3.12).

In fact, we can argue that this result extends to arbitrary CFTs, as follows. The tensor structures of the 3 free CFTs form a basis for the 3 independent tensor structures in the TTT 3-point function. That is, we can write the 3-point function in a general CFT as

$$
\langle T T T\rangle=n_{\mathrm{s}}\langle T T T\rangle_{\mathrm{s}}+n_{\mathrm{f}}\langle T T T\rangle_{\mathrm{f}}+n_{\mathrm{v}}\langle T T T\rangle_{\mathrm{v}}
$$

where the subscripts s, f, v refer to real scalars, Dirac fermions, and free vectors. In this way $n_{\mathrm{s}}, n_{\mathrm{f}}$, and $n_{\mathrm{v}}$ become the OPE coefficients in general CFTs. 
In a free theory of real scalars, Dirac fermions, and vectors, these OPE coefficients simply count the number of each type of free field. ${ }^{14}$ In such theories, there is a separate conserved 2-index symmetric tensor for each field, while the energy-momentum tensor is given by the sum of these:

$$
T=T_{\mathrm{s}}+T_{\mathrm{f}}+T_{\mathrm{v}}
$$

where the sum over the different free fields is implicit. The states created by these conserved tensors are mutually orthogonal, so we have (see eq. (3.13))

$$
\begin{aligned}
& \sum_{\mathcal{O}}=T_{\mathrm{s}}, T_{\mathrm{f}}, T_{\mathrm{v}} \\
& =\sum_{a, b} \lambda_{T T \mathcal{O}}^{(a)} \lambda_{T T \mathcal{O}}^{(b)}\left[f_{a b}^{(+)}(\mathcal{O})+f_{\text {TT }}^{(-)}(\mathcal{O})\right] \\
& =\frac{3}{5} c_{\text {free }}^{(b)}\left[f_{a b}^{(+)}(T)+f_{a b}^{(-)}(T)\right]
\end{aligned}
$$

where the function $c_{\text {free }}$ is given in eq. (A.5). On the other hand, for general CFT, we have

$$
\sum_{a, b} \lambda_{T T T}^{(a)} \lambda_{T T T}^{(b)}\left[f_{a b}^{(+)}(T)+f_{a b}^{(-)}(T)\right]=\frac{\text { quadratic function of } n_{\mathrm{s}}, n_{\mathrm{f}}, n_{\mathrm{v}}}{C_{T}}
$$

This function of the three OPE coefficients $n_{\mathrm{s}}, n_{\mathrm{f}}, n_{\mathrm{v}}$ must reduce to the right-hand side of eq. (4.15) at non-negative integer values. The only possibility is for the numerator on the right-hand side of eq. (4.16) to be proportional to $c^{2}$, so that eq. (4.15) to holds for general CFTs. Furthermore, spin-2 operators with $\Delta=d=4$ that are not the energy-momentum tensor must give a vanishing contribution to the sum rule. Both of these features are also found in the computation of the previous section, which was based on the Ward identities in a general CFT.

\section{Conclusions and outlook}

We have presented a sum rule for $c$ in 4 D CFTs based on inserting a complete set of states in a 4-point function of energy-momentum tensors in Minkowski momentum space. This 4point function can be viewed as a contribution to a graviton-graviton scattering amplitude, and the completeness relation is a version of the optical theorem. The sum rule can also be thought of as an OPE, since the intermediate states are labeled by primary operators. This work realizes the idea of ref. [3] relating scale (dilatation) anomaly coefficients to a positive sum over states. We have given explicit expressions for the coefficients for operators of spin 0 and 2, and showed that our results satisfy a number of consistency checks.

Our derivation of the sum rule depends on the unproven assumption that the pseudoamplitude we study is free of IR divergences in the massless and forward limits. This has been checked only in the case of free field CFTs, and a better understanding of this question would be very helpful. Conceptually, the scale anomaly can be thought of as the

\footnotetext{
${ }^{14} \mathrm{~A}$ free Weyl fermion corresponds to $n_{\mathrm{f}}=\frac{1}{2}$ in our conventions.
} 
dependence on a UV regulator, which should have nothing to do with IR divergences. It may be worthwhile to look for a different derivation of the sum rule in which IR divergences are not an issue.

The sum rule is a relationship among CFT data of the same form as the bootstrap equations, specifically the crossing relations for the $\langle T T T T\rangle$ correlation function. The strategy of the numerical bootstrap is to numerically identify linear combinations of bootstrap constraints such that the remainder from omitted operators has a definite sign, so that neglecting the remainder gives rigorous inequalities. Our sum rule is a relation of this kind where all terms are positive as a direct consequence of unitarity. Although the methods used here to derive the sum rule are very special to the $4 \mathrm{D}$ scale anomaly we have studied, it suggests that other positive sum rules may be obtained analytically. More prosaically, we hope that our sum rule will prove to be a useful constraint in the program of bootstrapping the energy-momentum tensor. It remains to be seen whether this constraint is redundant with the constraints that are accessible via the numerical bootstrap.

There are a number of directions for future work. As already mentioned above, we would like to have a better understanding of the possible IR divergences in the 4-point functions of energy-momentum tensors. It would be very interesting to test our sum rule in some specific CFTs, but this requires a 4-dimensional CFT where the TTO OPE coefficients are all known, as well as extending our calculation of the coefficients $f(\mathcal{O})$ to operators of arbitrary spin. Checking the IR convergence in some case requires knowing the 4-point function of energy-momentum tensors in momentum space. We are not aware of any theories in the literature where these results are available, but perhaps this work can serve as a motivation to compute these quantities, for example in $\mathcal{N}=4$ super Yang-Mills theory at large $N$. It may also be interesting to consider our sum rule in holographic theories. As discussed in the body of the paper, in such theories the contribution of individual single double-trace operators (corresponding to supergravity states in $\mathrm{AdS}$ ) is negligible in the large- $N$ limit, but it is possible that these states add up to give a sizeable contribution. If not, it would imply that our sum rule requires contributions from the single trace operators above the dimension gap (corresponding to string states in AdS), even for a large gap. In theories with maximal gap ("pure gravity" in AdS) this would imply that black hole states make important contributions to $c$. The ideas in this paper can also be applied to other kinds of scale anomalies in CFTs, for example in theories with global symmetries, supersymmetry, or exactly marginal operators in various spacetime dimensions. We can also try to extend this work to find a sum rule for the $a$-anomaly in $4 \mathrm{D}$ CFT. More generally, our work illustrates the usefulness of momentum-space techniques in the study of conformal field theory, and we hope that these will find further applications in the study of quantum field theory.

\section{Acknowledgments}

We have benefited from discussions and encouragement from many people, including L. Dixon, A. Dymarsky, J. Kaplan, M. Mojaza, J. Penedones, R. Rattazzi, A. Vichi, and M. Walters. M.G. is supported by the Swiss National Science Foundation through the NCCR SwissMAP and formerly under grant number P300P2154559. X.L. and M.A.L. are supported by the Department of Energy under grant DE-FG02-91ER406746. 


\section{A Conventions}

We use mostly plus spacetime metric and the GR conventions of Wald [19]. The energymomentum tensor is defined by differentiation with respect to the metric

$$
\frac{\delta W\left[g_{\mu \nu}\right]}{\delta g_{\mu \nu}(x)}=\frac{1}{2} \sqrt{-g}\left\langle T^{\mu \nu}(x)\right\rangle_{g}
$$

where $W\left[g_{\mu \nu}\right]$ is the quantum effective action. The anomaly under a Weyl transformation $\delta g_{\mu \nu}=2 \sigma g_{\mu \nu}$ is given by

$$
\delta_{\sigma} W\left[g_{\mu \nu}\right]=\int d^{4} x \sigma\left[c W^{\mu \nu \rho \sigma} W_{\mu \nu \rho \sigma}+a E_{4}\right],
$$

where the square of the Weyl tensor is given by

$$
W^{\mu \nu \rho \sigma} W_{\mu \nu \rho \sigma}=R^{\mu \nu \rho \sigma} R_{\mu \nu \rho \sigma}-2 R^{\mu \nu} R_{\mu \nu}+\frac{1}{3} R^{2},
$$

and the Euler density by

$$
E_{4}=R^{\mu \nu \rho \sigma} R_{\mu \nu \rho \sigma}-4 R^{\mu \nu} R_{\mu \nu}+R^{2} .
$$

With this normalization, in free field theories we have [20]

$$
\begin{aligned}
& c_{\text {free }}=\frac{1}{(4 \pi)^{2}} \frac{n_{\mathrm{s}}+6 n_{\mathrm{f}}+12 n_{\mathrm{v}}}{120}, \\
& a_{\text {free }}=\frac{1}{(4 \pi)^{2}} \frac{n_{\mathrm{s}}+11 n_{\mathrm{f}}+62 n_{\mathrm{v}}}{360},
\end{aligned}
$$

where $n_{\mathrm{s}}$ is the number of real scalars, $n_{\mathrm{f}}$ is the number of Dirac fermions, and $n_{\mathrm{v}}$ is the number of vectors. The normalization of the 2-point function of energy-momentum tensors $C_{T}$ is defined in eq. (B.8), and is related to $c$ by

$$
C_{T}=\frac{640}{\pi^{2}} c
$$

\section{B Computation of the conformal blocks in momentum space}

In this appendix we compute the "conformal blocks" $f(\mathcal{O})$ that appear as coefficients in our sum rule eq. (1.13). We will obtain explicit results for scalar operators $(\ell=0)$ and for traceless symmetric spin- 2 tensors $(\ell=2)$, including the energy-momentum tensor itself. Specifically, we compute the Wightman 2- and 3-point functions (see eqs. (3.4), (3.5), and (3.6))

$$
\left\langle\mathcal{O}^{\alpha_{1} \ldots \alpha_{\ell}}\left|\tilde{\mathcal{O}}^{\beta_{1} \ldots \beta_{\ell}}(k)\right| 0\right\rangle, \quad\left\langle\mathcal{O}^{\alpha_{1} \ldots \alpha_{\ell}}\left|\mathrm{T}\left[\tilde{T}^{\mu \nu}\left(p_{1}\right) \tilde{T}^{\rho \sigma}\left(p_{2}\right)\right]\right| 0\right\rangle .
$$

The method used here is general and can in principle be used to compute the functions $f(\mathcal{O})$ for other types of operators, but the complexity of the calculation increases significantly for higher representations.

This appendix is organized as follows. We first state our conventions for the normalization of operators and OPE coefficients. We then compute the 2- and 3-point functions where the energy-momentum tensor is replaced by scalar operators; this amounts to reviewing the results of ref. [3]. We then use Ward identities to fix completely the correlators of operators with spin, up to a few coefficients. Finally we compute these coefficients in terms of OPE coefficients defined in position space using the results for scalar correlators. 


\section{B.1 Position space 2-point functions and normalization of operators}

For scalar operators, we use the standard normalization of the Wightman two-point function

$$
\langle\mathcal{O}|\mathcal{O}(x)| 0\rangle=\frac{1}{\left(x_{\mathrm{W}}^{2}\right)^{\Delta}},
$$

where the Wightman ordering is imposed by an $i \epsilon$ prescription (see eq. (3.4))

$$
|\mathcal{O}\rangle=\mathcal{O}(i \epsilon, \overrightarrow{0})|0\rangle
$$

which implies the standard Wightman $i \epsilon$ prescription

$$
x_{\mathrm{W}}^{2} \equiv-\left(x^{0}+i \epsilon\right)^{2}+\vec{x}^{2} .
$$

Note that $x_{\mathrm{W}}^{2} \neq(-x)_{\mathrm{W}}^{2}$ due to the $i \epsilon$ prescription. We will drop the subscript $\mathrm{W}$ hereafter, unless it is not clear from the context.

We also consider traceless symmetric spin-two operators, which we denote by $\mathcal{S}^{\mu \nu}$. The conventional normalization for these is

$$
\left\langle\mathcal{S}^{\mu \nu}\left|\mathcal{S}^{\rho \sigma}(x)\right| 0\right\rangle=\frac{\mathcal{I}^{\mu \nu \rho \sigma}(x)}{\left(x^{2}\right)^{\Delta}}
$$

where

$$
\mathcal{I}^{\mu \nu \rho \sigma}(x)=\frac{1}{2}\left[\mathcal{I}^{\mu \rho}(x) \mathcal{I}^{\nu \sigma}(x)+\mathcal{I}^{\mu \sigma}(x) \mathcal{I}^{\nu \rho}(x)\right]-\frac{1}{d} \eta^{\mu \nu} \eta^{\rho \sigma}
$$

and

$$
\mathcal{I}^{\mu \nu}(x)=\eta^{\mu \nu}-\frac{2 x^{\mu} x^{\nu}}{x^{2}}
$$

Note that we have kept the space-time dimension $d$ general. We will continue this as far as possible in this appendix, although we are interested in $d=4$ at the end.

The normalization of the energy-momentum tensor is special, since it is fixed by the fact that it defines the conserved energy and momentum. We therefore have

$$
\left\langle T^{\mu \nu}\left|T^{\rho \sigma}(x)\right| 0\right\rangle=C_{T} \frac{\mathcal{I}^{\mu \nu \rho \sigma}(x)}{\left(x^{2}\right)^{d}} .
$$

The constant $C_{T}$ is related to $c$ by conformal Ward identities, see eq. (A.7).

\section{B.2 Position space 3-point functions and OPE coefficients}

Now that we have fixed the normalization of operators, we can define the OPE coefficients in terms of the tensor structures that enter the 3-point function eq. (3.6). We mostly follow the conventions of ref. [10], which builds on the seminal works of refs. [21, 22] (see also ref. [23]). The 3-point functions can be written in terms of the quantities

$$
\begin{aligned}
V_{1}^{\mu} & =\frac{x_{1}^{2} x_{12}^{\mu}-x_{21}^{2} x_{1}^{\mu}}{x_{2}^{2}}, \\
V_{2}^{\mu} & =\frac{x_{2}^{2} x_{12}^{\mu}+x_{21}^{2} x_{2}^{\mu}}{x_{1}^{2}}, \\
V_{3}^{\mu} & =\frac{x_{2}^{2} x_{1}^{\mu}-x_{1}^{2} x_{2}^{\mu}}{x_{21}^{2}},
\end{aligned}
$$


and the 2-index tensors

$$
\begin{aligned}
& H_{12}^{\mu \nu}=x_{21}^{2} \eta^{\mu \nu}-2 x_{12}^{\mu} x_{12}^{\nu}, \\
& H_{13}^{\mu \nu}=x_{1}^{2} \eta^{\mu \nu}-2 x_{1}^{\mu} x_{1}^{\nu}, \\
& H_{23}^{\mu \nu}=x_{2}^{2} \eta^{\mu \nu}-2 x_{2}^{\mu} x_{2}^{\nu},
\end{aligned}
$$

where $x_{i j} \equiv x_{i}-x_{j}$. For a traceless symmetric tensor operator $\mathcal{O}^{\alpha_{1} \ldots \alpha_{\ell}}$ with scaling dimension $\Delta$ and spin $\ell$, conformal invariance requires the position-space correlation function (compare eq. (B.1)) to be of the form

$$
\left\langle\mathcal{O}^{\alpha_{1} \ldots \alpha_{\ell}}\left|T^{\mu \nu}\left(x_{1}\right) T^{\rho \sigma}\left(x_{2}\right)\right| 0\right\rangle=\frac{\text { (tensors built out of the } \left.V_{i} \text { and } H_{i j}\right)}{\left(x_{1}^{2}\right)^{(\Delta+\ell) / 2}\left(x_{2}^{2}\right)^{(\Delta+\ell) / 2}\left(x_{21}^{2}\right)^{d+2-(\Delta+\ell) / 2}},
$$

where the numerator is such that the $V_{i}$, and $H_{i j}$ have indices corresponding respectively to the operators inserted at the points $x_{i}$ and $x_{j}$ (identifying $x_{3}=0$ ). The examples $\ell=0,2$ will now be discussed in detail.

For a scalar operator $\mathcal{O}$, the most general form of the three-point function consistent with conformal symmetry is

$$
\left\langle\mathcal{O}\left|T^{\mu \nu}\left(x_{1}\right) T^{\rho \sigma}\left(x_{2}\right)\right| 0\right\rangle=\frac{t^{\mu \nu \rho \sigma}}{\left(x_{1}^{2}\right)^{\Delta / 2}\left(x_{2}^{2}\right)^{\Delta / 2}\left(x_{21}^{2}\right)^{d+2-\Delta / 2}}
$$

where the tensor $t^{\mu \nu \rho \sigma}$ is a linear combination of three terms,

$$
\begin{aligned}
t^{\mu \nu \rho \sigma}= & \frac{1}{4}\left[\alpha_{1} V_{1}^{\mu} V_{1}^{\nu} V_{2}^{\rho} V_{2}^{\sigma}+\alpha_{2} H_{12}^{\mu \rho} V_{1}^{\nu} V_{2}^{\sigma}\right. \\
& \left.+\alpha_{3} H_{12}^{\mu \rho} H_{12}^{\nu \sigma}+\text { permutations - traces }\right] .
\end{aligned}
$$

Permutations and traces are understood to be among indices of the same operators, e.g. $(\mu \leftrightarrow \nu)$ and $(\rho \leftrightarrow \sigma)$. The three coefficients $\alpha_{1}, \alpha_{2}$ and $\alpha_{3}$ are not independent: requiring conservation of the energy-momentum tensor adds 2 constraints, so that the correlator eventually depends on a single OPE coefficient. A solution to these constraints is for instance the choice of ref. [10],

$$
\begin{aligned}
& \alpha_{1}=\lambda_{T T \mathcal{O}}, \\
& \alpha_{2}=\frac{2(d-1)(\Delta-d)-4}{(d-2)(\Delta+2)} \lambda_{T T \mathcal{O}}, \\
& \alpha_{3}=\frac{(d-1)(\Delta-d)^{2}-2 d}{(d-2) \Delta(\Delta+2)} \lambda_{T T \mathcal{O}} .
\end{aligned}
$$

Note that all three coefficients remain of order unity even when $\Delta$ is large.

In the case of a traceless symmetric spin- 2 operator, the most general conformal invariant correlator has the form

$$
\left\langle\mathcal{S}^{\alpha \beta}\left|T^{\mu \nu}\left(x_{1}\right) T^{\rho \sigma}\left(x_{2}\right)\right| 0\right\rangle=\frac{t^{\mu \nu \rho \sigma \alpha \beta}}{\left(x_{1}^{2}\right)^{\Delta / 2+1}\left(x_{2}^{2}\right)^{\Delta / 2+1}\left(x_{21}^{2}\right)^{d+1-\Delta / 2}},
$$


where

$$
\begin{aligned}
t^{\mu \nu \rho \sigma \alpha \beta}= & \frac{1}{8}\left[\alpha_{1} V_{1}^{\mu} V_{1}^{\nu} V_{2}^{\rho} V_{2}^{\sigma} V_{3}^{\alpha} V_{3}^{\beta}+\alpha_{2}\left(H_{13}^{\mu \alpha} V_{2}^{\rho}+H_{23}^{\rho \alpha} V_{1}^{\mu}\right) V_{1}^{\nu} V_{2}^{\sigma} V_{3}^{\beta}\right. \\
& +\alpha_{3} H_{12}^{\mu \rho} V_{1}^{\nu} V_{2}^{\sigma} V_{3}^{\alpha} V_{3}^{\beta}+\alpha_{4}\left(H_{13}^{\mu \alpha} V_{2}^{\rho}+H_{23}^{\rho \alpha} V_{1}^{\mu}\right) H_{12}^{\nu \sigma} V_{3}^{\beta} \\
& +\alpha_{5} H_{13}^{\mu \alpha} H_{23}^{\rho \beta} V_{1}^{\nu} V_{2}^{\sigma}+\alpha_{6} H_{12}^{\mu \rho} H_{12}^{\nu \sigma} V_{3}^{\alpha} V_{3}^{\beta} \\
& +\alpha_{7}\left(H_{13}^{\mu \alpha} H_{13}^{\nu \beta} V_{2}^{\rho} V_{2}^{\sigma}+H_{23}^{\rho \alpha} H_{23}^{\sigma \beta} V_{1}^{\mu} V_{1}^{\nu}\right)+\alpha_{8} H_{12}^{\mu \rho} H_{13}^{\nu \alpha} H_{23}^{\sigma \beta} \\
& + \text { permutations - traces }] .
\end{aligned}
$$

There are 6 constraints from the conservation of the energy-momentum tensor, and therefore only two independent OPE coefficients. We choose them to be

$$
\lambda_{T T \mathcal{S}}^{(1)}=\alpha_{1}+2 \alpha_{2}+4 \alpha_{7}, \quad \lambda_{T T \mathcal{S}}^{(2)}=-5 \alpha_{1}-12 \alpha_{2}+\alpha_{3}-9 \alpha_{5}+4 \alpha_{7}-6 \alpha_{8} .
$$

With this choice, the coefficients $\alpha_{i}$ are (specializing to $d=4$ )

$$
\begin{aligned}
& \alpha_{1}=-\frac{4\left(\Delta^{2}-29 \Delta-24\right) \lambda_{T T \mathcal{S}}^{(1)}+3 \Delta(\Delta-8) \lambda_{T T \mathcal{S}}^{(2)},}{2(\Delta+2)(\Delta+4)} \\
& \alpha_{2}=\frac{2\left(5 \Delta^{2}-78 \Delta-24\right) \lambda_{T T \mathcal{S}}^{(1)}+(\Delta-8)(5 \Delta-2) \lambda_{T T \mathcal{S}}^{(2)}}{4(\Delta+2)(\Delta+4)}, \\
& \alpha_{3}=-\frac{2\left(\Delta^{2}-114 \Delta+184\right) \lambda_{T T \mathcal{S}}^{(1)}+(\Delta-8)(7 \Delta-10) \lambda_{T T \mathcal{S}}^{(2)},}{4(\Delta+2)(\Delta+4)} \\
& \alpha_{4}=\frac{2\left(3 \Delta^{2}-54 \Delta+88\right) \lambda_{T T \mathcal{S}}^{(1)}+3(\Delta-2)(\Delta-8) \lambda_{T T \mathcal{S}}^{(2)}}{4(\Delta+2)(\Delta+4)}, \\
& \alpha_{5}=-\frac{2 \Delta(3 \Delta-32) \lambda_{T T \mathcal{S}}^{(1)}+\left(3 \Delta^{2}-20 \Delta+8\right) \lambda_{T T \mathcal{S}}^{(2)},}{4(\Delta+2)(\Delta+4)} \\
& \alpha_{6}=\frac{2\left(\Delta^{2}+17 \Delta-56\right) \lambda_{T T \mathcal{S}}^{(1)}-(\Delta-8)(2 \Delta-5) \lambda_{T T \mathcal{S}}^{(2)},}{4(\Delta+2)(\Delta+4)} \\
& \alpha_{7}=-\frac{2\left(\Delta^{2}-13 \Delta+8\right) \lambda_{T T \mathcal{S}}^{(1)}+(\Delta-1)(\Delta-8) \lambda_{T T \mathcal{S}}^{(2)},}{4(\Delta+2)(\Delta+4)} \\
& \alpha_{8}=-\frac{2\left(3 \Delta^{2}-39 \Delta+68\right) \lambda_{T T \mathcal{S}}^{(1)}+\left(3 \Delta^{2}-27 \Delta+44\right) \lambda_{T T \mathcal{S}}^{(2)}}{4(\Delta+2)(\Delta+4)} .
\end{aligned}
$$

The definition eq. (B.19) was chosen so that all the coefficients $\alpha_{i}$ are finite for all $\Delta$ allowed by unitarity (as well as $\Delta \rightarrow \infty$ ), as long as $\lambda_{T T \mathcal{S}}^{(1,2)}$ are finite.

Eqs. (B.20) are not valid for the case where $\mathcal{S}$ is the energy-momentum tensor, corresponding to $\Delta=d=4$. In this case there are algebraic degeneracies in the constraints above, and we only have 5 independent constraints. This is in agreement with the wellknown fact that there are 3 independent TTT OPE coefficients in $d=4$. Using this approach is cumbersome for this case because of the existence of contact terms, as discussed in section 2.4. We will instead use the Ward identities in momentum space to fix the contribution of the energy-momentum tensor. 


\section{B.3 Fourier transform of scalar correlators}

For scalar correlators, the Fourier transform into momentum space can be performed straightforwardly. We have

$$
\langle\mathcal{O}|\tilde{\mathcal{O}}(k)| 0\rangle=\int d^{d} x \frac{e^{i k \cdot x}}{\left(x_{\mathrm{W}}^{2}\right)^{\Delta}} \equiv \Pi_{\mathcal{O}}(k) \theta\left(k^{0}\right) \theta\left(-k^{2}\right),
$$

where

$$
\Pi_{\mathcal{O}}(k)=\frac{2^{d-2 \Delta+1} \pi^{(d+2) / 2}}{\Gamma(\Delta) \Gamma\left(\Delta-\frac{d-2}{2}\right)}\left(-k^{2}\right)^{\Delta-d / 2} .
$$

For the three-point function, we consider three scalar operators $\mathcal{O}_{i}$ with scaling dimensions $\Delta_{i}$, for which we obtain

$$
\begin{aligned}
\left\langle\mathcal{O}_{3}\left|\mathrm{~T}\left[\tilde{\mathcal{O}}_{1}\left(p_{1}\right) \tilde{\mathcal{O}}_{2}\left(p_{2}\right)\right]\right| 0\right\rangle & =\int d^{d} x_{1} d^{d} x_{2} \frac{e^{i\left(p_{1} \cdot x_{1}+p_{2} \cdot x_{2}\right)} \lambda_{\mathcal{O}_{1} \mathcal{O}_{2} \mathcal{O}_{3}}}{\left(x_{1, \mathrm{~W}}^{2}\right)^{\Delta_{31,2} / 2}\left(x_{2, \mathrm{~W}}^{2}\right)^{\Delta_{32,1} / 2}\left(x_{12, \mathrm{~F}}^{2}\right)^{\Delta_{12,3} / 2}} \\
& \equiv \lambda_{\mathcal{O}_{1} \mathcal{O}_{2} \mathcal{O}_{3}} \mathcal{F}\left(\Delta_{31,2}, \Delta_{32,1} ; \Delta_{12,3}\right)
\end{aligned}
$$

where $\Delta_{i j, k}=\left(\Delta_{i}+\Delta_{j}-\Delta_{k}\right) / 2$. We have restored the $i \epsilon$ here, using eq. (B.4) and defining

$$
x_{\mathrm{F}}^{2} \equiv x^{2}+i \epsilon .
$$

The function $\mathcal{F}$ is a function symmetric in its first two arguments, given by

$$
\mathcal{F}\left(\Delta_{1}, \Delta_{2} ; \Delta_{3}\right)=\frac{-2 i \pi^{d+1} \Gamma\left(\Delta_{1}+\Delta_{3}-\frac{d}{2}\right) \Gamma\left(\Delta_{2}+\Delta_{3}-\frac{d}{2}\right) \Gamma\left(\frac{d}{2}-\Delta_{3}\right)}{\Gamma\left(\Delta_{1}\right) \Gamma\left(\Delta_{2}\right) \Gamma\left(\Delta_{3}\right) \Gamma\left(\Delta_{\text {tot }}-\frac{d}{2}\right) \Gamma\left(\Delta_{\text {tot }}-d+1\right)}\left(\frac{s}{4}\right)^{\Delta_{\text {tot }}-d},
$$

where $\Delta_{\text {tot }}=\Delta_{1}+\Delta_{2}+\Delta_{3}$. Note that the result can be divergent, for example if $\Delta_{3}=$ $d / 2+n($ with $n \in \mathbb{N})$.

\section{B.4 Conformal Ward identities}

We use conformal Ward identities to determine the 2- and 3-point functions of general tensor operators in momentum space, eq. (B.1). The conformal generators act in momentum space as

$$
\begin{aligned}
{\left[P_{\mu}, \tilde{\mathcal{O}}(p)\right] } & =p_{\mu} \tilde{\mathcal{O}}(p), \\
{[D, \tilde{\mathcal{O}}(p)] } & =-i\left(-p^{\nu} \frac{\partial}{\partial p^{\nu}}+\Delta-d\right) \tilde{\mathcal{O}}(p), \\
{\left[M_{\mu \nu}, \tilde{\mathcal{O}}(p)\right]=} & -i\left(p_{\mu} \frac{\partial}{\partial p^{\nu}}-p_{\nu} \frac{\partial}{\partial p^{\mu}}+\Sigma_{\mu \nu}\right) \tilde{\mathcal{O}}(p), \\
{\left[K_{\mu}, \tilde{\mathcal{O}}(p)\right]=} & \left(-2 p^{\nu} \frac{\partial^{2}}{\partial p^{\mu} \partial p^{\nu}}+p_{\mu} \frac{\partial^{2}}{\partial p_{\nu} \partial p^{\nu}}\right. \\
& \left.+2(\Delta-d) \frac{\partial}{\partial p^{\mu}}+2 \Sigma_{\mu \nu} \frac{\partial}{\partial p_{\nu}}\right) \tilde{\mathcal{O}}(p),
\end{aligned}
$$


where $\Sigma_{\mu \nu}$ is the spin operator, acting on a spin- $\ell$ tensor as

$$
\Sigma_{\mu \nu} \tilde{\mathcal{O}}^{\alpha_{1} \ldots \alpha_{\ell}}=\sum_{i=1}^{\ell}\left(\delta_{\mu}^{\alpha_{i}} \tilde{\mathcal{O}}^{\alpha_{1} \ldots \ldots \alpha_{\ell}}-\delta_{\nu}^{\alpha_{i}} \tilde{\mathcal{O}}_{\mu}^{\alpha_{1} \ldots \ldots \alpha_{\ell}}\right)
$$

The states $\left|\mathcal{O}^{\alpha_{1} \cdots \alpha_{\ell}}\right\rangle$ that appear in the matrix elements eq. (B.1) transform as ${ }^{15}$

$$
\begin{aligned}
D\left|\mathcal{O}^{\alpha_{1} \ldots \alpha_{\ell}}\right\rangle & =-i \Delta\left|\mathcal{O}^{\alpha_{1} \ldots \alpha_{\ell}}\right\rangle, \\
M_{\mu \nu}\left|\mathcal{O}^{\alpha_{1} \ldots \alpha_{\ell}}\right\rangle & =-i \Sigma_{\mu \nu}\left|\mathcal{O}^{\alpha_{1} \ldots \alpha_{\ell}}\right\rangle, \\
K_{\mu}\left|\mathcal{O}^{\alpha_{1} \ldots \alpha_{\ell}}\right\rangle & =0 .
\end{aligned}
$$

Conformal Ward identities follow then from invariance of the vacuum state, which in turns implies, e.g. for special conformal transformations,

$$
\left\langle\mathcal{O}^{\alpha_{1} \ldots \alpha_{\ell}}\left|\left[K_{\tau}, \tilde{T}^{\mu \nu}\left(p_{1}\right)\right] \tilde{T}^{\rho \sigma}\left(p_{2}\right)\right| 0\right\rangle+\left\langle\mathcal{O}^{\alpha_{1} \ldots \alpha_{\ell}}\left|\tilde{T}^{\mu \nu}\left(p_{1}\right)\left[K_{\tau}, \tilde{T}^{\rho \sigma}\left(p_{2}\right)\right]\right| 0\right\rangle=0
$$

This Ward identity is satisfied in the above form where there is no time ordering of the momentum-space operators. In this case, there are no UV divergences or contact terms as discussed in section 2.3 and section 2.4. (The absence of UV divergences also implies the absence of anomalies.) When considering time-ordered correlators, we have to worry about these subtleties. However, the only case where there are contact terms is when $\mathcal{O}$ is the energy-momentum tensor itself. There are no conformal anomalies in these correlation functions, because conformal anomalies are purely local and therefore do not contribute to correlation functions where some operators are Wightman ordered. Our correlator (B.1) therefore obeys non-anomalous Ward identities with no additional contact terms like eq. (B.31) above, namely

$$
\begin{aligned}
& \sum_{i=1}^{2}\left[-2 p_{i}^{\omega} \frac{\partial^{2}}{\partial p_{i}^{\tau} \partial p_{i}^{\omega}}+p_{i \tau} \frac{\partial^{2}}{\partial p_{i \omega} \partial p_{i}^{\omega}}+2 \Sigma_{\tau \omega}^{(i)} \frac{\partial}{\partial p_{i \omega}}\right] \\
& \times\left\langle\mathcal{O}^{\alpha_{1} \ldots \alpha_{\ell}}\left|\mathrm{T}\left[T^{\mu \nu}\left(p_{1}\right) T^{\rho \sigma}\left(p_{2}\right)\right]\right| 0\right\rangle=0
\end{aligned}
$$

where the $\Sigma_{\tau \omega}^{(i)}$ only acts on the indices of the operator carrying momentum $p_{i}$. This equation is quite non-trivial to solve, and its discussion is postponed to section B.6.

In the case of Wightman two-point functions, conformal invariance is simple enough

\footnotetext{
${ }^{15}$ These states correspond to inserting a position-space operator at $x=0$, and the resulting momentumspace correlation functions have the momentum-conserving delta function factored out. An alternative approach is to work with the full momentum space correlation function, which includes the momentumconserving delta function. In this case, the differential operators must act on the delta function. This approach is discussed in ref. [24].
} 
to allow for a direct solution. For our traceless symmetric spin-two operator $\mathcal{S}$, we obtain

$$
\begin{aligned}
{\left[\Pi_{\mathcal{S}}(k)\right]^{\mu \nu \rho \sigma}=} & \tilde{C}_{\mathcal{S}}\left[\frac{1}{2}\left(\eta^{\mu \rho} \eta^{\nu \sigma}+\eta^{\mu \sigma} \eta^{\nu \rho}\right)-\frac{\Delta(\Delta+1)-d}{\Delta(\Delta-1) d} \eta^{\mu \nu} \eta^{\rho \sigma}\right. \\
& -\frac{2 \Delta-d}{2 \Delta}\left(\eta^{\mu \rho} \frac{k^{\nu} k^{\sigma}}{k^{2}}+\eta^{\mu \sigma} \frac{k^{\nu} k^{\rho}}{k^{2}}+\eta^{\nu \rho} \frac{k^{\mu} k^{\sigma}}{k^{2}}+\eta^{\nu \sigma} \frac{k^{\mu} k^{\rho}}{k^{2}}\right) \\
& +\frac{2 \Delta-d}{\Delta(\Delta-1)}\left(\eta^{\mu \nu} \frac{k^{\rho} k^{\sigma}}{k^{2}}+\eta^{\rho \sigma} \frac{k^{\mu} k^{\nu}}{k^{2}}\right) \\
& \left.+\frac{(2 \Delta-d)(2 \Delta-d-2)}{\Delta(\Delta-1)} \frac{k^{\mu} k^{\nu} k^{\rho} k^{\sigma}}{\left(k^{2}\right)^{2}}\right] s^{\Delta-d / 2},
\end{aligned}
$$

where $k=p_{1}+p_{2}$, and $\tilde{C}_{\mathcal{S}}$ is a coefficient that is not fixed by conformal invariance. The inverse of this tensor is the object that enters in the completeness relation eq. (2.1) and thus in the computation of the conformal blocks. It is given by

$$
\begin{aligned}
{\left[\Pi_{\mathcal{S}}^{-1}(k)\right]^{\mu \nu \rho \sigma}=} & \frac{s^{d / 2-\Delta}}{\tilde{C}_{\mathcal{S}}}\left[\frac{1}{2}\left(\eta^{\mu \rho} \eta^{\nu \sigma}+\eta^{\mu \sigma} \eta^{\nu \rho}\right)\right. \\
& -\frac{2 \Delta-d}{2(\Delta-d)}\left(\eta^{\mu \rho} \frac{k^{\nu} k^{\sigma}}{k^{2}}+\eta^{\mu \sigma} \frac{k^{\nu} k^{\rho}}{k^{2}}+\eta^{\nu \rho} \frac{k^{\mu} k^{\sigma}}{k^{2}}+\eta^{\nu \sigma} \frac{k^{\mu} k^{\rho}}{k^{2}}\right) \\
& \left.+\frac{(2 \Delta-d)(2 \Delta-d+2)}{(\Delta-d)(\Delta-d+1)} \frac{k^{\mu} k^{\nu} k^{\rho} k^{\sigma}}{\left(k^{2}\right)^{2}}\right] .
\end{aligned}
$$

Note that the expression for $\Pi_{\mathcal{S}}$ also holds in the case of the energy-momentum tensor by setting $\Delta=d$, or in a simpler form

$$
\begin{aligned}
{\left[\Pi_{T}(k)\right]^{\mu \nu \rho \sigma}=} & \tilde{C}_{T} s^{d / 2}\left[\frac{1}{2}\left(\tilde{\mathcal{I}}^{\mu \rho}(k) \tilde{\mathcal{I}}^{\nu \sigma}(k)+\tilde{\mathcal{I}}^{\mu \sigma}(k) \tilde{\mathcal{I}}^{\nu \rho}(k)\right)\right. \\
& \left.-\frac{1}{d-1} \tilde{\mathcal{I}}^{\mu \nu}(k) \tilde{\mathcal{I}}^{\rho \sigma}(k)\right]
\end{aligned}
$$

where $\tilde{C}_{T}=4 \pi c$ and

$$
\tilde{\mathcal{I}}^{\mu \nu}(k)=\eta^{\mu \nu}-\frac{k^{\mu} k^{\nu}}{k^{2}} .
$$

The inverse of eq. (B.34) appears to be ill-defined in the limit $\Delta \rightarrow d$. This is the case of the conserved energy-momentum tensor, and its inverse is not unique because it is transverse, $k_{\mu} \tilde{T}^{\mu \nu}(k)=0$. We can invert it on the transverse space, since transverse contributions vanish by eq. (B.42b). We therefore obtain

$$
\left[\Pi_{T}^{-1}(k)\right]^{\mu \nu \rho \sigma}=\frac{1}{\tilde{C}_{T} s^{d / 2}} \frac{1}{2}\left(\eta^{\mu \rho} \eta^{\nu \sigma}+\eta^{\mu \sigma} \eta^{\nu \rho}\right) .
$$

\section{B.5 Conservation Ward identities}

In addition to the conformal Ward identities, there are identities encoding the fact that the energy-momentum tensor is a traceless, conserved current, i.e. that it belongs into a short representation of the conformal algebra. The precise form of these Ward identities 
depend on our definition of the energy-momentum tensor. With our choice (1.1) the Ward identities are summarized by [25]

$$
\begin{aligned}
\nabla_{\mu}\left\langle 0\left|T^{\mu \nu}(x)\right| 0\right\rangle_{g} & =0, \\
g_{\mu \nu}\left\langle 0\left|T^{\mu \nu}(x)\right| 0\right\rangle_{g} & =c W^{\mu \nu \rho \sigma} W_{\mu \nu \rho \sigma}+a E_{4},
\end{aligned}
$$

with $\nabla_{\mu}$ the covariant derivative in the metric $g_{\mu \nu}$. The Ward identities for higher correlation functions follow by repeated differentiation with respect to the metric $g_{\mu \nu}$ or other source fields.

For example, the trace Ward identity eq. (B.39) gives in position space

$$
\begin{aligned}
\eta_{\mu \nu}\left\langle 0\left|T^{\alpha \beta}(z) \mathrm{T}\left[T^{\mu \nu}\left(x_{1}\right) T^{\rho \sigma}\left(x_{2}\right)\right]\right| 0\right\rangle & =2 \delta^{4}\left(x_{1}-x_{2}\right)\left\langle 0\left|T^{\alpha \beta}(z) T^{\rho \sigma}\left(x_{2}\right)\right| 0\right\rangle, \\
\eta_{\alpha \beta}\left\langle 0\left|T^{\alpha \beta}(z) \mathrm{T}\left[T^{\mu \nu}\left(x_{1}\right) T^{\rho \sigma}\left(x_{2}\right)\right]\right| 0\right\rangle & =0, \\
\eta_{\mu \nu}\left\langle 0\left|\mathcal{O}^{\alpha_{1} \ldots \alpha_{\ell}}(z) \mathrm{T}\left[T^{\mu \nu}\left(x_{1}\right) T^{\rho \sigma}\left(x_{2}\right)\right]\right| 0\right\rangle & =0 . \quad(\mathcal{O} \neq T)
\end{aligned}
$$

Note that the anomaly terms on the right-hand side of eq. (B.39) do not contribute, because they are $O\left(h_{\mu \nu}^{2}\right)$, and therefore only appear if there are 3 or more energy-momentum tensors in the same time ordered product.

In momentum space, these become

$$
\begin{aligned}
\eta_{\mu \nu}\left\langle T^{\alpha \beta}\left|\mathrm{T}\left[\tilde{T}^{\mu \nu}\left(p_{1}\right) \tilde{T}^{\rho \sigma}\left(p_{2}\right)\right]\right| 0\right\rangle & =2\left\langle T^{\alpha \beta}\left|\tilde{T}^{\rho \sigma}\left(p_{1}+p_{2}\right)\right| 0\right\rangle, \\
\eta_{\alpha \beta}\left\langle T^{\alpha \beta}\left|\mathrm{T}\left[\tilde{T}^{\mu \nu}\left(p_{1}\right) \tilde{T}^{\rho \sigma}\left(p_{2}\right)\right]\right| 0\right\rangle & =0 . \\
\eta_{\mu \nu}\left\langle\mathcal{O}^{\alpha_{1} \ldots \alpha_{\ell}}\left|\mathrm{T}\left[\tilde{T}^{\mu \nu}\left(p_{1}\right) \tilde{T}^{\rho \sigma}\left(p_{2}\right)\right]\right| 0\right\rangle & =0 . \quad(\mathcal{O} \neq T)
\end{aligned}
$$

Similar Ward identities can be derived for the divergence of the energy-momentum tensor using eq. (B.38):

$$
\begin{aligned}
\left(p_{1}\right)_{\mu}\left\langle T^{\alpha \beta}\left|\mathrm{T}\left[\tilde{T}^{\mu \nu}\left(p_{1}\right) \tilde{T}^{\rho \sigma}\left(p_{2}\right)\right]\right| 0\right\rangle= & \left(p_{2}\right)^{\nu}\left\langle T^{\alpha \beta}\left|\tilde{T}^{\rho \sigma}\left(p_{1}+p_{2}\right)\right| 0\right\rangle \\
& -\left(p_{2}\right)_{\lambda} \eta^{\nu \rho}\left\langle T^{\alpha \beta}\left|\tilde{T}^{\sigma \lambda}\left(p_{1}+p_{2}\right)\right| 0\right\rangle \\
& -\left(p_{2}\right)_{\lambda} \eta^{\nu \sigma}\left\langle T^{\alpha \beta}\left|\tilde{T}^{\rho \lambda}\left(p_{1}+p_{2}\right)\right| 0\right\rangle . \\
\left(p_{1}+p_{2}\right)_{\alpha}\left\langle T^{\alpha \beta}\left|\mathrm{T}\left[\tilde{T}^{\mu \nu}\left(p_{1}\right) \tilde{T}^{\rho \sigma}\left(p_{2}\right)\right]\right| 0\right\rangle= & 0, \\
\left(p_{1}\right)_{\mu}\left\langle\mathcal{O}^{\alpha_{1} \ldots \alpha_{\ell}}\left|\mathrm{T}\left[\tilde{T}^{\mu \nu}\left(p_{1}\right) \tilde{T}^{\rho \sigma}\left(p_{2}\right)\right]\right| 0\right\rangle= & 0 . \quad(\mathcal{O} \neq T)
\end{aligned}
$$

Some of these identities involve the Wightman 2-point function given by eq. (B.35) on the right-hand side. Note that Ward identities for momentum-space 3-point functions have been obtained in refs. [26, 27]; they differ from ours, due to our specific ordering of operators.

\section{B.6 Using Ward identities to compute 3-point functions}

We now show how to use the Ward identities listed above to determine the 3-point functions eq. (3.6) up to OPE coefficients. This is greatly simplified by the fact that we need the result only for massless external momenta.

The Ward identities for translation, Lorentz, and dilatation symmetry can be solved simply by writing the 3-point function in terms of appropriate invariants. Translation 
invariance implies that the 3 -point function is a function of only two momenta $p_{1,2}$. Lorentz invariance requires that it is a Lorentz tensor made from $p_{1,2}^{\mu}$ and $\eta^{\mu \nu}$. Dilatation invariance implies that this tensor has the appropriate dimension.

The only remaining Ward identity is the one for special conformal transformations, which must be solved explicity. Although we are interested in the 3-point function for $p_{1,2}^{2}=0$, we must consider $p_{1,2}^{2} \neq 0$ because the special conformal Ward identity relates configurations with different values of $p_{1,2}^{2}$. We are interested in kinematics with $s=$ $-\left(p_{1}+p_{2}\right)^{2}>0$, so we can write the correlator in terms of the dimensionless quantities

$$
\hat{p}_{1}^{\mu}=\frac{p_{1}^{\mu}}{\sqrt{s}}, \quad \hat{p}_{2}^{\mu}=\frac{p_{2}^{\mu}}{\sqrt{s}}, \quad \hat{m}_{1}^{2}=\frac{p_{1}^{2}}{s}, \quad \hat{m}_{2}^{2}=\frac{p_{2}^{2}}{s} .
$$

The integral (3.6) can then be parametrized as

$$
\begin{aligned}
& \left\langle\mathcal{O}^{\alpha_{1} \ldots \alpha_{\ell}}\left|\mathrm{T}\left[\tilde{T}^{\mu \nu}\left(p_{1}\right) \tilde{T}^{\rho \sigma}\left(p_{2}\right)\right]\right| 0\right\rangle \\
& \quad=s^{\Delta / 2} \sum_{a=1}^{n} h_{a}\left(\hat{m}_{1}^{2}, \hat{m}_{2}^{2}\right) t_{a}^{(\mu \nu)(\rho \sigma) \alpha_{1} \ldots \alpha_{\ell}}\left(\hat{p}_{1}^{\mu}, \hat{p}_{2}^{\mu}, \eta^{\mu \nu}\right),
\end{aligned}
$$

where the $h_{a}$ are arbitrary functions of the two variables $\hat{m}_{1}^{2}$ and $\hat{m}_{2}^{2}$, and the $t_{a}$ are all possible tensors constructed from the dimensionless quantities in eq. (B.43). Because we eventually want to take $p_{1}^{2}=p_{2}^{2} \rightarrow 0$, we only need to know the $h_{a}(0,0)$. However, the special conformal Ward identity eq. (B.32) relates the $h_{a}(0,0)$ with derivatives $\partial_{\hat{m}_{i}^{2}} h_{a}(0,0)$. Explicitly, the Ward identity becomes

$$
\begin{aligned}
& \sum_{a=1}^{n} \sum_{i=1}^{2}\left[2 s^{\Delta / 2-1} \partial_{\hat{m}_{i}^{2}} h_{a}(0,0)\left((d-2) p_{i \tau}+2 p_{i}^{\lambda} \Sigma_{\tau \lambda}^{(i)}\right) t_{a}^{(\mu \nu)(\rho \sigma) \alpha_{1} \ldots \alpha_{\ell}}\right. \\
& \left.+h_{a}(0,0)\left(-2 p_{i}^{\lambda} \frac{\partial^{2}}{\partial p_{i}^{\tau} \partial p_{i}^{\lambda}}+p_{i \tau} \frac{\partial^{2}}{\partial p_{i \lambda} \partial p_{i}^{\lambda}}+2 \Sigma_{\tau \lambda}^{(i)} \frac{\partial}{\partial p_{i \lambda}}\right)\left(s^{\Delta / 2} t_{a}^{(\mu \nu)(\rho \sigma) \alpha_{1} \ldots \alpha_{\ell}}\right)\right]=0 .
\end{aligned}
$$

There are no second derivative terms of the form $\partial_{\hat{m}_{i}^{2}} \partial_{\hat{m}_{i}^{2}} h_{a}(0,0) \cdot{ }^{16}$ Eq. (B.45) provides a set of linear constraints involving both $\partial_{\hat{m}_{i}^{2}} h_{a}(0,0)$ and $h_{a}(0,0)$. But we can eliminate $\partial_{\hat{m}_{i}^{2}} h_{a}(0,0)$ and obtain a set of constraints among $h_{a}(0,0)$ themselves. To do this, we note that contracting eq. (B.45) with $p_{2}^{\tau}$, the terms involving $\partial_{\hat{m}_{2}^{2}} h_{a}(0,0)$ vanish, giving us a relation between $\partial_{\hat{m}_{1}^{2}} h_{a}(0,0)$ and $h_{a}(0,0)$. The tensor multiplying $\partial_{\hat{m}_{1}^{2}} h_{a}(0,0)$ in this expression is invertible and the resulting linear equations have a unique solution. Similarly, contracting with $p_{1}^{\tau}$ allows us to fix $\partial_{\hat{m}_{2}^{2}} h_{a}(0,0)$. The equations are rather complicated, but are straightforward to solve using computer algebra. We will give several explicit examples below.

Scalar states. In the case where $\mathcal{O}$ is a scalar operator, the most general tensor $t^{(\mu \nu)(\rho \sigma)}$ built out of $\hat{p}_{1,2}^{\mu}$ and $\eta^{\mu \nu}$ that is symmetric in both pairs of indices has 21 independent tensor structures. After imposing the special conformal Ward identity and considering on-shell momenta $p_{1,2}^{2}=0$, only 6 linearly independent tensor structures remain, of which

\footnotetext{
${ }^{16}$ This is because the differential operator in eq. (B.32) is related to the Todorov operator that preserves the condition $p_{i}^{2}=0[28,29]$.
} 
5 are symmetric under the exchange of the two energy-momentum tensors. Imposing the transversality and trace conditions eqs. (B.41c) and (B.42c), only one unique linear combination of the 5 previous structures remains. It can be written as

$$
\begin{aligned}
\langle\mathcal{O}| \mathrm{T} & {\left[\tilde{T}^{\mu \nu}\left(p_{1}\right) \tilde{T}^{\rho \sigma}\left(p_{2}\right)\right]|0\rangle } \\
= & \tilde{\lambda}_{T T \mathcal{O}} s^{\Delta / 2}\left\{\left(\eta^{\mu \nu}+2 \hat{p}_{1}^{\mu} \hat{p}_{2}^{\nu}+2 \hat{p}_{2}^{\mu} \hat{p}_{1}^{\nu}\right)\left(\eta^{\rho \sigma}+2 \hat{p}_{1}^{\rho} \hat{p}_{2}^{\sigma}+2 \hat{p}_{2}^{\rho} \hat{p}_{1}^{\sigma}\right)\right. \\
& -\frac{d-2}{2}\left[\left(\eta^{\mu \rho}+2 \hat{p}_{2}^{\mu} \hat{p}_{1}^{\rho}-\xi_{1} \hat{p}_{1}^{\mu} \hat{p}_{2}^{\rho}\right)\left(\eta^{\nu \sigma}+2 \hat{p}_{2}^{\nu} \hat{p}_{1}^{\sigma}-\xi_{1} \hat{p}_{1}^{\nu} \hat{p}_{2}^{\sigma}\right)\right. \\
& \left.+(\rho \leftrightarrow \sigma)]+\xi_{2} \hat{p}_{1}^{\mu} \hat{p}_{1}^{\nu} \hat{p}_{2}^{\rho} \hat{p}_{2}^{\sigma}\right\} .
\end{aligned}
$$

This is a polynomial in the momenta because we are evaluating the correlation function for $p_{1,2}^{2}=0$; for general momenta it would be a much more complicated function. The numerical factors $\xi_{1}$ and $\xi_{2}$ are given by

$$
\begin{aligned}
\xi_{1}= & \frac{\Delta(\Delta-d)+2(d-6)}{(d-2)^{2}} \\
\xi_{2}= & \frac{1}{(d-1)(d-2)^{3}(d-4)^{2}}\left[\left(3 d^{2}-12 d+8\right) \Delta^{2}(\Delta-d)^{2}\right. \\
& +2\left(d^{4}-2 d^{3}-44 d^{2}+160 d-112\right) \Delta(\Delta-d) \\
& \left.\left.+4(d-1)(d-4)(d-6)\left(3 d^{2}-6 d-8\right)\right)\right]
\end{aligned}
$$

Note that $\xi_{2}$ diverges as $d \rightarrow 4$. However, this multiplies a tensor structure that vanishes when we contract with transverse polarization tensors, and therefore does not affect our results. Also note that eq. (B.46) is invariant under the symmetry $\Delta \rightarrow d-\Delta$ up to the overall scale factor. This is not an accident; it reflects the fact that for each operator $\mathcal{O}$, there exist an non-local "shadow" operator with scaling dimension $d-\Delta$ that satisfies the same Ward identities as $\mathcal{O}[30,31]$.

For the physical graviton polarizations in $d=4$, we obtain

$$
\begin{aligned}
& \left\langle\mathcal{O}\left|\mathrm{T}\left[\tilde{T}_{+}\left(p_{1}\right) \tilde{T}_{+}\left(p_{2}\right)\right]\right| 0\right\rangle=2 \tilde{\lambda}_{T T \mathcal{O}} s^{\Delta / 2}, \\
& \left\langle\mathcal{O}\left|\mathrm{T}\left[\tilde{T}_{+}\left(p_{1}\right) \tilde{T}_{-}\left(p_{2}\right)\right]\right| 0\right\rangle=0 .
\end{aligned}
$$

The vanishing of the 3-point function for +- initial helicity can be understood from angular momentum conservation. For the ++ initial helicity, the conformal block can then be directly computed from the square of the three-point function, weighted with the inverse of $\Pi_{\mathcal{O}}\left(p_{1}+p_{2}\right)$ given in eq. (B.22), to get

$$
\lambda_{T T \mathcal{O}}^{2} f(\mathcal{O})=\lambda_{T T \mathcal{O}}^{2} \frac{5}{2} f^{(+)}(\mathcal{O})=\left|\tilde{\lambda}_{T T \mathcal{O}}\right|^{2} \frac{2^{2 \Delta-5} \Gamma(\Delta) \Gamma(\Delta-1)}{\pi^{4}} .
$$

The coefficient $\tilde{\lambda}_{T T \mathcal{O}}$ is arbitrary. It will be related to the position-space OPE coefficient in the following section. 
Spin-2 states. We now consider states created by the 2-index traceless symmetric tensor $\mathcal{S}$. Imposing symmetry in each pair of indices as well as under the exchange of the two time-ordered operators leads to 77 structures. The Ward identity for special conformal transformations brings this number down to 17 tensor structures. Imposing the transversality and trace conditions eqs. (B.41a)-(B.42c) reduces this number further to only 2 structures.

If we only consider the transverse and traceless part of this three-point function, i.e. if we ignore all terms proportional to $p_{1}^{\mu}, p_{1}^{\nu}, p_{2}^{\rho}, p_{2}^{\sigma},\left(p_{1}+p_{2}\right)^{\alpha},\left(p_{1}+p_{2}\right)^{\beta}$, as well as $\eta^{\mu \nu}, \eta^{\rho \sigma}$ or $\eta^{\alpha \beta}$, then the result can be written

$$
\begin{aligned}
\left\langle\mathcal{S}^{\alpha \beta}\left|\mathrm{T}\left[\tilde{T}^{\mu \nu}\left(p_{1}\right) \tilde{T}^{\rho \sigma}\left(p_{2}\right)\right]\right| 0\right\rangle= & s^{\Delta / 2}\left[\tilde{\lambda}_{T T \mathcal{S}}^{(1)} t_{1}^{(\mu \nu)(\rho \sigma)(\alpha \beta)}+\tilde{\lambda}_{T T \mathcal{S}}^{(2)} t_{2}^{(\mu \nu)(\rho \sigma)(\alpha \beta)}\right. \\
& +(\text { longitudinal/trace parts })]
\end{aligned}
$$

where

$$
\begin{aligned}
& t_{1}^{\mu \nu \rho \sigma \alpha \beta}=\left[\eta^{\mu \rho}+2 \hat{p}_{2}^{\mu} \hat{p}_{1}^{\rho}\right]\left[\eta^{\nu \sigma}+2 \hat{p}_{2}^{\nu} \hat{p}_{1}^{\sigma}\right]\left(\hat{p}_{1}-\hat{p}_{2}\right)^{\alpha}\left(\hat{p}_{1}-\hat{p}_{2}\right)^{\beta}, \\
& t_{2}^{\mu \nu \rho \sigma \alpha \beta}=\left[\eta^{\mu \rho}+2 \hat{p}_{2}^{\mu} \hat{p}_{1}^{\rho}\right]\left[\eta^{\nu \alpha}+\hat{p}_{2}^{\nu}\left(\hat{p}_{1}-\hat{p}_{2}\right)^{\alpha}\right]\left[\eta^{\sigma \beta}-\hat{p}_{1}^{\sigma}\left(\hat{p}_{1}-\hat{p}_{2}\right)^{\beta}\right],
\end{aligned}
$$

and symmetrization in each pair of indices is understood in eq. (B.52). Again, the number of tensor structures (2) is in agreement with the position-space result of section B.2.

This result can be used to compute the conformal blocks in terms of the momentumspace OPE coefficients $\tilde{\lambda}_{T T \mathcal{S}}^{(1,2)}$, and we get (in $d=4$ )

$$
\begin{aligned}
\sum_{a, b=1}^{2} \lambda_{T T \mathcal{S}}^{(a)} \lambda_{T T \mathcal{S}}^{(b)} f_{a b}(\mathcal{S})= & \sum_{a, b=1}^{2} \tilde{\lambda}_{T T \mathcal{S}}^{(a)} \tilde{\lambda}_{T T \mathcal{S}}^{(b)} \frac{2^{2 \Delta-9}(\Delta+1) \Gamma(\Delta-1)^{2}}{\pi^{4}(\Delta-1)(\Delta-3)} \\
& \times\left(\begin{array}{cc}
-3 \Delta(\Delta-4)-8 & \Delta(\Delta-4)+4 \\
\Delta(\Delta-4)+4 & -\Delta(\Delta-4)-2
\end{array}\right)_{a b}
\end{aligned}
$$

Energy-momentum states. In the special case in which the spin-2 operator is the energy momentum tensor, the Ward identities are modified due to the presence of contact terms, eqs. (B.41a) and (B.42a). In addition to the two structures of eq. (B.52), the 3point function admits therefore a third tensor structure proportional to $\tilde{C}_{T}$ (and hence $c$ ) through its appearance in the two-point function of $T$, namely

$$
\begin{aligned}
\left\langle T^{\alpha \beta}\left|\mathrm{T}\left[\tilde{T}^{\mu \nu}\left(p_{1}\right) \tilde{T}^{\rho \sigma}\left(p_{2}\right)\right]\right| 0\right\rangle= & s^{2}\left[\tilde{\lambda}_{T T T}^{(1)} t_{1}^{(\mu \nu)(\rho \sigma)(\alpha \beta)}+\tilde{\lambda}_{T T T}^{(2)} t_{2}^{(\mu \nu)(\rho \sigma)(\alpha \beta)}\right. \\
& +\tilde{C}_{T} t_{c}^{(\mu \nu)(\rho \sigma)(\alpha \beta)} \\
& +(\text { longitudinal/trace parts })]
\end{aligned}
$$

where $t_{1}$ and $t_{2}$ are given in eqs. (B.53a) and (B.53b), while $t_{c}$ is given by

$$
\begin{aligned}
t_{c}^{\mu \nu \rho \sigma \alpha \beta}= & 2 \eta^{\mu \rho} \eta^{\nu \alpha} \eta^{\sigma \beta}+2 \eta^{\mu \rho}\left(\eta^{\nu \alpha} \hat{p}_{1}^{\sigma}-\eta^{\sigma \alpha} \hat{p}_{2}^{\nu}\right)\left(\hat{p}_{1}-\hat{p}_{2}\right)^{\beta} \\
& -2\left(\eta^{\mu \alpha} \eta^{\nu \beta} \hat{p}_{1}^{\rho} \hat{p}_{1}^{\sigma}+\eta^{\rho \alpha} \eta^{\sigma \beta} \hat{p}_{2}^{\mu} \hat{p}_{2}^{\nu}\right) \\
& +4 \eta^{\mu \alpha} \eta^{\rho \beta} \hat{p}_{2}^{\nu} \hat{p}_{1}^{\sigma}-\frac{1}{2} \eta^{\mu \rho} \eta^{\nu \sigma}\left(\hat{p}_{1}-\hat{p}_{2}\right)^{\alpha}\left(\hat{p}_{1}-\hat{p}_{2}\right)^{\beta} .
\end{aligned}
$$


Projecting these tensors onto physical graviton polarizations, we obtain ${ }^{17}$

$$
\begin{aligned}
\left\langle T^{\alpha \beta}\left|\mathrm{T}\left[\tilde{T}_{+}\left(p_{1}\right) \tilde{T}_{+}\left(p_{2}\right)\right]\right| 0\right\rangle= & \frac{1}{2}\left(\tilde{C}_{T}-\frac{2}{3} \tilde{\lambda}_{T T T}^{(1)}+\frac{1}{3} \tilde{\lambda}_{T T T}^{(2)}\right) s^{2} \\
& \times\left[\eta^{\alpha \beta}+4\left(\hat{p}_{1}^{\alpha} \hat{p}_{2}^{\beta}+\hat{p}_{2}^{\alpha} \hat{p}_{1}^{\beta}\right)-2\left(\hat{p}_{1}^{\alpha} \hat{p}_{1}^{\beta}+\hat{p}_{2}^{\alpha} \hat{p}_{2}^{\beta}\right)\right], \\
\left\langle T^{\alpha \beta}\left|\mathrm{T}\left[\tilde{T}_{+}\left(p_{1}\right) \tilde{T}_{-}\left(p_{2}\right)\right]\right| 0\right\rangle= & 0 .
\end{aligned}
$$

For general $d$, eq. (B.55) depends on 3 unknown constants, in agreement with the fact that there are 3 independent OPE coefficients in general $d$ [21]. However, in $d=4$ we find that only one linear combination of the OPE coefficients appears in these results. This is analogous to the vanishing of the momentum space OPE coefficients at special values of $\Delta$. Specifically, we find that in all 4D CFTs we have $\tilde{\lambda}_{T T T}^{(2)}=2 \tilde{\lambda}_{T T T}^{(1)}$. There are two ways to prove this. First, the contribution of a generic spin-2 operator vanishes in the limit $\Delta \rightarrow 4$. Since this corresponds to the eq. (B.55) with $\tilde{C}_{T}=0$ (compare eq. (B.52)), we conclude that the contribution from the energy-momentum tensor is proportional to $\tilde{C}_{T}=4 \pi c$. Alternatively, the two OPE coefficients $\tilde{\lambda}_{T T T}^{(1,2)}$ can be computed in each of the 3 free theories, and subsequently expressed in terms of $n_{\mathrm{s}}, n_{\mathrm{f}}$ and $n_{\mathrm{v}}$ (see section 4.5). In fact, we obtain

$$
\tilde{\lambda}_{T T T}^{(2)}=2 \tilde{\lambda}_{T T T}^{(1)}=4 \pi(c-a),
$$

where $a$ is the anomaly coefficient given in eq. (A.6).

The conformal block can therefore be directly computed as a function of $c$ only, using the inverse of the two-point function in eq. (B.37), and we obtain

$$
\left|\mathcal{M}_{T_{+} T_{+} \rightarrow T}\left(p_{1}, p_{2}\right)\right|^{2}=6 \pi c s^{2}, \quad\left|\mathcal{M}_{T_{+} T_{-} \rightarrow T}\left(p_{1}, p_{2}\right)\right|^{2}=0,
$$

or equivalently (see eq. (3.14))

$$
\sum_{a, b=1}^{3} \lambda_{T T T}^{(a)} \lambda_{T T T}^{(b)}\left[f_{a b}^{(+)}(T)+f_{a b}^{(-)}(T)\right]=\frac{3}{5} c .
$$

This agrees with the result of the free field theory calculation in section 4 .

\section{B.7 Relation to position space OPE coefficients}

In the previous section, we have presented a method that allows to evaluate the 3-point function (3.6) up to unknown momentum-space OPE coefficient, and it only remains to relate them with the definitions of section B.2, in order to obtain the conformal blocks for operators that are not the energy-momentum tensor itself.

${ }^{17}$ We have eliminated all dependence on polarization vectors on the right-hand side by use of the identity

$$
\epsilon_{+}^{(\mu}\left(p_{1} \mid p_{2}\right) \epsilon_{+}^{\nu)}\left(p_{2} \mid p_{1}\right)=e^{i \theta}\left(\frac{1}{2} \eta^{\mu \nu}-\frac{p_{1}^{(\mu} p_{2}^{\nu)}}{p_{1} \cdot p_{2}}\right),
$$

The phase $\theta$ can be thought of as an arbitrary phase in the definition of the polarization tensors, which cancels in our sum rule because it involves the square of the 3-point function. 
Scalar operator. We consider the following Lorentz scalar quantity

$$
Q(s)=\left[p_{2}^{\mu} p_{1}^{\rho}-\left(p_{1} \cdot p_{2}\right) \eta^{\mu \rho}\right] \eta^{\nu \sigma}\langle\mathcal{O}| \mathrm{T}\left[\tilde{T}_{\mu \nu}\left(p_{1}\right) \tilde{T}_{\rho \sigma}\left(p_{2}\right)|0\rangle .\right.
$$

The tensor in square brackets is transverse, so this is a well-defined object in $d \rightarrow 4$. On the one hand, from the result of the Ward identity analysis and in particular eq. (B.46), it must be equal to

$$
Q(s)=-\tilde{\lambda}_{T T \mathcal{O}} \frac{\Delta(\Delta-4)+20}{8} s^{(\Delta+2) / 2} .
$$

On the other hand, we can compute this same quantity by direct Fourier transform of eq. (B.12) from position to momentum space. Note that this Fourier transform in general is ambiguous due to contact terms, but as we have argued repeatedly above, there are no contact terms in this case. For generic values of the the dimension of $\mathcal{O}$, the Fourier transform is unambiguous, and for other values we can define the Fourier transform by analytic continuation. We expect subtleties only in the case of exactly marginal operators, as discussed in section 2.4. The integrals can be performed using integration by parts to relate all terms to the function $\mathcal{F}$ defined in eq. (B.23). The result is

$$
Q(s)=\lambda_{T T \mathcal{O}} \frac{3 i \pi^{4}[\Delta(\Delta-4)+20] \sin \left(\frac{\pi \Delta}{2}\right)}{2^{\Delta}(\Delta-6)(\Delta-4) \Delta(\Delta+2) \Gamma\left(\frac{\Delta+4}{2}\right)} s^{(\Delta+2) / 2} .
$$

This can be equated with eq. (B.63) to fix $\tilde{\lambda}_{T T \mathcal{O}}$ in terms of $\lambda_{T T \mathcal{O}}$. Note that $\tilde{\lambda}_{T T \mathcal{O}}$ vanishes when $\Delta=2$, as well as when $\Delta=2 d+2 n$ with $n \in \mathbb{N}$.

The 3 -point function with initial graviton helicities ++ for the energy-momentum tensors is then given by

$$
\left\langle\mathcal{O}\left|\mathrm{T}\left[\tilde{T}^{+}\left(p_{1}\right) \tilde{T}^{+}\left(p_{2}\right)\right]\right| 0\right\rangle=\lambda_{T T \mathcal{O}} \frac{3 i \pi^{4} \sin \left(\frac{\pi}{2} \Delta\right)}{2^{\Delta-4}(\Delta-4)(\Delta-6) \Delta(\Delta+2) \Gamma\left(\frac{\Delta+4}{2}\right)^{2}} s^{\Delta / 2} .
$$

The 3-point function with helicities +- vanishes by angular momentum conservation.

The conformal block is then directly obtained from eq. (B.51):

$$
f(\mathcal{O})=\frac{5}{2} f^{(+)}(\mathcal{O})=\frac{9 \pi^{3} 2^{2 \Delta+2} \sin ^{2}\left(\frac{\pi}{2} \Delta\right)}{(\Delta-6)^{2}(\Delta-4)^{2} \Delta^{4}(\Delta+2)^{4}} \frac{\Gamma\left(\frac{\Delta-1}{2}\right) \Gamma\left(\frac{\Delta+1}{2}\right)}{\Gamma\left(\frac{\Delta+4}{2}\right)^{2}} .
$$

This is the result reported in eq. (3.17). Note that this number grows exponentially fast at asymptotically large scaling dimensions (see figure 2), with the asymptotic form given by

$$
f(\mathcal{O}) \sim 3^{2} 2^{6} \pi^{3} \sin ^{2}\left(\frac{\pi}{2} \Delta\right) \frac{4^{\Delta}}{\Delta^{16}} \quad(\Delta \gg 1) .
$$

Traceless symmetric spin-2 operator. In the case of a traceless, symmetric, spintwo operator $\mathcal{S}^{\mu \nu}$, the relation between the OPE coefficients defined in eq. (B.19) and the quantities $\tilde{\lambda}_{T T \mathcal{S}}^{(1,2)}$ appearing in eq. (B.54) can be worked out in a similar manner from scalar 
contractions. One finds

$$
\begin{aligned}
\left(\begin{array}{c}
\tilde{\lambda}_{T T \mathcal{S}}^{(1)} \\
\tilde{\lambda}_{T T \mathcal{S}}^{(2)}
\end{array}\right)= & \frac{i \pi^{4} \sin \left(\frac{\pi}{2} \Delta\right)}{2^{\Delta-2}(\Delta-6)(\Delta-4) \Delta(\Delta+2) \Gamma\left(\frac{\Delta+6}{2}\right)^{2}} \\
& \times\left(\begin{array}{cc}
\frac{\Delta^{3}+20 \Delta^{2}-8 \Delta-48}{\Delta-8} & -\frac{2 \Delta^{2}+\Delta-4}{2} \\
-\frac{3 \Delta^{3}-30 \Delta^{2}-104 \Delta+96}{\Delta-8} & -\frac{3 \Delta^{2}+6 \Delta-8}{2}
\end{array}\right)\left(\begin{array}{c}
\lambda_{T T \mathcal{S}}^{(1)} \\
\lambda_{T T \mathcal{S}}^{(2)}
\end{array}\right),
\end{aligned}
$$

and therefore

$$
\begin{aligned}
f_{a b}(\mathcal{S})= & \frac{2^{2 \Delta-1} \pi^{3} \sin ^{2}\left(\frac{\pi}{2} \Delta\right)}{(\Delta-6)^{2}(\Delta-4)(\Delta-1) \Delta^{3}(\Delta+2)^{4}(\Delta+4)^{2}} \frac{\Gamma\left(\frac{\Delta-3}{2}\right) \Gamma\left(\frac{\Delta+3}{2}\right)}{\Gamma\left(\frac{\Delta+6}{2}\right)^{2}} \\
& \times\left(\begin{array}{cc}
\frac{9 \Delta^{5}+9 \Delta^{4}+20 \Delta^{3}+1116 \Delta^{2}+4752 \Delta-3456}{(\Delta-8)^{2}} & \frac{18 \Delta^{3}+65 \Delta^{2}+96 \Delta-144}{\Delta-8} \\
-\frac{18 \Delta^{3}+65 \Delta^{2}+96 \Delta-144}{\Delta-8} & \frac{9 \Delta^{3}+27 \Delta^{2}+16 \Delta-48}{8}
\end{array}\right)_{a b}
\end{aligned}
$$

$f_{a b}(\mathcal{S})$ is a positive-definite matrix provided that the unitarity bound $\Delta \geq 4$ is satisfied. Both its eigenvalues vanish when $\Delta=10+2 n$ with $n \in \mathbb{N}$, as well as $\Delta=4$, where the unitarity bound is saturated. A single eigenvalue vanishes when $\Delta=8$. The asymptotic form of the two eigenvalues at large $\Delta$ is

$$
f_{a b}(\mathcal{S}) \sim 3^{2} 2^{6} \pi^{3} \sin ^{2}\left(\frac{\pi}{2} \Delta\right) \frac{4^{\Delta}}{\Delta^{16}}\left(\begin{array}{cc}
\frac{1}{16} & 0 \\
0 & \frac{1}{2}
\end{array}\right)_{a b} \quad(\Delta \gg 1) .
$$

Figure 2 illustrates the behavior of the eigenvalues of $f(\mathcal{S})$ as a function of the scaling dimension $\Delta$.

The analysis can be extended to higher spin operators, at the cost of additional complexity. For instance, with a traceless, symmetric tensor of spin four, there are three tensor structures and as many OPE coefficients. One notable difference with respect to the previous cases is that both polarizations $\mathcal{M}_{++--}$and $\mathcal{M}_{+--+}$can contribute to the conformal blocks. This has been verified in the free scalar theory, in which the spin-four conserved current gives a non-zero contribution to the sum, see eq. (4.10b).

\section{IR finiteness for free theories}

In this appendix, we give some details about the verification of the IR finiteness of the pseudo-amplitude in the free CFTs. As discussed in section 1, it is not sufficient to check the finiteness of the imaginary part of the amplitude. This check is much easier to perform, since it only involves the computation of the total rate $h h \rightarrow \phi \phi, \bar{\psi} \psi, A A$, and we have verified that these are all finite; the results are given in eqs. (4.1a)-(4.3b). To check the finiteness of the real part as well is more delicate, and requires detailed examination of the loop diagrams. Instead of giving the details for all 3 free CFTs, we will take the free scalar theory as demonstrating example. Similar derivations have been made for the case of free fermions and vectors, and we will make some brief remarks about these at the end. 


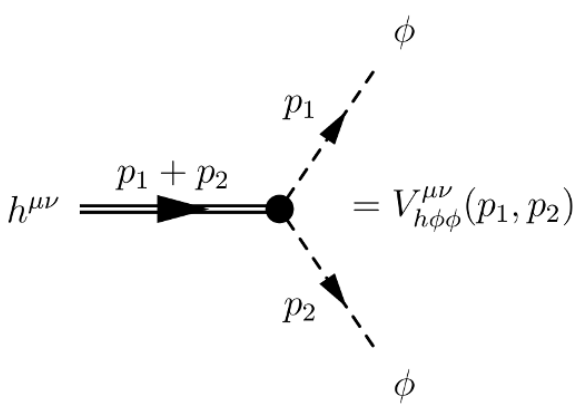

(a)

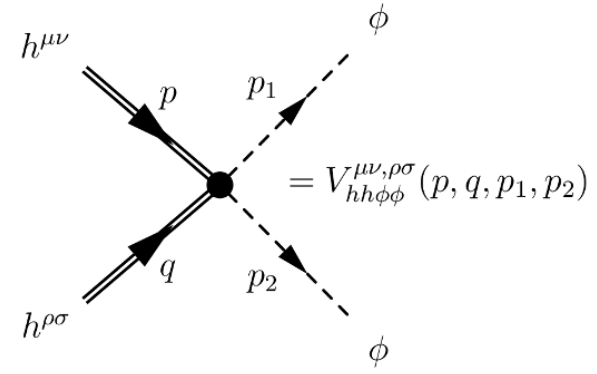

(b)

Figure 6. Feynman rules for the vertices of the free scalar theory.

This appendix is organized as follows. We first give the Feynman rules of the free scalar theory in section C.1. We then show the IR finiteness of the pseudo-amplitude in section C.2. We give an explicit computation of the imaginary part of the pseudo-amplitude in section C.3, and present a check of the normalization of our sum rule for operators near $\Delta=2$ in section C.4

\section{C.1 Feynman rules}

We obtain the Feynman rules by expanding the action in powers of the perturbed graviton field $h_{\mu \nu} \equiv g_{\mu \nu}-\eta_{\mu \nu}$. The interaction vertices we will need are those involving 1 or 2 gravitons. There are diagrams involving a 3-graviton vertex, but we will show that this contribution vanishes identically without requiring the detailed form of the 3 -graviton vertex. The 1- and 2-graviton vertices follow from expanding all quantities to second order in $h_{\mu \nu}$ :

$$
\begin{aligned}
g^{\mu \nu}= & \eta^{\mu \nu}-h^{\mu \nu}+h^{\mu \rho} h_{\rho}^{\nu}, \\
\sqrt{-g}= & 1+\frac{1}{2} h+\frac{1}{8}\left(h^{2}-2 h_{\alpha \beta} h^{\alpha \beta}\right), \\
R= & \left(\partial^{\mu} \partial^{\nu}-\eta^{\mu \nu} \partial^{2}\right) h_{\mu \nu}+h_{\mu \nu}\left(\partial^{\mu} \partial^{\nu} h+\partial^{2} h^{\mu \nu}-2 \partial^{\mu} \partial_{\rho} h^{\nu \rho}\right)-\frac{1}{4} \partial^{\mu} h \partial_{\mu} h \\
& +\frac{3}{4}\left(\partial^{\rho} h^{\mu \nu}\right)\left(\partial_{\rho} h_{\mu \nu}\right)+\left(\partial_{\mu} h \partial_{\nu} h^{\mu \nu}-\frac{1}{2} \partial^{\rho} h^{\mu \nu} \partial_{\mu} h_{\nu \rho}-\left(\partial^{\mu} h_{\mu \rho}\right)\left(\partial_{\nu} h^{\nu \rho}\right),\right.
\end{aligned}
$$

where indices are now raised and lowered using the flat metric. After collecting terms, we find that the 1-graviton vertex is given by (see figure 6)

$$
\begin{aligned}
i V_{h \phi \phi}^{\mu \nu}\left(p_{1}, p_{2}\right)= & \left(p_{1}^{\mu} p_{2}^{\nu}-\frac{1}{2} \eta^{\mu \nu} p_{1} \cdot p_{2}\right) \\
& -\frac{d-2}{4(d-1)}\left[\left(p_{1}+p_{2}\right)^{\mu}\left(p_{1}+p_{2}\right)^{\nu}-\eta^{\mu \nu}\left(p_{1}+p_{2}\right)^{2}\right],
\end{aligned}
$$


while the 2-graviton vertex is given by

$$
\begin{aligned}
V_{h h \phi \phi}^{\mu \nu, \rho \sigma}\left(p, q, p_{1}, p_{2}\right) \\
=i\left\{\begin{array}{l}
-\frac{1}{2} \eta^{\rho \sigma} p_{1}^{\mu} p_{2}^{\nu}-\frac{1}{2} \eta^{\mu \nu} p_{1}^{\rho} p_{2}^{\sigma}+\eta^{\nu \sigma}\left(p_{1}^{\mu} p_{2}^{\rho}+p_{1}^{\rho} p_{2}^{\mu}\right)+\frac{1}{4}\left(\eta^{\mu \nu} \eta^{\rho \sigma}-2 \eta^{\mu \rho} \eta^{\nu \sigma}\right)\left(p_{1} \cdot p_{2}\right) \\
+\frac{d-2}{4(d-1)}\left[\begin{array}{l}
\eta^{\rho \sigma}\left(q^{\mu} q^{\nu}+\frac{1}{2} p^{\mu} p^{\nu}+p^{\mu} q^{\nu}\right)+\eta^{\mu \nu}\left(p^{\rho} p^{\sigma}+\frac{1}{2} q^{\rho} q^{\sigma}+q^{\rho} p^{\sigma}\right) \\
-2 \eta^{\nu \sigma}\left(p^{\mu} p^{\rho}+q^{\mu} q^{\rho}+p^{\mu} q^{\rho}+\frac{1}{2} q^{\mu} p^{\rho}\right) \\
-\frac{1}{2}\left(\eta^{\mu \nu} \eta^{\rho \sigma}-3 \eta^{\mu \rho} \eta^{\nu \sigma}\right)(p \cdot q)-\frac{1}{2}\left(\eta^{\mu \nu} \eta^{\rho \sigma}-2 \eta^{\mu \rho} \eta^{\nu \sigma}\right)\left(p^{2}+q^{2}\right)
\end{array}\right]
\end{array}\right\} .
\end{aligned}
$$

\section{C.2 IR finiteness of the pseudo-amplitude}

The pseudo-amplitude is given by the 1-loop Feynman diagrams shown in figure 7 . To examine the IR finiteness, we use the method of "expansion by regions" [18]. This can be used to obtain the asymptotic behavior of a Feynman diagram in various kinematic limits by summing over the contributions from relevant regions of the loop momenta that contribute to the dimensionally regularized integral. To establish IR finiteness of the diagram it is sufficient to examine all the dangerous (potentially IR divergent) regions and establish that they are finite. This amounts to a power-counting argument, and is simpler and more transparent than the direct evaluation of diagrams, where the IR finiteness appears to result from miraculous cancellations. For 1-loop diagrams, the only dangerous regions are known to be the soft, ultra-soft, and collinear limits. We will consider first the massless limit $-p_{i}^{2}=m^{2} \rightarrow 0$ with $s, t$ fixed, and then the limit $t \rightarrow 0$ with $m^{2}=0$ and $s$ fixed.

We will discuss the box diagram in figure 7 (a) in detail, and make only brief remarks about the other diagrams. Before taking either the massless or the forward limit, the box diagram contributes

$$
i \mathcal{M} \propto\left\{\begin{array}{l}
\int \frac{d^{4} k}{(2 \pi)^{4}} \frac{1}{k^{2}\left(k+p_{1}\right)^{2}\left(k-p_{2}\right)^{2}\left(k+p_{1}+p_{3}\right)^{2}} \epsilon_{\mu_{1} \nu_{1}}\left(p_{1}\right) \epsilon_{\mu_{2} \nu_{2}}\left(p_{2}\right) \epsilon_{\mu_{3} \nu_{3}}\left(p_{3}\right) \epsilon_{\mu_{4} \nu_{4}}\left(p_{4}\right) \\
\times\left[k^{\mu_{1}}\left(k+p_{1}\right)^{\nu_{1}}-\frac{1}{2} \eta^{\mu_{1} \nu_{1}} k \cdot\left(k+p_{1}\right)-\frac{1}{6} \eta^{\mu_{1} \nu_{1}} m^{2}\right] \\
\times\left[k^{\mu_{2}}\left(k-p_{2}\right)^{\nu_{2}}-\frac{1}{2} \eta^{\mu_{2} \nu_{2}} k \cdot\left(k-p_{2}\right)-\frac{1}{6} \eta^{\mu_{2} \nu_{2}} m^{2}\right] \\
\times\left[\left(k+p_{1}\right)^{\mu_{3}}\left(k+p_{1}+p_{3}\right)^{\nu_{3}}-\frac{1}{2} \eta^{\mu_{3} \nu_{3}}\left(k+p_{1}\right) \cdot\left(k+p_{1}+p_{3}\right)-\frac{1}{6} \eta^{\mu_{3} \nu_{3}} m^{2}\right] \\
\times\left[\left(k-p_{2}\right)^{\mu_{4}}\left(k+p_{1}+p_{3}\right)^{\nu_{4}}-\frac{1}{2} \eta^{\mu_{4} \nu_{4}}\left(k-p_{2}\right) \cdot\left(k+p_{1}+p_{3}\right)-\frac{1}{6} \eta^{\mu_{4} \nu_{4}} m^{2}\right]
\end{array}\right\} .
$$

Massless limit. We begin with the massless limit $m^{2} \ll s, t \sim Q^{2}$. The soft region is defined as the regime in which all the components of $k$ are of order $m$, i.e. $k^{\mu} \sim m$. The ultra-soft region is defined by $k^{\mu} \sim \frac{m^{2}}{Q}$. In these two regions, the propagator part of the loop integral can be reduced to

$$
\frac{d^{4} k}{k^{2}\left(k+p_{1}\right)^{2}\left(k-p_{2}\right)^{2}\left(k+p_{1}+p_{3}\right)^{2}} \sim \frac{d^{4} k}{k^{2}(k \cdot Q)(k \cdot Q) Q^{2}},
$$




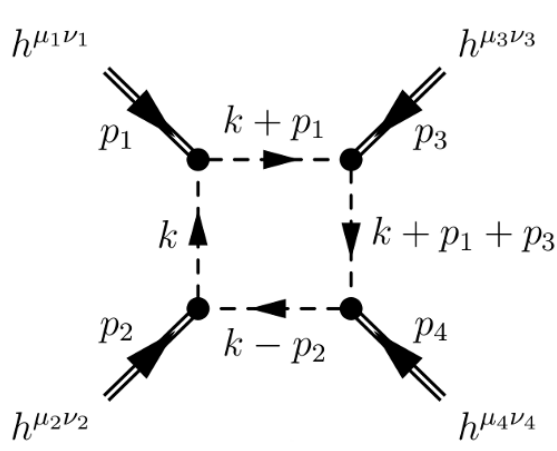

(a) Box

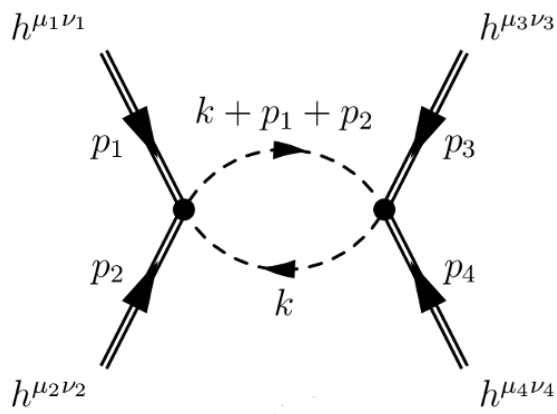

(c) Bubble

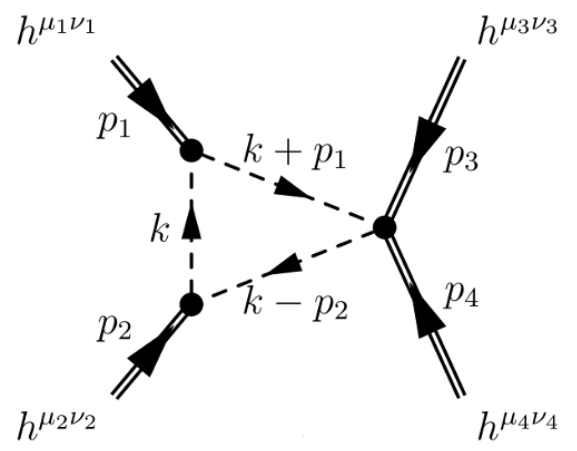

(b) Triangle

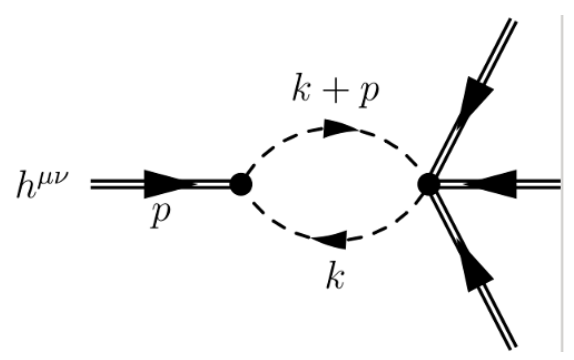

(d) 3-graviton

Figure 7. Feynman diagrams for the pseudo-amplitude in the free scalar theory.

which is at most logarithmically IR divergent. Both vertices associated with $p_{1}$ and $p_{2}$ give a further suppression of at least one power of $k \sim m$,

$$
\begin{aligned}
& {\left[k^{\mu_{1}}\left(k+p_{1}\right)^{\nu_{1}}-\frac{1}{2} \eta^{\mu_{1} \nu_{1}} k \cdot\left(k+p_{1}\right)-\frac{1}{6} \eta^{\mu_{1} \nu_{1}} m^{2}\right] \lesssim O(k),} \\
& {\left[k^{\mu_{2}}\left(k-p_{2}\right)^{\nu_{2}}-\frac{1}{2} \eta^{\mu_{2} \nu_{2}} k \cdot\left(k-p_{2}\right)-\frac{1}{6} \eta^{\mu_{2} \nu_{2}} m^{2}\right] \lesssim O(k) .}
\end{aligned}
$$

Therefore, contributions from the soft and ultra-soft regions to the box diagram are finite.

The collinear regions are slightly more complicated. They correspond to the case where the internal momentum at a vertex becomes collinear with the external momentum. For example, the collinear region for the vertex associated with $p_{1}^{\mu}=\frac{\sqrt{s}}{2}\left(1,0,0, \sqrt{1-4 m^{2} / s}\right)$ is defined as

$$
\left\{\begin{array}{l}
k_{+} \equiv k^{0}+k^{3} \sim Q \\
k_{-} \equiv k^{0}-k^{3} \sim \frac{m^{2}}{Q} \\
k_{\perp}^{i} \equiv\left(k^{1}, k^{2}\right) \sim m
\end{array},\right.
$$

which gives $k^{2} \sim m^{2}, p_{1} \cdot k \sim m^{2}$, and $k^{\mu} \propto p_{1}^{\mu}+O(m)$. Other collinear regions are defined similarly, and can be obtained by permutations of momenta. In the collinear region defined by eq. (C.7), the propagator part goes as

$$
\frac{d^{4} k}{k^{2}\left(k+p_{1}\right)^{2}\left(k-p_{2}\right)^{2}\left(k+p_{1}+p_{3}\right)^{2}} \sim \frac{m^{4}}{m^{2} m^{2} Q^{2} Q^{2}} .
$$


This is again logarithmic divergent by itself, but the vertex associated with $p_{1}$ contributes an additional suppression factor

$$
\left[k^{\mu_{1}}\left(k+p_{1}\right)^{\nu_{1}}-\frac{1}{2} \eta^{\mu_{1} \nu_{1}} k \cdot\left(k+p_{1}\right)-\frac{1}{6} \eta^{\mu_{1} \nu_{1}} m^{2}\right] \lesssim O\left(m^{2}\right) .
$$

Here we have used $p_{1} \cdot k \sim m^{2}$ as well as the transversality of the graviton polarization tensor $p_{1}^{\mu_{1}} \epsilon_{\mu_{1} \nu_{1}}\left(p_{1}\right)=0$. We see that the contribution from the collinear region is also finite.

Forward limit. We now check the forward scattering limit $t \ll s \sim Q^{2}$, setting $m^{2}=0$. In this case, the pseudo-amplitude in eq. (C.4) becomes

$$
i \mathcal{M} \propto\left\{\begin{array}{l}
\int \frac{d^{4} k}{(2 \pi)^{4}} \frac{1}{k^{2}\left(k+p_{1}\right)^{2}\left(k-p_{2}\right)^{2}\left(k+p_{1}+p_{3}\right)^{2}} \epsilon_{\mu_{1} \nu_{1}}\left(p_{1}\right) \epsilon_{\mu_{2} \nu_{2}}\left(p_{2}\right) \epsilon_{\mu_{3} \nu_{3}}\left(p_{3}\right) \epsilon_{\mu_{4} \nu_{4}}\left(p_{4}\right) \\
\times\left[k^{\mu_{1}}\left(k+p_{1}\right)^{\nu_{1}}-\frac{1}{2} \eta^{\mu_{1} \nu_{1}} k \cdot\left(k+p_{1}\right)\right]\left[k^{\mu_{2}}\left(k-p_{2}\right)^{\nu_{2}}-\frac{1}{2} \eta^{\mu_{2} \nu_{2}} k \cdot\left(k-p_{2}\right)\right] \\
\times\left[\left(k+p_{1}\right)^{\mu_{3}}\left(k+p_{1}+p_{3}\right)^{\nu_{3}}-\frac{1}{2} \eta^{\mu_{3} \nu_{3}}\left(k+p_{1}\right) \cdot\left(k+p_{1}+p_{3}\right)\right] \\
\times\left[\left(k-p_{2}\right)^{\mu_{4}}\left(k+p_{1}+p_{3}\right)^{\nu_{4}}-\frac{1}{2} \eta^{\mu_{4} \nu_{4}}\left(k-p_{2}\right) \cdot\left(k+p_{1}+p_{3}\right)\right]
\end{array}\right\}
$$

In the soft region $k^{\mu} \sim \sqrt{t}$ and the ultra-soft region $k^{\mu} \sim t / Q^{2}$, the propagator part goes like

$$
\frac{d^{4} k}{k^{2}\left(k+p_{1}\right)^{2}\left(k-p_{2}\right)^{2}\left(k+p_{1}+p_{3}\right)^{2}} \sim \frac{d^{4} k}{k^{2}(k \cdot Q)(k \cdot Q) t},
$$

which has a $1 / t$ divergence. However, for transverse and traceless graviton polarizations, both vertices associated with $p_{1}$ and $p_{2}$ give a suppression factor of at least two powers of $k$ :

$$
\begin{aligned}
& {\left[k^{\mu_{1}}\left(k+p_{1}\right)^{\nu_{1}}-\frac{1}{2} \eta^{\mu_{1} \nu_{1}} k \cdot\left(k+p_{1}\right)\right] \rightarrow k^{\mu_{1}} k^{\nu_{1}} \lesssim O\left(k^{2}\right),} \\
& {\left[k^{\mu_{2}}\left(k-p_{2}\right)^{\nu_{2}}-\frac{1}{2} \eta^{\mu_{2} \nu_{2}} k \cdot\left(k-p_{2}\right)\right] \rightarrow k^{\mu_{2}} k^{\nu_{2}} \lesssim O\left(k^{2}\right) .}
\end{aligned}
$$

Together, they make the contribution from these regions IR finite.

In the $p_{1}$ collinear region defined by eq. (C.7) (with $m$ replaced by $\sqrt{t}$ ), we have $k \propto p_{1}+O(\sqrt{t}) \propto p_{3}+O(\sqrt{t})$. The propagator part is $1 / t$ IR divergent

$$
\frac{d^{4} k}{k^{2}\left(k+p_{1}\right)^{2}\left(k-p_{2}\right)^{2}\left(k+p_{1}+p_{3}\right)^{2}} \sim \frac{t^{2}}{t \cdot t \cdot Q^{2} \cdot t},
$$

but both the $p_{1}$ and $p_{3}$ vertices give a suppression factor proportional to $t$ :

$$
\begin{aligned}
{\left[k^{\mu_{1}}\left(k+p_{1}\right)^{\nu_{1}}-\frac{1}{2} \eta^{\mu_{1} \nu_{1}} k \cdot\left(k+p_{1}\right)\right] } & \lesssim O(t), \\
{\left[\left(k+p_{1}\right)^{\mu_{3}}\left(k+p_{1}+p_{3}\right)^{\nu_{3}}-\frac{1}{2} \eta^{\mu_{3} \nu_{3}}\left(k+p_{1}\right) \cdot\left(k+p_{1}+p_{3}\right)\right] } & \lesssim O(t),
\end{aligned}
$$

where we have again used the transversality of the gravitons polarizations. 
Summary. We have shown that the box diagram in figure $7(\mathrm{a})$ is IR finite under both the massless limit and the forward limit, assuming transverse and traceless polarizations for the gravitons. One can repeat the above procedure for the triangle diagram in figure $7(\mathrm{~b})$ and the bubble diagram in figure $7(\mathrm{c})$. They also turn out to be IR finite under both limits using similar reasoning.

In addition, there is a special kind of diagram with the 3 -graviton vertex shown in figure $7(\mathrm{~d})$. This diagram depends on a single external momentum $p=p_{i}$. We hence expect it to vanish under the massless limit $-p_{i}^{2}=m^{2} \rightarrow 0$. However, before we can claim this, the IR finiteness under this limit has to be checked. This actually can be shown regardless of the details of the 3-graviton vertex. The diagram has the form:

$$
i \mathcal{M} \propto \int \frac{d^{4} k}{(2 \pi)^{4}} \frac{1}{k^{2}(k+p)^{2}} \epsilon_{\mu \nu}(p) V_{h \phi \phi}^{\mu \nu}(k+p,-k) V_{3},
$$

where we have denoted the the 3 -graviton vertex by $V_{3}$. Because $V_{3}$ is a polynomial of $p, k$ contracted with the three external polarization tensors, it is finite in the limit $m^{2} \rightarrow 0$, and we only need to check for IR divergences in the rest of the diagram. The only dangerous region for eq. (C.15) is the collinear region defined in eq. (C.7). The propagator part is logrithmically divergent in this region

$$
\frac{d^{4} k}{k^{2}(k+p)^{2}} \sim \frac{m^{4}}{m^{2} m^{2}}
$$

But the 1-graviton vertex provides additional suppression factors

$$
\epsilon_{\mu \nu}(p) V_{h \phi \phi}^{\mu \nu}(k+p,-k) \lesssim O(m)
$$

since $k^{\mu} \propto p^{\mu}+O(m)$ in the collinear region.

For free theories of fermions and vectors, we have checked that IR finiteness holds by a similar analysis. For fermions, we must consider perturbations of the vierbein field $\varphi^{a}{ }_{\mu} \equiv e^{a}{ }_{\mu}-\delta^{a}{ }_{\mu}$, which corresponds to a metric perturbation $h_{\mu \nu}=\varphi_{\mu \nu}+\varphi_{\nu \mu}+\eta_{a b} \varphi^{a}{ }_{\mu} \varphi^{b}{ }_{\nu}$. We express the vierbein in terms of the metric perturbation by making the gauge choice $\varphi_{\mu \nu}=\varphi_{\nu \mu}$ to define the energy-momentum tensor. In the free vector case, we follow the usual gauge-fixing procedure, including ghost fields to subtract the unphysical vector intermediate states in the TTTT correlator.

\section{C.3 Imaginary part of the pseudo-amplitude}

In free CFTs, the full imaginary part of the pseudo-amplitude in the forward limit $\operatorname{Im} \mathcal{M}_{T_{1} T_{2} \rightarrow T_{3} T_{4}}(s) \propto \sigma\left(h_{1} h_{2} \rightarrow \mathrm{CFT}\right)$ can be directly evaluated, due to the asymptotic particle interpretation of the CFT states. This calculation is detailed below for the free scalar theory. In this case, the concrete form of the above relation is

$$
2 \operatorname{Im} \mathcal{M}_{T_{i} T_{j} \rightarrow T_{i} T_{j}}(s)=(2 i)^{4} \int d \Pi_{\phi \phi}\left(k_{1}, k_{2}\right)\left|\mathcal{M}_{h_{i} h_{j} \rightarrow \phi \phi}\left(k_{1}, k_{2}\right)\right|^{2},
$$

where the two-body phase space can be written in terms of the scattering angles as

$$
\int d \Pi_{\phi \phi}\left(k_{1}, k_{2}\right)=\frac{1}{2} \frac{1}{8 \pi} \int_{-1}^{1} \frac{d(\cos \theta)}{2} .
$$




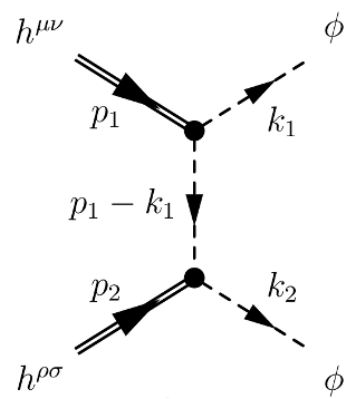

(a)

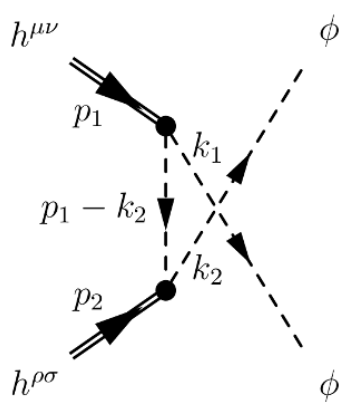

(b)

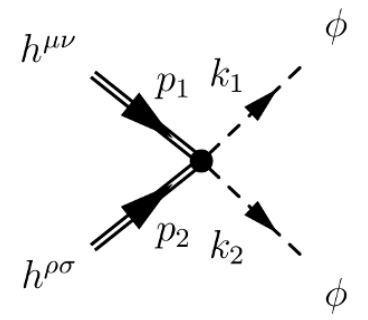

(c)

Figure 8. Tree-level diagrams relevant to the $h h \rightarrow \phi \phi$ scattering process in the free scalar theory.

Note the presence of the symmetry factor $1 / 2$ due to identical final-state particles. There are three diagrams for the scattering amplitude $\mathcal{M}_{h_{1} h_{2} \rightarrow \phi \phi}\left(k_{1}, k_{2}\right)$, as shown in figure 8 . They add up to the total amplitude

$$
\mathcal{M}_{h_{1} h_{2} \rightarrow \phi \phi}=\epsilon_{\mu \nu}^{1}\left(p_{1}\right) \epsilon_{\rho \sigma}^{2}\left(p_{2}\right)\left\{\begin{array}{c}
\frac{k_{1}^{\mu} k_{1}^{\nu} k_{2}^{\rho} k_{2}^{\sigma}}{\left(p_{1}-k_{1}\right)^{2}}+\frac{k_{2}^{\mu} k_{2}^{\nu} k_{1}^{\rho} k_{1}^{\sigma}}{\left(p_{1}-k_{2}\right)^{2}} \\
+\eta^{\nu \sigma}\left[-\frac{1}{2} \eta^{\mu \rho}\left(k_{1} \cdot k_{2}\right)+\left(k_{1}^{\mu} k_{2}^{\rho}+k_{2}^{\mu} k_{1}^{\rho}\right)\right. \\
\left.-\frac{1}{6}\left(p_{2}^{\mu} p_{1}^{\rho}-\frac{3}{2} \eta^{\mu \rho} p_{1} \cdot p_{2}\right)\right]
\end{array}\right\} .
$$

Here, we have readily taken the massless limit $-p_{1}^{2}=-p_{2}^{2}=m^{2} \rightarrow 0$. Splitting the result into the two helicity structures (same or opposite), and expressing it in terms of the scattering angle $\theta$ and the azimuthal angle $\varphi$, we get

$$
\begin{aligned}
\mathcal{M}_{h^{+} h^{+} \rightarrow \phi \phi}(\theta, \varphi) & =\epsilon_{\mu \nu}^{+}\left(p_{1}\right) \epsilon_{\rho \sigma}^{+}\left(p_{2}\right)\left[\frac{k_{1}^{\mu} k_{1}^{\nu} k_{2}^{\rho} k_{2}^{\sigma}}{\left(p_{1}-k_{1}\right)^{2}}+\frac{k_{2}^{\mu} k_{2}^{\nu} k_{1}^{\rho} k_{1}^{\sigma}}{\left(p_{1}-k_{2}\right)^{2}}+\eta^{\nu \sigma}\left(\frac{s}{8} \eta^{\mu \rho}+2 k_{1}^{\mu} k_{2}^{\rho}\right)\right] \\
& =\frac{s}{16}\left(3 \cos ^{2} \theta-1\right), \\
\mathcal{M}_{h^{+} h^{-} \rightarrow \phi \phi}(\theta, \varphi) & =\epsilon_{\mu \nu}^{+}\left(p_{1}\right) \epsilon_{\rho \sigma}^{-}\left(p_{2}\right)\left[\frac{k_{1}^{\mu} k_{1}^{\nu} k_{2}^{\rho} k_{2}^{\sigma}}{\left(p_{1}-k_{1}\right)^{2}}+\frac{k_{2}^{\mu} k_{2}^{\nu} k_{1}^{\rho} k_{1}^{\sigma}}{\left(p_{1}-k_{2}\right)^{2}}\right] \\
& =\frac{s}{16} e^{4 i \varphi}\left(1-\cos ^{2} \theta\right) .
\end{aligned}
$$

Making use of eqs. (C.18) and (C.19), we get the finite results

$$
\begin{aligned}
& 2 \operatorname{Im} \mathcal{M}_{++}^{\text {scalar }}(s)=\frac{1}{320 \pi} s^{2}, \\
& 2 \operatorname{Im} \mathcal{M}_{+-}^{\text {scalar }}(s)=\frac{1}{480 \pi} s^{2} .
\end{aligned}
$$

The imaginary part of the pseudo-amplitude can be calculated in the same way in free fermion and vector theories.

\section{C.4 $\phi^{2}$ contribution in general $d$}

We now show how to use the contribution of the $\phi^{2}$ operator in free scalar theory away from $d=4$ to check the normalization of the coefficients appearing in our sum rule. Although 
the sum rule is only valid for $d=4$, the optical theorem eq. (2.6) is valid for any $d$, and the contribution of $\phi^{2}$ to the imaginary part of the amplitude is given by ${ }^{18}$

$$
\operatorname{Im} \mathcal{M}_{++--}(s, d)=5 \pi s^{d / 2} \lambda_{T T \phi^{2}}^{2} f^{(+)}\left(\phi^{2}, d\right)+\cdots .
$$

Defining this requires the analytic continuation of the polarization tensors to general $d$, but in practice the contribution of $\phi^{2}$ can be computed using only the $d$-independent relations

$$
p_{i \mu} \epsilon_{ \pm}^{\mu \nu}\left(p_{i}\right)=0, \quad \eta_{\mu \nu} \epsilon_{ \pm}^{\mu \nu}\left(p_{i}\right)=0, \quad \eta_{\mu \rho} \eta_{\nu \sigma} \epsilon_{+}^{\mu \nu}\left(p_{1}\right) \epsilon_{+}^{\rho \sigma}\left(p_{2}\right)=1
$$

The calculation in the free scalar theory then gives

$$
5 \pi \lambda_{T T \phi^{2}}^{2} f^{(+)}\left(\phi^{2}, d\right)=\frac{(d-2)^{2}(d-4)^{2}}{2^{2 d+3} \pi^{(d-3) / 2} d^{2}(d-1) \Gamma\left(\frac{d+1}{2}\right)} .
$$

This can be thought of as the contribution of an operator of dimension $\Delta=d-2$ in general $d$. It has a double zero as $d \rightarrow 4$, consistent with the fact that there is a double zero in the contribution of a scalar operator $\mathcal{O}$ with dimension $\Delta$ in $d=4$ in the limit $\Delta \rightarrow 2$. In fact, if we assume that the contribution of a general scalar operator of dimension $\Delta$ in $d$ spacetime dimensions is an analytic function of $\Delta$ and $d$, then the coefficient of the double zero at the point $d=4, \Delta=2$ must agree. Using our convention eq. (B.14), the OPE coefficient in the free scalar theory is given by

$$
\lambda_{T T \phi^{2}}=\frac{d(d-2)^{2}}{2 \sqrt{2}(d-1)} C_{T} \stackrel{d \rightarrow 4}{\longrightarrow} \frac{4 \sqrt{2}}{9 \pi^{4}},
$$

which gives

$$
\frac{5}{2} f^{(+)}\left(\phi^{2}, d\right) \stackrel{d \rightarrow 4}{\longrightarrow} \frac{3^{2} \pi^{6}}{2^{17}}(\Delta-2)^{2},
$$

where $\Delta=d-2$. This precisely agrees with eq. (3.17), giving an independent check of the normalization of $f(\mathcal{O})$.

Open Access. This article is distributed under the terms of the Creative Commons Attribution License (CC-BY 4.0), which permits any use, distribution and reproduction in any medium, provided the original author(s) and source are credited.

\section{References}

[1] H. Georgi, Unparticle physics, Phys. Rev. Lett. 98 (2007) 221601 [hep-ph/0703260] [INSPIRE].

[2] H. Georgi, Another odd thing about unparticle physics, Phys. Lett. B 650 (2007) 275 [arXiv: 0704.2457] [INSPIRE].

[3] M. Gillioz, X. Lu and M.A. Luty, Scale Anomalies, States and Rates in Conformal Field Theory, JHEP 04 (2017) 171 [arXiv: 1612.07800] [InSPIRE].

\footnotetext{
${ }^{18}$ The operator that satisfies the normalization eq. (B.2) is really $\frac{1}{\sqrt{2}} \phi^{2}$.
} 
[4] J. Gomis, P.-S. Hsin, Z. Komargodski, A. Schwimmer, N. Seiberg and S. Theisen, Anomalies, Conformal Manifolds and Spheres, JHEP 03 (2016) 022 [arXiv: 1509.08511] [INSPIRE].

[5] Y. Nakayama, Can we change $c$ in four-dimensional CFTs by exactly marginal deformations?, JHEP 07 (2017) 004 [arXiv: 1702.02324] [INSPIRE].

[6] Z. Komargodski and A. Schwimmer, On Renormalization Group Flows in Four Dimensions, JHEP 12 (2011) 099 [arXiv: 1107.3987] [INSPIRE].

[7] H. Casini, E. Testé and G. Torroba, Markov Property of the Conformal Field Theory Vacuum and the a Theorem, Phys. Rev. Lett. 118 (2017) 261602 [arXiv:1704.01870] [INSPIRE].

[8] M.A. Luty, J. Polchinski and R. Rattazzi, The a-theorem and the Asymptotics of $4 D$ Quantum Field Theory, JHEP 01 (2013) 152 [arXiv:1204.5221] [INSPIRE].

[9] A. Dymarsky, Z. Komargodski, A. Schwimmer and S. Theisen, On Scale and Conformal Invariance in Four Dimensions, JHEP 10 (2015) 171 [arXiv: 1309.2921] [INSPIRE].

[10] C. Cordova, J. Maldacena and G.J. Turiaci, Bounds on OPE Coefficients from Interference Effects in the Conformal Collider, JHEP 11 (2017) 032 [arXiv:1710.03199] [INSPIRE].

[11] T. Faulkner, R.G. Leigh, O. Parrikar and H. Wang, Modular Hamiltonians for Deformed Half-Spaces and the Averaged Null Energy Condition, JHEP 09 (2016) 038 [arXiv: 1605.08072] [INSPIRE].

[12] T. Hartman, S. Kundu and A. Tajdini, Averaged Null Energy Condition from Causality, JHEP 07 (2017) 066 [arXiv: 1610.05308] [INSPIRE].

[13] D. Meltzer and E. Perlmutter, Beyond $a=c$ : gravitational couplings to matter and the stress tensor OPE, JHEP 07 (2018) 157 [arXiv: 1712.04861] [INSPIRE].

[14] E. Katz, Z.U. Khandker and M.T. Walters, A Conformal Truncation Framework for Infinite-Volume Dynamics, JHEP 07 (2016) 140 [arXiv:1604.01766] [INSPIRE].

[15] S. Weinberg, The Quantum theory of fields. Vol. 1: Foundations, Cambridge University Press, Cambridge U.K.(2005).

[16] D. Pappadopulo, S. Rychkov, J. Espin and R. Rattazzi, OPE Convergence in Conformal Field Theory, Phys. Rev. D 86 (2012) 105043 [arXiv: 1208.6449] [INSPIRE].

[17] S. Rychkov and P. Yvernay, Remarks on the Convergence Properties of the Conformal Block Expansion, Phys. Lett. B 753 (2016) 682 [arXiv:1510.08486] [INSPIRE].

[18] V.A. Smirnov, Applied asymptotic expansions in momenta and masses, Springer Tracts Mod. Phys. 177 (2002) 1.

[19] R.M. Wald, General Relativity, Chicago University Press, Chicago U.S.A. (1984).

[20] M.J. Duff, Observations on Conformal Anomalies, Nucl. Phys. B 125 (1977) 334 [InSPIRE].

[21] H. Osborn and A.C. Petkou, Implications of conformal invariance in field theories for general dimensions, Annals Phys. 231 (1994) 311 [hep-th/9307010] [INSPIRE].

[22] M.S. Costa, J. Penedones, D. Poland and S. Rychkov, Spinning Conformal Correlators, JHEP 11 (2011) 071 [arXiv: 1107.3554] [INSPIRE].

[23] A. Dymarsky, On the four-point function of the stress-energy tensors in a CFT, JHEP 10 (2015) 075 [arXiv: 1311.4546] [INSPIRE].

[24] J.M. Maldacena and G.L. Pimentel, On graviton non-Gaussianities during inflation, JHEP 09 (2011) 045 [arXiv: 1104.2846] [INSPIRE]. 
[25] C. Corianò, M.M. Maglio and E. Mottola, TTT in CFT: Trace Identities and the Conformal Anomaly Effective Action, arXiv:1703.08860 [INSPIRE].

[26] A. Bzowski, P. McFadden and K. Skenderis, Implications of conformal invariance in momentum space, JHEP 03 (2014) 111 [arXiv: 1304.7760] [INSPIRE].

[27] A. Bzowski, P. McFadden and K. Skenderis, Renormalised 3-point functions of stress tensors and conserved currents in CFT, arXiv:1711.09105 [INSPIRE].

[28] V.K. Dobrev, V.B. Petkova, S.G. Petrova and I.T. Todorov, Dynamical Derivation of Vacuum Operator Product Expansion in Euclidean Conformal Quantum Field Theory, Phys. Rev. D 13 (1976) 887 [INSPIRE].

[29] A. Dymarsky, F. Kos, P. Kravchuk, D. Poland and D. Simmons-Duffin, The 3d Stress-Tensor Bootstrap, JHEP 02 (2018) 164 [arXiv:1708.05718] [INSPIRE].

[30] S. Ferrara, A.F. Grillo, G. Parisi and R. Gatto, The shadow operator formalism for conformal algebra. vacuum expectation values and operator products, Lett. Nuovo Cim. 4S2 (1972) 115 [INSPIRE].

[31] D. Simmons-Duffin, Projectors, Shadows and Conformal Blocks, JHEP 04 (2014) 146 [arXiv: 1204.3894] [INSPIRE]. 